\title{
Novel molecular imaging methods for cancer detection
}

Citation for published version (APA):

Panth, K. M. (2016). Novel molecular imaging methods for cancer detection: more than meets the eye . [Doctoral Thesis, Maastricht University]. Datawyse / Universitaire Pers Maastricht. https://doi.org/10.26481/dis.20160630kp

Document status and date:

Published: 01/01/2016

DOI:

10.26481/dis.20160630kp

Document Version:

Publisher's PDF, also known as Version of record

\section{Please check the document version of this publication:}

- A submitted manuscript is the version of the article upon submission and before peer-review. There can be important differences between the submitted version and the official published version of record.

People interested in the research are advised to contact the author for the final version of the publication, or visit the DOI to the publisher's website.

- The final author version and the galley proof are versions of the publication after peer review.

- The final published version features the final layout of the paper including the volume, issue and page numbers.

Link to publication

\footnotetext{
General rights rights.

- You may freely distribute the URL identifying the publication in the public portal. please follow below link for the End User Agreement:

www.umlib.nl/taverne-license

Take down policy

If you believe that this document breaches copyright please contact us at:

repository@maastrichtuniversity.nl

providing details and we will investigate your claim.
}

Copyright and moral rights for the publications made accessible in the public portal are retained by the authors and/or other copyright owners and it is a condition of accessing publications that users recognise and abide by the legal requirements associated with these

- Users may download and print one copy of any publication from the public portal for the purpose of private study or research.

- You may not further distribute the material or use it for any profit-making activity or commercial gain

If the publication is distributed under the terms of Article $25 \mathrm{fa}$ of the Dutch Copyright Act, indicated by the "Taverne" license above, 


\section{Novel Molecular Imaging Methods for Cancer Detection: More than Meets the Eye}

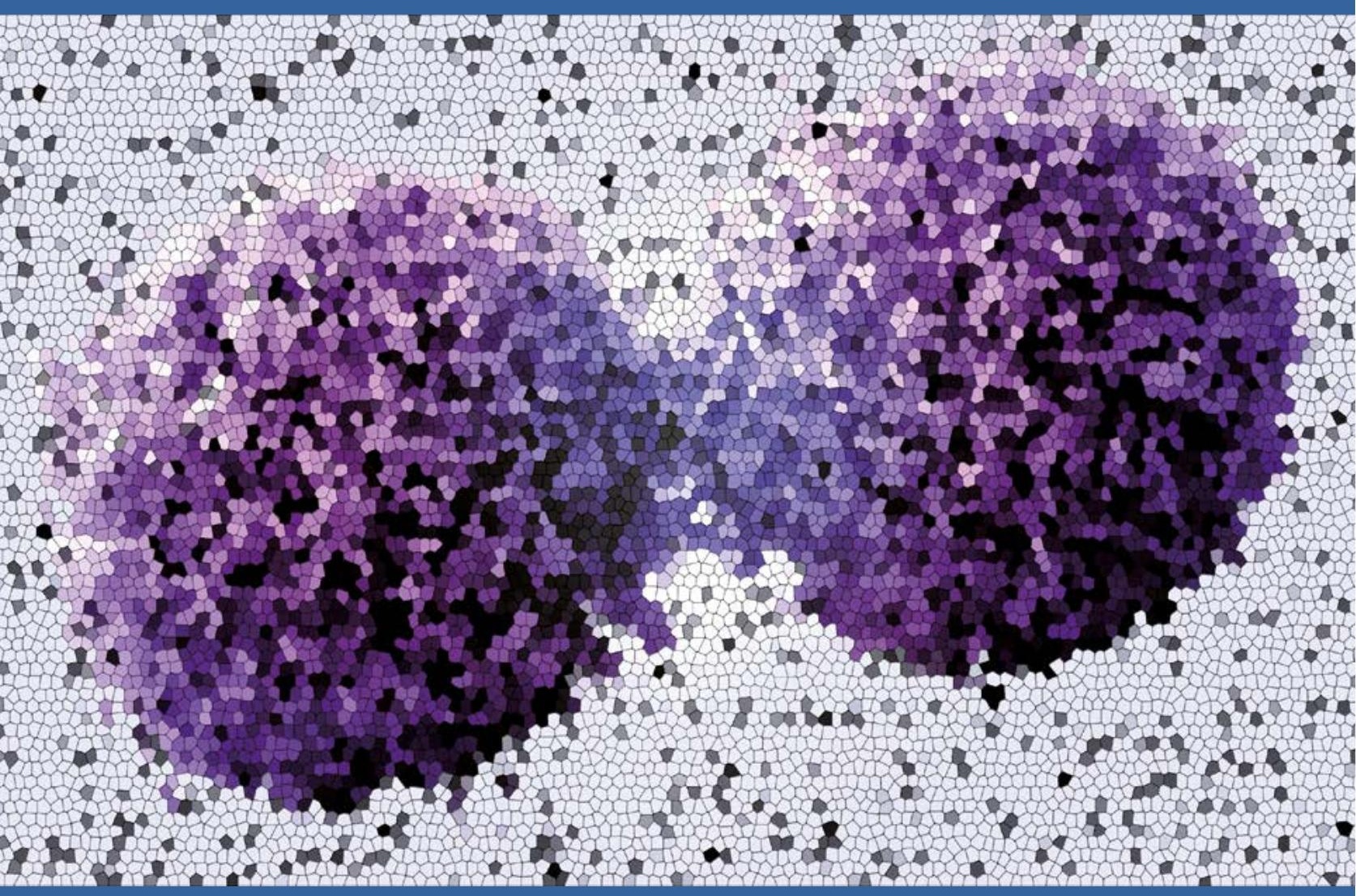


Cover:

Vaguely visible molecular information in two dividing cancer cells unveiled by imaging

Cover design: Prof. Dr. Navin Kumar C. Twarakavi

Layout: Kranthi Marella Panth

Production:

Printing: Datawyse | Universitaire Pers Maastricht

ISBN: 9789461595782

C Kranthi Marella Panth, Maastricht, 2016 


\section{Novel molecular imaging methods for cancer detection:}

\section{More than meets the eye}

to obtain a doctoral degree at Maastricht University, on the authority of the Rector Magnificus, Prof. dr. L.L.G. Soete, in accordance with the decision of the Board of Deans, to be defended in public on

Thursday, June $30^{\text {th }}, 2016$ at $10: 00$ hours

by

Kranthi Marella Panth 


\section{Promoter:}

Prof. dr. P. Lambin

\section{Co-promoter:}

Dr. L.J Dubois

Dr. A. Yaromina

\section{Assessment committee:}

Prof. Dr. F.C.S Ramaekers (chairman)

Prof. Dr. Dario Neri, ETH Zurich

Dr. Hugo Aerts, Dana Farber Cancer Institute, Boston

Prof. Dr. F. Mottaghy

Prof. Dr. Andre Dekker 
Chapter 1 Introduction and outline

Chapter 2 New ways to image and target tumor hypoxia and its molecular responses

Part 1

Evaluation of new antibody based imaging tracers for detecting invasive phenotype

Chapter 3 PET imaging with the $\mathrm{Zr}^{89}$ labeled EGFR antibody, cetuximab

Chapter 4 Optical imaging of Matrix Metallo Proteinase 2 (MMP2)

Part 2

CT derived radiomics and underlying tumor heterogeneities: proofof-concept studies $\begin{array}{ll}\text { Chapter } 5 & \text { Radiogenomics: establishing causality between genetics and } \\ \text { radiomics }\end{array}$

Chapter 6 Radiomics approach to evaluate tumor hypoxia on CT

Chapter 7 General discussion and future perspectives

Summary

Valorization addendum

Acknowledgements

Curriculum Vitae

Publications 



\section{Chapter 1}

Introduction and outline 
Cancer is one of the leading causes of mortality worldwide. According to the recent report of GLOBOCAN (International Agency for Research on Cancer) an estimate of 14.1 million new cancer cases and 8.2 million cancer deaths occurred in $2012^{1}$. Most commonly diagnosed cancers were lung, followed by breast and colorectal cancers. Mortality rate was the highest for lung cancer followed by liver cancer and stomach cancer ${ }^{1}$.

Cancer is a complex disease and the evolution of normal cells to a neoplastic state occurs through multiple steps described as the hallmarks of cancer by Hanahan and Weinberg ${ }^{2}$. Cancer cells acquire amongst others the capability to promote growth by overexpressing growth factors and growth factor receptors e.g. overexpression of Epidermal Growth Factor Receptor (EGFR). When the tumor grows, the demand for oxygen and nutrients increase and thus the tumors stimulate signals to develop new blood vessels. When there is insufficient amount of oxygen, the cells become hypoxic. Eventually cancer cells overexpress enzymes (such as MMPs) to degrade the extracellular matrix components and develop a phenotype to facilitate invasion into surrounding tissues and to metastasize to distant organs in the body ${ }^{2}$. A schematic representation of an actively growing and invading tumor is shown in figure 1.

Figure 1: schematic representation of actively growing and invading tumor
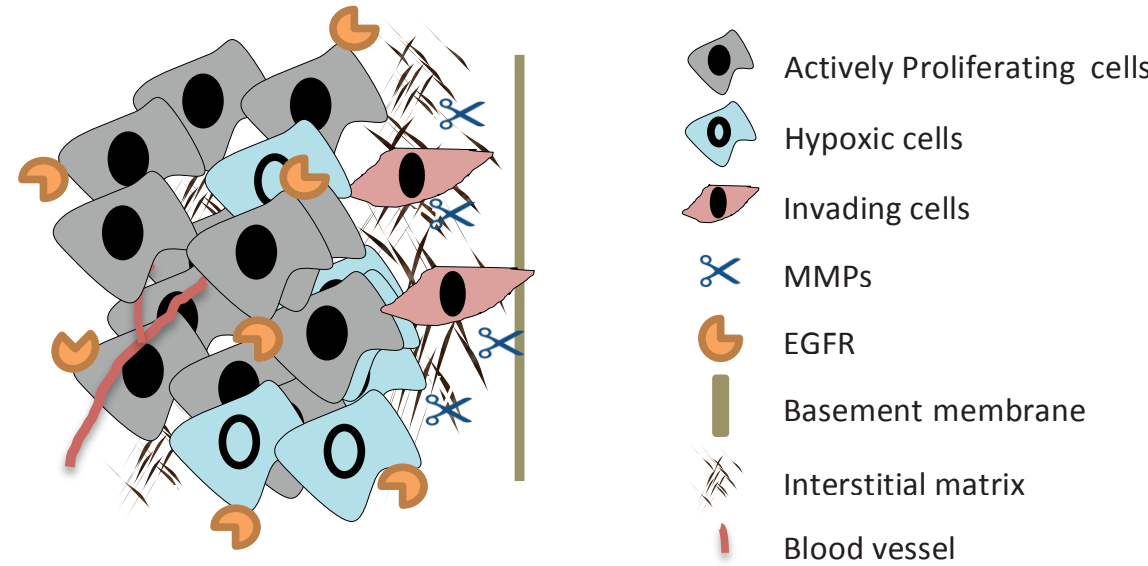

Early diagnosis and treatment of cancer is the major contributor to prevent progression of cancer and to reduce mortality. Treating patients that are not responding to a specific treatment affects the quality of life 
and is also expensive. Therefore, characterization of cancer at a biological level to personalize treatment and thereafter monitoring the patient during the course of treatment is necessary to enhance cancer cure rates. Imaging plays a major role in cancer diagnosis, treatment planning and monitoring treatment responses.

\section{Different imaging modalities}

Unlike invasive biopsies, imaging provides spatial heterogeneity in the tumor noninvasively and longitudinally. There are six broadly used imaging modalities as described below: Magnetic Resonance Imaging (MRI), Computed Tomography (CT), Positron Emission Tomography (PET), Single-Photon Emission Computed Tomography (SPECT), and Ultrasound (US) and Optical Imaging. The later has limited applications in clinic due to its low penetration capability throughout tissues. Table 1 briefly indicates the differences in characteristics of imaging modalities.

Table 1: Comparison of different characteristics of imaging modalities

\begin{tabular}{|l|c|c|c|c|c|c|}
\hline $\begin{array}{l}\text { Molecular } \\
\text { information }\end{array}$ & intermediate & low & $\begin{array}{l}\text { very } \\
\text { high }\end{array}$ & $\begin{array}{c}\text { very } \\
\text { high }\end{array}$ & high & high \\
\hline $\begin{array}{l}\text { Anatomical } \\
\text { information }\end{array}$ & very high & $\begin{array}{l}\text { very } \\
\text { high }\end{array}$ & low & low & high & low \\
\hline Spatial resolution & very high & high & low & low & high & low \\
\hline $\begin{array}{l}\text { Temporal } \\
\text { resolution }\end{array}$ & Low & high & high & high & high & high \\
\hline $\begin{array}{l}\text { Penetration in } \\
\text { Depth }\end{array}$ & High & high & $\begin{array}{c}\text { No } \\
\text { limit }\end{array}$ & $\begin{array}{l}\text { No } \\
\text { limit }\end{array}$ & intermediate & low \\
\hline
\end{tabular}

\section{Magnetic Resonance Imaging}

MRI uses powerful magnets and radiofrequency (RF) waves for visualizing soft tissues and internal structures. When an atom has an equal number of protons and neutrons the atom is neutral and therefore stable. 
However, when there are unequal number of protons and neutrons, the particles are in continuous motion and spin about their own axes, a phenomenon called the angular momentum. Along with this angular momentum particles also possess a magnetic field. Upon exposure to an external magnet, e.g. when a patient is placed inside the MRI scanner, protons behave as magnetic dipoles and align either parallel or antiparallel to the magnetic field. If there are equal numbers of parallel and anti-parallel alignments the resultant magnetic field (net polarization) is zero. Thus, the signal for MRI is generated by differences in parallel and anti-parallel alignments. When a radiofrequency (RF) pulse is applied, perturbation in the alignment of protons occurs which acquire a higher energy state. Subsequently the protons 'relax' back to original state by releasing energy. Two types of relaxation occur: 1 ) longitudinal relaxation (T1 or spin-lattice relaxation) and 2) transverse relaxation (T2 or spin-spin relaxation). The relaxation rates depend on the tissue properties and therefore provide excellent soft tissue contrast compared to $C T$ that measures tissue density. More elaborate description on MR physics can be found elsewhere ${ }^{3-6}$. MRI needs a long time to acquire high quality images as the signal is directly proportional to the net polarization. MRI is mostly used for anatomical information and is based on ${ }^{1} \mathrm{H}$ due to high abundance in the body. In addition, imaging of other nuclei such as ${ }^{13} \mathrm{C}$, ${ }^{31} \mathrm{P},{ }^{19} \mathrm{~F}$ can also be achieved by a technique called magnetic resonance spectroscopy (MRS). With MRS chemical composition of molecules and concentration of the molecules can be determined because of inhomogeneity caused in magnetic field, termed as chemical shift. Imaging $\mathrm{pH}$ of tumors and tumor hypoxia was demonstrated by using ${ }^{31} \mathrm{P}$ and ${ }^{18} F^{7,8}$. MRS can also obtain metabolite information of endogenous molecules such as choline, creatine, lactate etc ${ }^{9-12}$. Other advanced MRI techniques include functional MRI (fMRI), diffusion weighted MRI (DWI) or dynamic contract-enhanced MRI (DCE-MRI) that enables imaging of physiological information such as blood oxygen levels, necrosis and blood vessel information respectively ${ }^{13}$. Recent advancement in MRI is the application of targeted contrast agents (Gadolinium complexes) for molecular imaging ${ }^{13-15}$. The main advantage of MRI compared with other imaging modalities is that it does not require ionizing radiation and has high spatial resolution ${ }^{16}$.

\section{Computed Tomography}

CT imaging relies on the different attenuation properties of the X-rays in different tissues. Tissues absorbing X-rays completely (e.g. bone) appear 
white and tissues poorly attenuating X-rays (e.g. lung) appear black on CT images. Unlike radiographs, CT imaging provides 3D anatomical information by tomographic (different slices through the body) imaging. Attenuation is quantitatively expressed in Hounsfield units (HU), named after Sir Godfrey Hounsfield. HU for air is -1000 , water is 0 and bone is +1000 . Often, contrast agents (e.g. iodine) are used to enhance contrast especially in soft tissues. The attenuation of X-rays in tissues not only depends on the tissue itself but also on the X-ray energy. Therefore, dual energy CT (DECT) uses two different energies, which enables the determination of tissue composition ${ }^{17,18}$. CT imaging is mostly used to obtain anatomical information and has high spatial resolution. CT has poor soft tissue contrast compared to MRI but the acquisition time to obtain CT images is shorter than for MRI. However, due to harmful X-ray radiation, CT scans cannot be performed many times in a patient ${ }^{16}$.

\section{Positron Emission Tomography}

PET use radioactive isotopes and hence is a nuclear imaging method for the detection of different molecular processes ${ }^{19,20}$. Radioactive isotopes such as ${ }^{11} \mathrm{C}$ (half-life 20.3 minutes), ${ }^{18} \mathrm{~F}$ (109.8 minutes), ${ }^{64} \mathrm{Cu}$ (12.7 hours), ${ }^{89} \mathrm{Zr}$ (78.4 hours) etc. are unstable due to extra protons. Due to instability, the excess protons are converted into neutrons, positrons and neutrinos, which are emitted from the nucleus finally resulting in stable atoms. The emitted positron will interact with electrons in the tissue, an event named 'annihilation' occurs, which results in emission of two $511 \mathrm{keV}$ photons in opposite direction. Specially designed ring detectors surrounding the subject can detect these high-energy photons. One of the widely used Food and Drug Administration (FDA) approved PET tracer is ${ }^{18} \mathrm{~F}$-Fluorodeoxyglucose $\left({ }^{18} \mathrm{~F}\right.$-FDG). FDG is a glucose analogue and thus ${ }^{18} \mathrm{~F}$-FDG imaging permits the detection of high metabolism in cancers. ${ }^{18} \mathrm{~F}$ FDG although a widely used tracer, is less specific because most of the cells metabolize glucose. Furthermore, false positive and false negative cases with ${ }^{18}$ F-FDG imaging were reported ${ }^{21-23}$. Therefore, several new imaging biomarkers are being developed for detection of tumors and also to follow-up treatment efficacy for designing improved clinical trials namely window of opportunity trails. For example, EGFR imaging for using ${ }^{89} \mathrm{Zr}$-cetuximab for EGFR targeted treatment or hypoxia evaluation for using ${ }^{18} \mathrm{~F}-\mathrm{HX} 4$ for a hypoxia targeted treatment not only aids in selection of patients for the targeted therapy but also helps in understanding the drug efficacy and distribution in the tumor ${ }^{24}$. 


\section{Single Photon Emission Computed Tomography}

SPECT is similar to PET except that radioisotopes emit single $\curlyvee$-rays. Since the origin of photons is unknown, collimators are used to cut-off diagonally incident photons ${ }^{25}$. Consequently, the sensitivity of SPECT is lower than PET. The main advantage of SPECT over PET is that multiple biological processes can be studied by imaging two radiotracers emitting different energies ${ }^{26}$. SPECT and PET are highly specific and sensitive techniques to detect molecular and physiological processes but have limited spatial resolution compared to $\mathrm{MRI}$ and $\mathrm{CT}^{16}$.

\section{Ultrasound}

US uses properties of sound waves to image tissue and provides anatomical information ${ }^{27}$. A transducer or a probe converts electrical signals to sound waves and emits short pulses of high frequency sound waves that travel through the tissue. Tissue then reflects back the sound waves as echoes, which are dependent on the tissue interface. These echoes are converted back into electric signals to generate an image. Gas filled microbubbles usually coated with lipids can be used as contrast agents. Targeting compounds such as antibodies attached to microbubbles enables molecular imaging and also drug delivery ${ }^{28,29}$. The main advantages are the portability, ease of use in the clinic, employs no harmful radiation source.

\section{Optical imaging}

Optical imaging techniques rely on the use of properties of light to detect molecular processes ${ }^{30}$. When light travels through the medium it undergoes absorption, scattering and emission. Fluorescence and luminescence are two naturally occurring processes which are exploited in optical imaging. Fluorescence is a process when an electron that is laser-excited by absorption of a high-energy photon returns to its ground state by emitting low energy photons. Therefore, a fluorescent probe has a specific excitation wavelength and emission wavelength. Fluorescence imaging in the near infrared range (NIR) has advantages of deeper penetration in tissue due to less absorption ${ }^{31,32}$. On the other hand, bioluminescence is a process where emission of light occurs upon a chemical reaction. Luciferin, a substrate of luciferase is converted to oxy- 
luciferin in the presence of oxygen and adenosine triphosphate (ATP) accompanied by emission of light. For bioluminescence imaging, cells are genetically transformed to produce luciferase. Thus, the emission of light provides the cellular localization and the amount of cells and therefore can monitor tumor growth in preclinical studies. It also provides molecular information when luciferase is expressed along with protein of interest $^{33}$. On the other hand, fluorescence imaging requires cell labeling with fluorescent tags (e.g. GFP tagged cells) to study tumor growth and targeted compounds of interest (antibodies, peptides etc.) coupled to a fluorescent probe to study molecular processes. The emitted light in either fluorescence or bioluminescence is recorded by charge-coupled device (CCD) camera and thus enables to locate the source of light emitted. Optical imaging is highly sensitive technique to study molecular processes in small animals however has limited applications in clinic due to low depth penetration ${ }^{34}$. 


\section{Molecular imaging in cancer theragnostics}

Molecular imaging plays a central role in the context of personalized medicine. Non-invasive imaging of biological processes allows for assessing comprehensive tumor heterogeneity spatially, temporally and longitudinally unlike invasive biopsies ${ }^{35}$. Imaging prior therapy helps to categorize patients for treatment and to monitor treatment efficacy. Imaging of therapeutic antibodies/drugs gives further understanding on the drug stability, kinetics and drug uptake in the tumors and normal tissues, which might aid in drug development. The term theragnostics is coined to define the combination of both therapy and diagnosis ${ }^{36}$. Theragnostic approach aids in implementation of improved clinical trials such as window of opportunity trials for more patient specific treatment and to reduce the delays in treatment. In a window of opportunity trial, patients who can potentially respond to a new drug agree to receive the new treatment before undergoing the conventional treatment.

For individualized patient treatment, selection of the patient should be done based on the tumor molecular characterization. ${ }^{18}$ F-FDG PET imaging is widely used and helps in tumor staging. However, false positive or false negative findings were also reported especially post treatment due to inflammation ${ }^{37-40}$. When inhibiting proliferation using drugs such as cisplatin or oxaliplatin it would be ideal to use a proliferation biomarker for imaging such as ${ }^{18} \mathrm{~F}_{-} \mathrm{FLT}^{41,42}$. Similarly, when evaluating hypoxia-targeted therapy, it would be ideal to employ hypoxia imaging such as ${ }^{18} \mathrm{~F}$-HX4 PET or ${ }^{18} \mathrm{~F}$-FMISO PET that can ideally provide the hypoxic regions within the tumor before and after therapy ${ }^{43,44}$.

\section{Epidermal growth factor receptor (EGFR)}

EGFR is a membrane tyrosine kinase receptor belonging to the ErbB family and has an extracellular domain for ligand binding, a transmembrane domain for regulatory mechanisms such as ligand internalization and a cytoplasmic domain for signal transduction (tyrosine kinase activity) ${ }^{45}$. EGFR (also called ErbB-1/ HER1) has a number of activating ligands including epidermal growth factor (EGF), transforming growth factor alpha (TGF- $\alpha$ ) and neuregulins ${ }^{46}$. EGFR expression levels are elevated in many cancers including non-small cell lung cancer (NSCLC), head and neck, colorectal, breast, cervical and ovarian cancers ${ }^{47}$. The level of EGFR expression has been directly linked to tumor aggressiveness, poor treatment outcome and worse prognosis ${ }^{48,49}$. EGFR 
overexpression and signaling has been linked to the invasiveness of cancers $^{50,51}$ and it was reported that cytoplasmic EGFR overexpression was found in high grade and invasive pancreatic ducal adenocarcinoma ${ }^{51}$. EGFR overexpression can occur due to different mechanisms including mutations causing constitutive activation of the receptor signaling, increased expression of ligands or heterodimerization with other receptors of the ErBb family (EGFR-HER2 heterodimerization) ${ }^{45}$. Due to high expression of EGFR in many cancers, a number of therapeutic molecules have been developed including small molecule kinase inhibitors (e.g. gefitinib, erlotinib) and monoclonal antibodies (e.g. cetuximab). For treatment purposes, patients are usually categorized as EGFR positive or EGFR negative. EGFR expression is usually evaluated by immunohistochemistry $(\mathrm{IHC})$ providing not only information on total EGFR levels but also on its distribution. Other measures such as determining RNA levels and protein expression by western blotting are also commonly used ${ }^{52}$, however information on heterogeneity of expression is lost. Moreover, reports on EGFR as a prognostic marker varied largely because of inconsistency in methods to evaluate EGFR expression $^{52}$. Furthermore, studies have reported a disparity between EGFR expression and outcome after EGFR-targeted therapy ${ }^{53-55}$. Imaging of EGFR expression with EGFR targeting therapeutic molecules such as cetuximab could be an alternative theragnostic approach enabling determination of the amount of antibody reaching the tumor before starting therapy. Previously, our group has demonstrated that a disparity exists between EGFR expression and cetuximab uptake in tumors indicating that other parameters might influence cetuximab uptake and hence treatment response ${ }^{56}$. Since antibody uptake in tumors is generally dependent on tumor microenvironmental parameters ${ }^{57}$, it is essential to determine if these parameters influence the uptake of cetuximab. Imaging cetuximab antibody in different tumors with varying EGFR expression and determining the factors influencing the uptake of antibody might provide an answer for the discrepancies between cetuximab treatment efficacy and EGFR expression.

\section{Matrix metallo proteinase 2}

Matrix metalloproteinases also called Matrixins are a family of $\mathrm{Zn}^{2+}$ containing endopeptidases that are vital for the tissue remodeling and breakdown of extracellular matrix components (ECM) such as gelatin, collagen, elastin, glycoproteins and proteoglycans ${ }^{58,59}$. MMPs, which are present in low levels in normal conditions, are highly expressed during 
pathological conditions including cancers ${ }^{60,61}$. Tumor microenvironmental parameters like hypoxia regulate MMPs and their localization. MMP2 (gelatinase $A$ ) is one of the most extensive studied MMPs and its role in various processes of cancer progression such as tumorigenesis, angiogenesis and tumor invasion was reported. Its increased expression was often associated with aggressive cancer phenotypes like glioblastoma $^{62}$, melanomas ${ }^{63}$, breast cancer $^{64}$ and lung cancers ${ }^{65}$. Circulating levels of MMP2 were detected in patients with late stages of cancer $^{66-68}$. MMP2 was not only associated with tumor invasion but also involved in angiogenesis ${ }^{69,70}$. Inhibition of MMP2 led to a reduced vessel density, reduced tumor growth and decrease in invasiveness ${ }^{71-73}$. Therefore, MMP2 might be a potential therapeutic target as well as imaging biomarker for detecting invasive phenotypes.

Optical imaging techniques have been widely used for the measurement of MMP2 activity in vivo. Optically silent probes emit fluorescence when cleaved by MMP2 and thus represent localized MMP2 activity. Optical silence is achieved either by fluorophore-fluorophore pairing or fluorophore-quencher pairing resulting in Forster Resonance Energy Transfer (FRET). The first study that could measure MMP2 activity in vivo was done by Bremer et al using activatable probes ${ }^{74}$. However, the clinical translation of these molecules is difficult due to bulky nature of the molecules attached to the cleavable peptide and limited application of optical imaging in clinic. Furthermore, cleavage of the probe can also occur by other proteases decreasing the specificity towards MMP2. On the other hand, imaging with antibodies or inhibitors against MMP2 allows for theragnostic applications. High specificity of antibody towards the target aids in accurate detection of the target. Therefore, it might be possible to specifically determine MMP2 in tumors using antibody targeting active MMP2.

\section{Imaging features: More than just images}

$\mathrm{CT}$ and MRI are mainly used to obtain anatomical information. However, recent studies have shown that these medical images contain more information than visible and detectable by the human eye. This information can be quantified by advanced image analysis methodology. Radiomics, an example of such an advanced image analysis method, extracts large number of features from medical images based on pixel intensities, shape and size and texture (distribution of gray level intensities). Imaging features were shown to provide prognostic 
information in human cancers ${ }^{75-77}$. It was reported that radiomics signatures built from pixel intensity (statistics energy), shape (compactness) and texture (gray level non-uniformity) were prognostic in lung and head and neck cancer patients and were associated with gene expression profiles ${ }^{78}$. Furthermore, radiomics features derived from texture (defining tumor heterogeneity) and pixel intensity (defining asymmetry of mean pixel intensity) were also found to be predictive for metastatic phenotype in lung cancer patients ${ }^{79}$. Besides, quantitative PET imaging features were shown to provide prognostic information in lung cancer patients, i.e. radiomics feature determined as relative volume above $80 \%$ of SUV was shown to be an independent prognostic factor and not the commonly used SUV values (SUVpeak, SUVmax and SUVmean) ${ }^{80}$. In addition, studies have shown that imaging features were able to detect drug responsive patterns in hepatocellular carcinoma patients indicating that evaluating treatment responses is potentially possible by quantifying heterogeneities by using image derived features ${ }^{81}$. Radiomics is currently an active field and widely reported in clinical studies. Studies have shown clear associations between imaging features and gene expression profiles ${ }^{82}$. However, the causal relation of features with the underlying biology and tumor heterogeneity is not fully established. Therefore there is a need to categorize features that are ideally responsible for a characteristic phenotype in tumors. Since current imaging modalities only provide anatomical and/or molecular information, imaging features derived from anatomical images (CT/MRI) might act as a bridge and provide information beyond anatomy and molecular details (Figure 2). If the underlying biological relationship of the imaging features is established, radiomics might ideally be used for quantifying both anatomical and molecular information that can predict treatment responses.

Figure 2

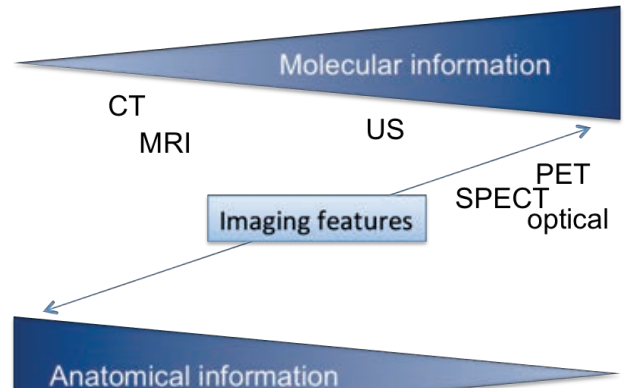




\section{Outline of the thesis}

In this thesis, we aimed to develop novel molecular imaging methods that can eventually provide an opportunity for improved clinical designs such as 'window-of-opportunity' trials for individualized treatment. In the first part of the thesis, we evaluated novel molecular imaging tracers that can potentially aid in drug development and also theragnosis. In the second part of the thesis, we determined the association of CT-derived radiomics features with tumor heterogeneities that would eventually aid in providing molecular information without the need for radioactive tracers (Figure 3).

\section{Figure 3}

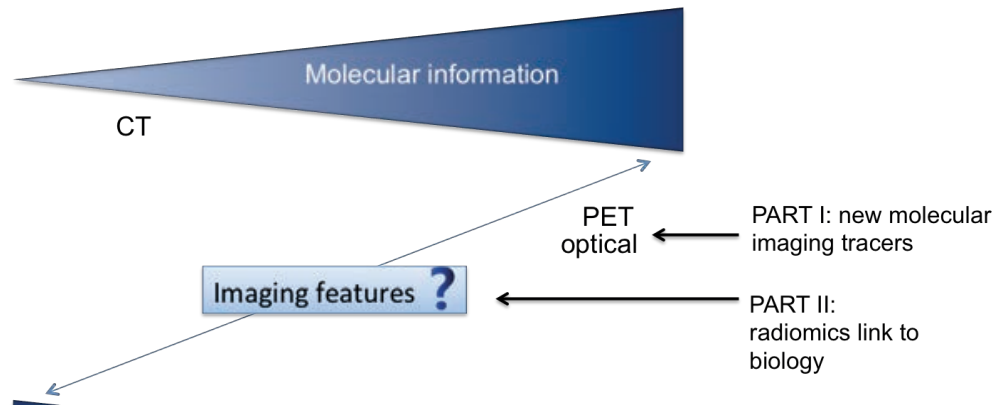

In chapter 2, we discussed about the new possibilities for imaging and targeting hypoxia. Moreover, the potential of non-invasive imaging in exploring window-of-opportunity trials for personalized treatment and drug development is discussed. Uptake of ${ }^{89} \mathrm{Zr}$ labeled Cetuximab in patient derived xenograft tumor models with varying EGFR expression levels was evaluated by PET imaging. Previously it was shown by our group that the uptake of ${ }^{89} \mathrm{Zr}$ labeled cetuximab was not solely dependent on EGFR expression. Hence, in chapter 3, we evaluated if tumor microenvironmental parameters such as vessel density and hypoxia influenced the uptake of the antibody. Next, we evaluated a novel MMP2 antibody as an imaging biomarker since MMP2 is highly expressed in invasive cancers. Therefore, in chapter 4 we evaluated the potential use of MMP2 small immuno protein (aMMP2-SIP) antibody as an imaging 
biomarker using near infrared fluorescence imaging after tagging the SIP with Cy5. To evaluate the antibody uptake genetic MMP2 knockdown models were designed and validated.

In second part (chapter 5 and 6), we determined if a causal link exists between imaging features and tumor heterogeneities using preclinical datasets. In chapter 5, we evaluated if a genetic change would causally affect radiomics features. For this, tumors with known genetic change (upregulation of GADD34c) were grown in mice and CT imaging was performed at several time points. CT image features were extracted and compared before and post gene induction. Additionally, effect of radiation treatment in both gene-induced and control tumors was determined. In chapter $\mathbf{6}$, the capability of radiomics features in determining the oxygenation status of tumors was evaluated by comparing radiomics features to the hypoxia PET tracer ${ }^{18} \mathrm{~F}-\mathrm{HX} 4$ uptake in tumors.

Finally in chapter $\mathbf{7}$, the results of previously described chapters and future perspectives are discussed. 


\section{References}

Ferlay, J. et al. Cancer incidence and mortality worldwide: Sources, methods and major patterns in GLOBOCAN 2012. International Journal of Cancer 136, E359E386, doi:10.1002/ijc.29210 (2015).

Hanahan, D. \& Weinberg, R. A. The Hallmarks of Cancer. Cell 100, 57-70, doi:http://dx.doi.org/10.1016/S0092-8674(00)81683-9 (2000).

Edelman, R. R. \& Warach, S. Magnetic resonance imaging. New England Journal of Medicine 328, 708-716 (1993).

Levitt, M. H. Spin dynamics: basics of nuclear magnetic resonance. (John Wiley \& Sons, 2001).

Joy, M., Scott, G. \& Henkelman, M. In vivo detection of applied electric currents by magnetic resonance imaging. Magnetic Resonance Imaging 7, 89-94 (1989).

Bloch, F. Nuclear induction. Physical review 70, 460 (1946).

Salmon, H. W. \& Siemann, D. W. Utility of 19F MRS detection of the hypoxic cell marker EF5 to assess cellular hypoxia in solid tumors. Radiotherapy and Oncology 73, 359-366 (2004).

Gillies, R., Liu, Z. \& Bhujwalla, Z. 31P-MRS measurements of extracellular pH of tumors using 3-aminopropylphosphonate. American Journal of Physiology-Cell Physiology 267, C195-C203 (1994).

Miller, B. L. et al. In vivo $1 \mathrm{H}$ MRS choline: Correlation with in vitro chemistry/histology. Life Sciences 58, 1929-1935 (1996).

Utriainen, M. et al. Evaluation of brain tumor metabolism with [11C]choline PET and 1H-MRS. Journal of Neuro-Oncology 62, 329-338, doi:10.1023/a:1023342516925 (2003).

Gujar, S. K., Maheshwari, S., Björkman-Burtscher, I. \& Sundgren, P. C. Magnetic resonance spectroscopy. Journal of neuro-ophthalmology 25, 217-226 (2005).

Duncan, J. S. Magnetic resonance spectroscopy. Epilepsia 37, 598-605 (1996).

Gallagher, F. A. An introduction to functional and molecular imaging with MRI. Clinical Radiology 65, 557-566 (2010).

Shuhendler, A. J. et al. Molecular Magnetic Resonance Imaging of Tumor Response to Therapy. Scientific Reports 5, 14759, doi:10.1038/srep14759 (2015). Hengerer, A. \& Grimm, J. Molecular magnetic resonance imaging. Biomedical Imaging and Intervention Journa/ 2, e8, doi:10.2349/biij.2.2.e8 (2006).

James, M. L. \& Gambhir, S. S. A Molecular Imaging Primer: Modalities, Imaging Agents, and Applications. Physiological Reviews 92, 897-965, doi:10.1152/physrev.00049.2010 (2012).

Schmid-Bindert, G. et al. Functional imaging of lung cancer using dual energy CT: how does iodine related attenuation correlate with standardized uptake value of 18FDG-PET-CT? European Radiology 22, 93-103, doi:10.1007/s00330-011-2230-3 (2012).

van Elmpt, W., Zegers, C. M. L., Das, M. \& De Ruysscher, D. Imaging techniques for tumour delineation and heterogeneity quantification of lung cancer: overview of current possibilities. Journal of Thoracic Disease 6, 319-327, doi:10.3978/j.issn.2072-1439.2013.08.62 (2014).

Phelps, M. E. PET: The Merging of Biology and Imaging into Molecular Imaging. Journal of Nuclear Medicine 41, 661-681 (2000).

Wernick, M. N. \& Aarsvold, J. N. Emission tomography: the fundamentals of PET and SPECT. (Academic Press, 2004).

Rosenbaum, S., Lind, T., Antoch, G. \& Bockisch, A. False-Positive FDG PET Uptake-the Role of PET/CT. European Radiology 16, 1054-1065, doi:10.1007/s00330-005-0088-y (2006). 
Inoue, T. et al. Detecting Recurrent or Residual Lung Cancer with FDG-PET. Journal of Nuclear Medicine 36, 788-793 (1995). Lung Carcinoma. Journal of Nuclear Medicine 39, 1016-1020 (1998). Orloff, J. et al. The future of drug development: advancing clinical trial design. Nat Rev Drug Discov 8, 949-957 (2009). Madsen, M. T. Recent Advances in SPECT Imaging. Journal of Nuclear Medicine 48, 661-673, doi:10.2967/jnumed.106.032680 (2007). Imaging: An Overview. International Journal of Molecular Imaging 2011, doi:10.1155/2011/796025 (2011).

Chan, V. \& Perlas, A. in Atlas of Ultrasound-Guided Procedures in Interventional Pain Management (ed Samer N. Narouze) Ch. 2, 13-19 (Springer New York, 2011).

Gessner, R. \& Dayton, P. A. Advances in molecular imaging with ultrasound. Molecular imaging 9, 117-127 (2010).

9 Unnikrishnan, S. \& Klibanov, A. L. Microbubbles as Ultrasound Contrast Agents for Molecular Imaging: Preparation and Application. American Journal of Roentgenology 199, 292-299, doi:10.2214/ajr.12.8826 (2012).

Leff, D. et al. Diffuse optical imaging of the healthy and diseased breast: A systematic review. Breast Cancer Research and Treatment 108, 9-22, doi:10.1007/s10549-007-9582-z (2008).

Frangioni, J. V. In vivo near-infrared fluorescence imaging. Current Opinion in Chemical Biology 7, 626-634 (2003).

Chen, X., Conti, P. S. \& Moats, R. A. In vivo Near-Infrared Fluorescence Imaging of Integrin $\alpha v \beta 3$ in Brain Tumor Xenografts. Cancer Research 64, 8009-8014, doi:10.1158/0008-5472.can-04-1956 (2004). Rehemtulla, A. et al. Rapid and Quantitative Assessment of Cancer Treatment Response Using In Vivo Bioluminescence Imaging. Neoplasia 2, 491-495 (2000). Solomon, M., Liu, Y., Berezin, M. Y. \& Achilefu, S. Optical Imaging in Cancer Research: Basic Principles, Tumor Detection, and Therapeutic Monitoring. Medical Principles and Practice 20, 397-415 (2011). Massoud, T. F. \& Gambhir, S. S. Molecular imaging in living subjects: seeing fundamental biological processes in a new light. Genes \& development 17, 545580 (2003).

Bioconjugate Chemistry 22, 1879-1903, doi:10.1021/bc200151q (2011). Purandare, N. C., Puranik, A. D., Shah, S., Agrawal, A. \& Rangarajan, V. Posttreatment appearances, pitfalls, and patterns of failure in head and neck cancer on FDG PET/CT imaging. Indian Journal of Nuclear Medicine : IJNM : The Official Journal of the Society of Nuclear Medicine, India 29, 151-157, doi:10.4103/09723919.136564 (2014).

8 Zhu, A., Lee, D. \& Shim, H. Metabolic PET Imaging in Cancer Detection and Therapy Response. Seminars in oncology 38, 55-69, doi:10.1053/j.seminoncol.2010.11.012 (2011).

Purohit, B. et al. FDG-PET/CT pitfalls in oncological head and neck imaging. Insights into Imaging 5, 585-602, doi:10.1007/s13244-014-0349-x (2014).

van Waarde, A. et al. Selectivity of 18F-FLT and 18F-FDG for Differentiating Tumor from Inflammation in a Rodent Model. Journal of Nuclear Medicine 45, 695-700 (2004).

Buck, A. K. et al. Imaging Proliferation in Lung Tumors with PET: 18F-FLT Versus 18F-FDG. Journal of Nuclear Medicine 44, 1426-1431 (2003). 

Deoxy-3'-[18F]Fluorothymidine Positron Emission Tomography: The Effect of Cisplatin on a Fibrosarcoma Tumor Model In vivo. Cancer Research 65, 42024210, doi:10.1158/0008-5472.can-04-4008 (2005). Peeters, S. G. J. A. et al. TH-302 in Combination with Radiotherapy Enhances the Therapeutic Outcome and Is Associated with Pretreatment [18F]HX4 Hypoxia PET Imaging. Clinical Cancer Research 21, 2984-2992, doi:10.1158/1078-0432.ccr-150018 (2015). Positron Emission Tomography in Head and Neck Cancer. Clinical Cancer Research 12, 5435-5441, doi:10.1158/1078-0432.ccr-05-1773 (2006). Jorissen, R. N. et al. Epidermal growth factor receptor: mechanisms of activation and signalling. Experimental Cell Research 284, 31-53 (2003).

46 Normanno, N. et al. Epidermal growth factor receptor (EGFR) signaling in cancer. Gene 366, 2-16 (2006).

47 Salomon, D. S., Brandt, R., Ciardiello, F. \& Normanno, N. Epidermal growth factor-related peptides and their receptors in human malignancies. Critical Reviews in Oncology/Hematology 19, 183-232 (1995). Selvaggi, G. et al. Epidermal growth factor receptor overexpression correlates with a poor prognosis in completely resected non-small-cell lung cancer. Annals of Oncology 15, 28-32, doi:10.1093/annonc/mdh011 (2004). Nicholson, R. I., Gee, J. M. W. \& Harper, M. E. EGFR and cancer prognosis. European Journal of Cancer 37, Supplement 4, 9-15 (2001).

50 Mader, C. C. et al. An EGFR-Src-Arg-Cortactin Pathway Mediates Functional Maturation of Invadopodia and Breast Cancer Cell Invasion. Cancer Research 71, 1730-1741, doi:10.1158/0008-5472.can-10-1432 (2011).

51 Ueda, S. et al. The correlation between cytoplasmic overexpression of epidermal growth factor receptor and tumor aggressiveness: poor prognosis in patients with pancreatic ductal adenocarcinoma. Pancreas 29, e1-e8 (2004). Arteaga, C. L. Epidermal Growth Factor Receptor Dependence in Human Tumors: More Than Just Expression? The Oncologist 7, 31-39, doi:10.1634/theoncologist.7-suppl_4-31 (2002).

Scaltriti, M. \& Baselga, J. The Epidermal Growth Factor Receptor Pathway: A Model for Targeted Therapy. Clinical Cancer Research 12, 5268-5272, doi:10.1158/1078-0432.ccr-05-1554 (2006).

54 Scartozzi, M. et al. Epidermal Growth Factor Receptor (EGFR) Status in Primary Colorectal Tumors Does Not Correlate With EGFR Expression in Related Metastatic Sites: Implications for Treatment With EGFR-Targeted Monoclonal Antibodies. Journal of Clinical Oncology 22, 4772-4778, doi:10.1200/jco.2004.00.117 (2004).

55 Parra, H. S. et al. Analysis of epidermal growth factor receptor expression as a predictive factor for response to gefitinib (/'Iressa/', ZD1839) in non-small-cell lung cancer. Br J Cancer 91, 208-212 (2004).

56 Aerts, H. J. W. L. et al. Disparity Between In Vivo EGFR Expression and 89ZrLabeled Cetuximab Uptake Assessed with PET. Journal of Nuclear Medicine 50, 123-131, doi:10.2967/jnumed.108.054312 (2009).

57 Jain, R. K. Physiological Barriers to Delivery of Monoclonal Antibodies and Other Macromolecules in Tumors. Cancer Research 50, 814s-819s (1990).

58 Nagase, H. \& Woessner, J. F. Matrix Metalloproteinases. Journal of Biological Chemistry 274, 21491-21494, doi:10.1074/jbc.274.31.21491 (1999).

59 Birkedal-Hansen, H. et al. Matrix Metalloproteinases: A Review. Critical Reviews in Oral Biology \& Medicine 4, 197-250, doi:10.1177/10454411930040020401 (1993). 
Rundhaug, J. E. Matrix Metalloproteinases, Angiogenesis, and Cancer: Commentary re: A. C. Lockhart et al., Reduction of Wound Angiogenesis in Patients Treated with BMS-275291, a Broad Spectrum Matrix Metalloproteinase Inhibitor. Clin. Cancer Res., 9: 00-00, 2003. Clinical Cancer Research 9, 551-554 (2003).

61 Kessenbrock, K., Plaks, V. \& Werb, Z. Matrix Metalloproteinases: Regulators of the Tumor Microenvironment. Cell 141, 52-67 (2010).

Sawaya, R. et al. Expression and localization of $72 \mathrm{kDa}$ type IV collagenase (MMP2) in human malignant gliomas in vivo. Clinical \& Experimental Metastasis 14, 3542, doi:10.1007/bf00157684 (1996). Prognostic value of MMP-2 immunoreactive protein ( $72 \mathrm{kD}$ type IV collagenase) in primary skin melanoma. The Journal of Pathology 186, 51-58, doi:10.1002/(sici)1096-9896(199809)186:1<51::aid-path131>3.0.co;2-p (1998).

Azzam, H. S., Arand, G., Lippman, M. E. \& Thompson, E. W. Association of MMP-2 Activation Potential With Metastatic Progression in Human Breast Cancer Cell Lines Independent of MMP-2 Production. Journal of the National Cancer Institute 85, 1758-1764, doi:10.1093/jnci/85.21.1758 (1993).

Passlick, B. et al. Overexpression of Matrix Metalloproteinase 2 Predicts
Unfavorable Outcome in Early-Stage Non-Small Cell Lung Cancer. Clinical Cancer
Research 6, 3944-3948 (2000).

Rocca, G. L., Pucci-Minafra, I., Marrazzo, A., Taormina, P. \& Minafra, S. Zymographic detection and clinical correlations of MMP-2 and MMP-9 in breast cancer sera. Br J Cancer 90, 1414-1421 (2004).

7 Tutton, M. G. et al. Use of plasma MMP-2 and MMP-9 levels as a surrogate for tumour expression in colorectal cancer patients. International Journal of Cancer 107, 541-550, doi:10.1002/ijc.11436 (2003).

8 Patel, B. P., Shah, S. V., Shukla, S. N., Shah, P. M. \& Patel, P. S. Clinical significance of MMP-2 and MMP-9 in patients with oral cancer. Head \& Neck 29, 564-572, doi:10.1002/hed.20561 (2007).

9 Stetler-Stevenson, W. G. Matrix metalloproteinases in angiogenesis: a moving target for therapeutic intervention. Journal of Clinical Investigation 103, 12371241 (1999).

0 Rojiani, M. V., Alidina, J., Esposito, N. \& Rojiani, A. M. Expression of MMP-2 correlates with increased angiogenesis in CNS metastasis of lung carcinoma. International Journal of Clinical and Experimental Pathology 3, 775-781 (2010).

1 Prontera, C., Mariani, B., Rossi, C., Poggi, A. \& Rotilio, D. Inhibition of gelatinase A (MMP-2) by batimastat and captopril reduces tumor growth and lung metastases in mice bearing Lewis lung carcinoma. International Journal of Cancer 81, 761766, doi:10.1002/(sici)1097-0215(19990531)81:5<761::aid-ijc16>3.0.co;2-1 (1999).

72 Kaliski, A. et al. Angiogenesis and tumor growth inhibition by a matrix metalloproteinase inhibitor targeting radiation-induced invasion. Molecular Cancer Therapeutics 4, 1717-1728, doi:10.1158/1535-7163.mct-05-0179 (2005).

73 Kargiotis, O. et al. Adenovirus-mediated transfer of siRNA against MMP-2 mRNA results in impaired invasion and tumor-induced angiogenesis, induces apoptosis in vitro and inhibits tumor growth in vivo in glioblastoma. Oncogene 27, 48304840, (2008).

74 Bremer, C., Bredow, S., Mahmood, U., Weissleder, R. \& Tung, C.-H. Optical Imaging of Matrix Metalloproteinase-2 Activity in Tumors: Feasibility Study in a Mouse Model. Radiology 221, 523-529, doi:10.1148/radiol.2212010368 (2001). 
Lung and Head \&amp; Neck cancer. Scientific Reports 5, 11044, doi:10.1038/srep11044 (2015).

76 Pope, W. B. et al. MR Imaging Correlates of Survival in Patients with High-Grade Gliomas. American Journal of Neuroradiology 26, 2466-2474 (2005).

77 Gutman, D. A. et al. MR Imaging Predictors of Molecular Profile and Survival: Multi-institutional Study of the TCGA Glioblastoma Data Set. Radiology 267, 560569, doi:10.1148/radiol.13120118 (2013).

78 Aerts, H. J. W. L. et al. Decoding tumour phenotype by noninvasive imaging using a quantitative radiomics approach. Nat Commun 5, doi:10.1038/ncomms5006 (2014).

79 Coroller, T. P. et al. CT-based radiomic signature predicts distant metastasis in lung adenocarcinoma. Radiotherapy and Oncology 114, 345-350, doi:http://dx.doi.org/10.1016/j.radonc.2015.02.015 (2015).

80 Carvalho, S. et al. Prognostic value of metabolic metrics extracted from baseline positron emission tomography images in non-small cell lung cancer. Acta Oncologica 52, 1398-1404, doi:10.3109/0284186x.2013.812795 (2013).

81 Kuo, M. D., Gollub, J., Sirlin, C. B., Ooi, C. \& Chen, X. Radiogenomic analysis to identify imaging phenotypes associated with drug response gene expression programs in hepatocellular carcinoma. Journal of Vascular and Interventional Radiology 18, 821-830 (2007).

82 Karlo, C. A. et al. Radiogenomics of Clear Cell Renal Cell Carcinoma: Associations between CT Imaging Features and Mutations. Radiology 270, 464-471, doi:10.1148/radiol.13130663 (2013). 


\section{Chapter 2}

New ways to image and target tumor hypoxia and its molecular responses

Published in: Radiother Oncol. 2015 Sep; 116(3):352-7

Dubois LJ, Niemans R, van Kuijk SJ, Panth KM, Parvathaneni NK, Peeters SG, Zegers CM, Rekers NH, van Gisbergen MW, Biemans R, Lieuwes NG, Spiegelberg L, Yaromina A, Winum JY, Vooijs M, Lambin P 


\section{Abstract}

Tumor hypoxia and its molecular responses have been shown to be associated with poor prognosis. Detection of hypoxia, preferably in a noninvasive manner, could therefore predict treatment outcome and serve as a tool to individualize treatment. This review gives an overview of recent literature on hypoxia imaging markers currently used in clinical trials. Furthermore, recent progress made in targeting hypoxia (hypoxiaactivated prodrugs) or hypoxia response (carbonic anhydrase IX inhibitors) is summarized. Last, window-of-opportunity trials implementing non-invasive imaging are proposed as an important tool to prove anti-tumor efficacy of experimental drugs early during drug development. 


\section{Introduction}

It is well established that tumors are not a collection of relatively homogeneous cancer cells, but act as organs with a complexity that might even exceed that of healthy tissues. Therefore to understand the biology of a tumor both the different individual cell types within a tumor as well as its microenvironment need to be studied [1]. Within this review, we will focus on hypoxia, a common characteristic of solid tumors, which has been associated with poor prognosis [2]. Detection of hypoxia, preferably in a non-invasive manner, could predict treatment outcome and serve as a tool to support treatment decisions. Such non-invasive imaging approaches that are routinely available in clinical practice including positron emission tomography (PET), magnetic resonance imaging (MRI) and perfusion computed tomography (CT) are able to accurately and reliably image hypoxia in tumors. Over the last decade, these diagnostic techniques are developing into versatile tools integrated in treatment monitoring, outcome prediction and treatment targeting. A meta-analysis evaluating the relationship between hypoxia imaging and outcome after radiation treatment demonstrated a uniform tendency for poor response when tumors were hypoxic. This was not only observed for widely used hypoxic PET tracers, but also when hypoxia was indirectly evaluated using perfusion-CT or DCE-MRI [3].

While the prognostic significance of tumoral hypoxia on outcome has been established more than two decades ago only recently compounds are being tested in clinical trials that enabling monitoring and selective elimination of hypoxic tumors cells. Here will provide an update on the current status of hypoxia imaging agents and strategies to combat tumor hypoxia.

\section{Hypoxia PET imaging tracers}

Multiple PET tracers suitable for the detection of hypoxia have been developed, validated and shown to exhibit different characteristics. The ideal hypoxia tracer has complete clearance of unbound tracer at time of imaging, thus only bound in oxygen deprived tissues resulting in high signal to noise ratios [3]. We recently reviewed the PET hypoxia tracers that were validated in preclinical and clinical studies and reported accurate quantification methods and clinical applications [4]. The most investigated PET hypoxia tracer is fluoromisonidazole (FMISO). However, due to concerns regarding FMISO stability, metabolite formation and slow clearance properties $[5,6]$, alternative hypoxia PET tracers with different clearance and hydrophilicity characteristics have been developed trying 
to overcome these limitations: fluoroazomycin arabinoside (FAZA), fluoroerythronitroimidazole (FETNIM) and fluorinated etanidazole derivatives (EF1, EF3, EF5), which all have been extensively reviewed previously $[3,4,7]$.

More recently, the hydrophilic flortanidazole (HX4), with preferred pharmacokinetics and clearance properties, has been synthesized through click-chemistry [8] showing $82 \%$ intact and $84 \%$ unmetabolized tracer at 2 hours post injection (h p.i.) in plasma and urine respectively [9]. HX4 has been evaluated in a preclinical rhabdomyosarcoma rat tumor model, where binding was causally dependent on tumoral oxygenation status. Furthermore, a significant spatial relationship at tumormicroregional level between HX4 distribution and the exogenous hypoxia marker pimonidazole staining was observed $[10,11]$. Studies in primates and healthy volunteers [9] and in patients with histologically proven solid cancer [12] provided evidence for a good safety profile. Recently, in nonsmall cell lung cancer patients, image contrast was found to be superior $4 \mathrm{~h}$ p.i. compared with earlier time points and uptake patterns were strongly correlated between two scans [13]. Overlap studies between HX4 and the metabolism tracer FDG indicated that on average $24 \%$ of the hypoxic volume is outside the FDG volume [14]. Similar results have been obtained for head and neck cancer patients [15], suggesting that hypoxia PET imaging provides complementary information to FDG imaging.

Due to the large heterogeneity in uptake, differences in tumor and animal models, different time point of imaging and anaesthesia observed in the literature, it is difficult to compare different hypoxia markers. Although characterization of new hypoxia markers should be preferably performed in multiple cancer models, highly additive data can be expected from comparisons of different tracers within the same tumor models $[3,11]$. Recently, we performed a comparative study characterizing the clinically approved hypoxia markers FMISO, FAZA and HX4 on tumor to blood ratio (TBR), reproducibility and reversibility within a rat rhabdomyosarcoma model [16]. Blood clearance for FAZA and HX4 became similar $3 \mathrm{~h}$ p.i., while for FMISO as expected clearance from normal tissues was significantly lower. Differences in tumor uptake resulted in significantly higher TBR for HX4 compared to the other tracers. Reproducibility and spatial overlap between two PET acquisitions over a 48 hour time period was high for both FMISO and HX4. Furthermore, decreasing the hypoxic fraction using carbogen resulted in loss of FMISO uptake, while increased hypoxia achieved by breathing 7\% oxygen, further enhanced FAZA and HX4 uptake. Another study performed a similar comparison in a SQ20b head and neck xenograft mouse model and found similar tumor to muscle 
ratios for FMISO, FAZA and HX4 [10]. However, these results were obtained at $80-90$ minutes p.i., a time point which is probably too early for evaluation since normal tissue clearance is still ongoing. A comparative study in head and neck cancer patients found similar tumor to muscle ratios for $\mathrm{HX} 4$ imaging at $1.5 \mathrm{~h} \mathrm{p.i.} \mathrm{and} \mathrm{FMISO} \mathrm{imaging} \mathrm{at} 2 \mathrm{~h} \mathrm{p.i.}$ [17]. For HX4 higher uptake and increasing ratios would be expected at later time points based on our clinical results [13]. Recently a simulation study, comparing FMISO, FAZA and HX4 based on their respective physical and chemical properties, revealed that tracer clearance and diffusion are the major parameters influencing image contrast. Highest clearance and image contrast was observed for HX4, but also the largest patient-to-patient variation, which might be a concern for clinical imaging to define tumor hypoxia based on a reliable threshold value [18].

Current available tracers have proven to be reliable for evaluation of tumor hypoxia, although with inherent problems resulting in clinical limitations. Alternative tracers, such as $\mathrm{HX} 4$, are promising with respect to deliver higher contrast images, whereas FMISO remains a robust reproducible hypoxia marker. It is not inconceivable that more tracers will be developed; but currently existing PET tracers should rather be used in clinic with standardized protocols enabling comparisons between different institutes. Furthermore, applicability and clinical validation should be proven in multiple cancer types and tracers need be tested with respect to their prognostic and predictive value.

\section{Hypoxia targeting}

The compelling evidence for hypoxia in tumor tissue and its therapeutic importance makes hypoxia a high priority target for cancer therapy. Bioreductive prodrugs selectively activated under hypoxia and drugs that inhibit molecular targets in hypoxic cells (vide infra) are currently extensively investigated. A recent overview described the challenged and opportunities of these strategies [19]. The clinically most advanced hypoxia-activated prodrug is tirapazamine (TPZ). Although promising results have been reported in a number of Phase 2 trials, TPZ failed in several Phase 3 clinical trials since no survival benefit was observed when incorporated into standard therapy regimens. Possible explanations are its poor tumor penetration, low in vivo potency at tolerable doses and unacceptable toxicity levels and lack of patient selection with high levels of tumor hypoxia [20]. A more potent hypoxia-activated prodrug currently undergoing early clinical testing is $\mathrm{TH}-302$. It is a 2nitroimidazole conjugated to bromo-isophosphoramide mustard, which is released and activated upon very low levels of oxygen [21] and diffuses 
to surrounding cells creating a cytotoxic bystander effect [19]. TH-302 displayed clinical activity when used as single agent, which makes it unique compared to earlier generation hypoxia-activated cytotoxins which demonstrate anti-tumor activity only when used in combination with radiation or chemotherapy [22]. Furthermore, TH-302 efficacy was correlated with the hypoxic fraction across different tumor models [2326]. Phase 1 trials have proved TH-302 safety with nausea, vomiting and fatigue as the most frequently occurring toxicities. Other trials successfully combined TH-302 with doxorubicin in patients with advanced soft tissue sarcoma [27] or with gemcitabine in patients with advanced pancreatic cancer [28]. A phase 3 double-blind, placebo-controlled trial has been initiated in which patients with advanced pancreatic cancer were randomized to gemcitabine combined with $\mathrm{TH}-302$ or placebo [29]. Recently our group has evaluated the efficacy of TH-302 in a rat rhabdomyosarcoma and a human $\mathrm{H} 460$ xenograft model, using growth delay as endpoint. TH-302 in both models significantly inhibited tumor growth and markedly sensitized tumors to radiation. Furthermore, the therapeutic effect of TH-302 was depended on the tumor oxygenation status prior to local radiotherapy that was modified by either carbogen (to improve oxygenation) or low oxygen containing gas (to increase hypoxia) breathing [30].

Increasing tumor oxygenation has shown potential for improving radiotherapy efficacy in several randomized clinical trials [31,32]. In spite of positive results, these strategies using hyperbaric oxygen or carbogen combined with vasodilating agents have not gained clinical traction due to practical limitations, toxicity and relatively modest clinical benefit [33]. An alternative strategy to achieve improved tumor oxygenation is to decrease cellular oxygen consumption using for example metformin, an inhibitor of the mitochondrial NADH dehydrogenase, also known as complex 1, activity in the mitochondrial electron transport respiration chain [34]. Recently, it has been demonstrated that metformin increases tumor response to radiotherapy, through a reduction in oxygen consumption and improved tumor oxygenation [35]. For future personalized cancer medicine, evaluation of hypoxia biomarkers and patient stratification will be essential to apply hypoxia targeting treatments to change radiotherapy response.

\section{Hypoxia molecular response}

As tumors progress from an early to later stage disease, supply of oxygen becomes limited. Cancer cells must therefore alter their metabolism to an anaerobic glycolytic phenotype, resulting in a less efficient energy 
production and intracellular acidosis. In order to survive cancer cells must adapt to this acidic microenvironment, which helps promote metastases [36]. One of the important molecular responses to hypoxia is the stabilization of the hypoxia inducible factor (HIF)- $1 \alpha$, enabling interaction with HIF-1 $\beta$. The complex translocates to the nucleus where it binds to hypoxia responsive elements (HRE) in the promoter region of target genes, such as vascular endothelial growth factor (VEGF), glucose transporters 1 (GLUT-1) and carbonic anhydrase IX (CAIX) [37]. CAIX as well as other membrane transporters, like the sodium-proton exchanger 1 (NHE-1) and the monocarboxylate transporters (MCT), are upregulated to counteract the hypoxia-induced intracellular acidosis. CAIX is a tumor specific dimeric membrane bound zinc metallo-enzyme, which catalyses the reversible hydration of carbon dioxide to bicarbonate and a proton to help maintain the cells $\mathrm{pH}$ homeostasis [38]. High tumoral CAIX expression has been associated with poor prognosis, tumor progression and aggressiveness [39]. Inhibition of its function would therefore be a promising anticancer approach to target the hypoxic compartment of tumors.

\section{CAIX imaging tracers}

A molecular imaging approach based on selective ligands to accessible proteins overexpressed at sites of hypoxia is desired. Such an agent could help physicians to decide which patients would benefit from adjuvant hypoxia-targeted therapy, e.g. anti-CAIX therapy. One strategy is using antibodies or antibody fragments targeted against transmembrane CAIX expression. The highly specific antibody M75 recognizes the extracellular proteoglycan-like domain of CAIX and is used for Western blotting and immunohistochemistry [40]. Specific accumulation of iodine-125 radiolabelled derivative has been observed in HT29 tumor-bearing mice [41]. Independently, the monoclonal G250 antibody was developed as a biomarker for renal cell carcinoma [42]. A chimeric version of G250 (cG250) has been radiolabelled with iodine-124, zirconium-89 or indium111 for diagnostic purposes [43]. However, no apparent correlation has been observed between cG250 uptake and pimonidazole labelling or CAIX staining in head and neck tumor models attributed to the large interval between cG250 injection and immunohistochemical evaluation [44]. Pepsin degradation of intact cG250 antibodies resulted in $F\left(a b^{\prime}\right)_{2}$ fragments with a smaller molecular weight resulting in faster clearance from blood and healthy tissues. Zirconium-89 labelled cG250-F(ab') fragments were found to spatially correlate with CAIX expression [45]. 
Furthermore, fully human CAIX single-chain variable fragment (scFv) minibodies have been generated using phage-display technology. They recognize the extracellular carbonic anhydrase domain, but do not inhibit CAIX activity and do not bind to the other transmembrane enzyme CAXII [46]. Recently, near-infrared (NIR) fluorescent monoclonal antibodies against CAIX and CAXII have been successfully tested for the non-invasive detection of breast cancer metastasis [47]. A dual labelled antibody combining indium-11 or iodine-125 nuclear with NIR imaging has proven feasible for preoperative and intraoperative detection of CAIX expressing renal cell carcinomas $[48,49]$.

A second approach is the use of small molecules specifically targeting the active site of CAIX. Several classes of small molecules with low (nM) affinity have been extensively described, but due to the high degree of homology among CA isotypes, small molecules are generally not specific for one isoform [50]. To prevent interaction with the intracellular CA isoforms charged species or bulky groups such as FITC, albumin or sugar moieties are added to the small molecule. These strategies prevent transportation across membranes, but do not guarantee selectivity between CAIX and CAXII, both transmembrane enzymes with their catalytic domains oriented extracellularly. Attempts to design specific compounds targeting each isoform separately have been recently reviewed [51].

We and others have demonstrated in vitro that small molecule binding requires not only CAIX expression but also its hypoxic activation [52,53]. This offers a big advantage compared with antibodies against CAIX, since these small molecules can distinguish cells that are currently hypoxic from those that were previously hypoxic, while antibodies do not since their long half-life after reoxygenation [54]. In vivo, we have reported significant accumulation of fluorescent sulfonamides in HT29 xenografts, which was causally related with tumor oxygenation. Furthermore, bound sulfonamide decreased rapidly upon tumor reoxygenation [55]. Similar results have been obtained using fluorescent acetazolamide derivatives showing preferential targeting of CAIX overexpressing SK-RC-52 renal cell xenografts [56]. Recently, a series of sulfonamide derivatives conjugated with NIR fluorescent dyes having up to 50-fold higher selectivity for CAIX compared to the intracellular and other transmembrane isoforms has been designed. High tumoral uptake with little accumulation in other organs, except for the kidneys, has been observed using fluorescence molecular tomography [57]. Several synthesis strategies have been proposed to enable nuclear imaging, however most attempts were not successful in showing specific enhanced tumor uptake. We have 
synthesized and evaluated a technecium-99m labelled sulfonamide for visualization of CAIX expression by SPECT imaging. Despite favourable affinity values maximum tumor uptake was low $(<0.5 \% \mathrm{ID} / \mathrm{g})$ even after varying physicochemical properties of the molecules $[58,59]$. A range of sulfonamides conjugated metal complexes have recently been designed for metallic radionuclide imaging. Although high in vitro uptake was observed, cellular binding between CAIX positive and negative cell lines was not obviously different [60]. Besides metal chelation approaches, direct fluorine-18 radiolabelling of CAIX targeted molecules has been assessed. Several compounds have been synthetized, such as 7-(2fluoroethoxy)coumarin (FEC) and U-104 [61], the tertiary sulfonamides 4a-c [62] and VM4-037A [63]. Although all derivatives showed good affinity for CAIX with excellent plasma stability, uptake in HT-29 xenografts was minimal which precludes their application as CAIX imaging agents.

\section{CAIX targeting}

Inhibiting CAIX can be done either by the use of monoclonal antibodies or with small molecule inhibitors. Antibody approaches are mostly based on the concept of antibody dependent cell cytotoxicity (ADCC). A leading example is cG250, marketed as RENCAREX ${ }^{\circledR}$, which is extensively investigated as an anticancer immunotherapy [64]. Phase 1 and 2 trials have demonstrated safety and efficacy as monotherapy or in combination with interferon (IFN)- $\alpha$ for the treatment of renal cell carcinoma (RCC) [65]. This antibody was also tested in the double-blind, placebocontrolled phase 3 aRISER trial for adjuvant therapy of clear cell RCC, but as announced by WILEX AG the antibody failed to meet the primary endpoint, since no improvement in median disease-free survival was observed compared to placebo. Several new antibodies currently tested in preclinical settings show promising results regarding anti-tumor effects $[66,67]$.

Specific inhibition of different carbonic anhydrase isoforms using small molecules is an active field of research and has been extensively reviewed $[50,68,69]$. Membrane-impermeable acetazolamide derivatives [56] and aromatic sulfonamides [70] were able to reduce tumor growth and proliferation. Treatment of mammary-tumor bearing mice with CAIXspecific sulfonamide and glycosylcoumarin inhibitors resulted in a significant reduction in tumor growth and lung metastasis formation [71]. One of the several potent CAIX inhibitors identified from a DNA-encoded chemical library screen has shown high and specific accumulation in tumor models [72]. A new class of sulfamate inhibitors proved to be 
excellent candidates for low dosage anti-metastatic drugs [73], but were ineffective in reducing primary tumor growth $[73,74]$. Combining small molecules targeting CAIX with conventional therapies might yield even better efficacy. Recently, a CAIX dependent sensitizing effect of indanesulfonamides [75] and acetazolamide [76] on respectively radioand chemotherapy has been demonstrated. Similarly combination of paclitaxel with oral administered U-104 significantly affected primary tumor growth and metastasis formation by reducing the breast cancer stem cell population [77]. Nitroimidazole and sulfamide based dual targeting drugs reduced hypoxic extracellular acidification in vitro, inhibited tumor growth at low dosage and sensitized tumors to both radiation [78] and doxorubicin [79]. This dual-targeting strategy appeared to be more effective than single targeting molecules. Recently, a family of novel small-molecule drug conjugates comprising of a linker cleavable in the extracellular space and a potent cytotoxic payload targeting CAIX has been designed and characterized. The disulfide-linked conjugate with maytansinoid DM1 as cytotoxic payload and an acetazolamide derivative as the targeting ligand has shown potent anti-tumor effects in renal cell carcinoma models with only minimal toxicity [80]. These results indicate that targeted delivery of potent cytotoxic agents using CAIX directed ligands may provide therapeutic benefits over current standard of care. The first clinical trial (NCT02215850) testing a small molecule CAIX inhibitor, named SLC-0111, is currently ongoing and is focused on testing the safety in subjects with advanced solid tumors.

\section{Window-of-opportunity trial}

Although there are a high number of new promising anti-cancer agents under preclinical and clinical investigation, the success rate of approved drugs for clinical practice has not been significantly increased. Improved clinical trial designs, such as 'window-of-opportunity' trials will help to select effective drugs at an earlier stage and to identify patients who potentially will benefit of the drug. In this trial, the patient agrees to delay combined conventional anti-cancer therapy to first receive the experimental drug, with the aim to obtain knowledge about anti-tumor activity in a disease state that is not disturbed by previous or simultaneous treatments [81]. The question has been raised whether these trials should be more widely applied in early phases of drug development knowing the progress in imaging and monitoring tumor progression [82] to prevent expensive long-lasting classical clinical testing of inefficacious drugs. Using this trial approach, hypoxia imaging can be used as a biomarker of response, especially suitable in the context of testing hypoxia (response) targeting drugs. Upon patient inclusion, 
baseline hypoxia should be acquired followed by the experimental targeting drug. A post-treatment hypoxia PET scan will assess the effect of the single treatment by comparison of the hypoxic fractions between the two scans (Figure 1). This window-of-opportunity trial can precede a phase 1 trial testing safety of the experimental drug in combination with conventional treatment, e.g. radiotherapy, or a randomized phase 2 clinical trial. We have used this concept preclinically to proof efficacy of the hypoxia-activated cytotoxic prodrug $\mathrm{TH}-302$. The hypoxic fraction assessed with HX4 PET imaging in the rhabdomyosarcoma model was significantly reduced at day 4 upon TH-302 treatment, while vehicle treatment was ineffective. Additionally, $\mathrm{TH}-302$ was not only effective as monotherapy, but also sensitized tumors to a single dose of radiation [30]. Similarly, BAY 87-2243, an inhibitor of mitochondrial complex 1 , resulted in reduced HIF-1 $\alpha$ activity and pimonidazole binding prior to radiotherapy improving local tumor control $[83,84]$. Its efficacy has also been shown by a dramatic reduction in FAZA PET signal before significant changes in tumor volume were observed [85]. Finally sunitinib treatment resulted in improved tumor oxygenation as FAZA uptake in Caki-1 renal cell xenografts [86] and in patients with soft-tissue sarcomas [87] was significantly reduced during therapy. Upon withdrawal of sunitinib therapy, FAZA uptake increased again, indicating a rebound in tumor hypoxia. These examples clearly highlight the importance of imaging the hypoxic fraction of tumors to monitor treatment response. 
Figure 1:

Window-of-opportunity trial concept implementing non-invasive hypoxia imaging before and after drug administration followed by phase 1 or 2 trial combining radiotherapy and experimental drug. Additional hypoxia imaging can be included in step 3 for early response monitoring. HX4 hypoxia PET-CT images from a patient with head and neck cancer are shown as proof of concept.

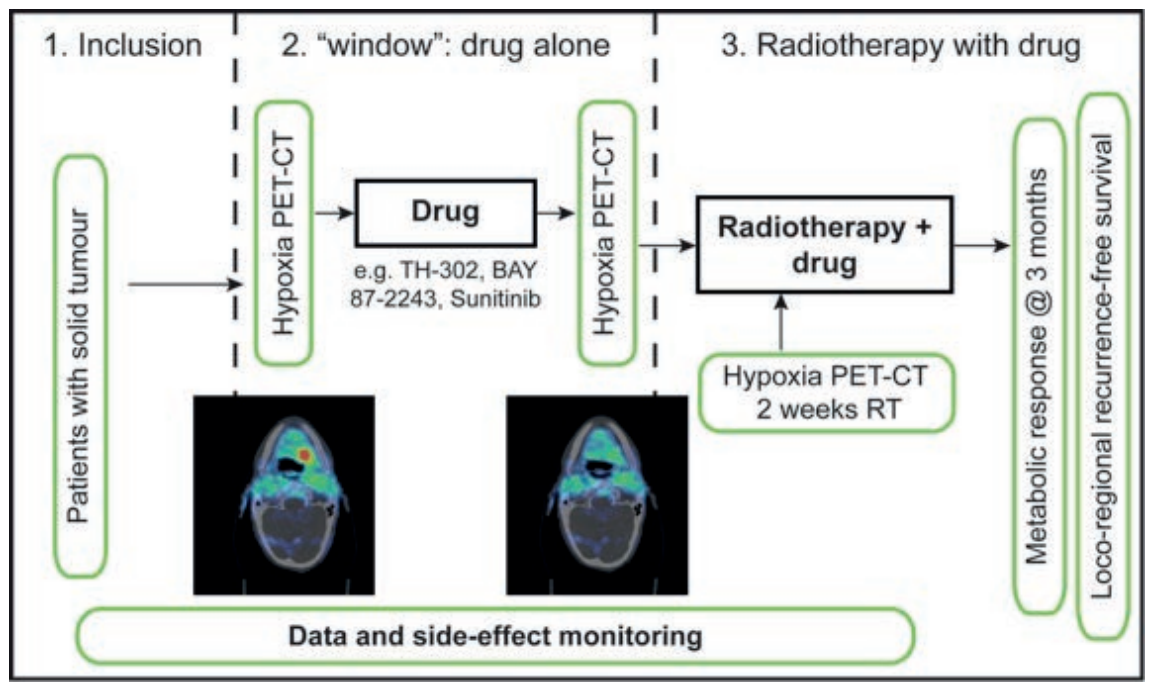




\section{Conclusions}

Current clinically available hypoxia PET tracers, although showing different characteristics, have proven to be reliable for evaluation of tumor hypoxia. Much progress has been made in the synthesis and evaluation of high affinity small molecules targeting CAIX. Nevertheless, proper clinically-suited diagnostic tools are still lacking. The window-ofopportunity trial concept implementing non-invasive imaging to monitor treatment response is an important tool to provide evidence of antitumor efficacy in earlier stages of drug development.

\section{Acknowledgements}

Authors acknowledge financial support from the QulC-ConCePT project, which is partly funded by EFPI A companies and the Innovative Medicine Initiative Joint Undertaking (IMI JU) under Grant Agreement No. 115151. Authors also acknowledge financial support from the EU 7th framework program (METOXIA, EURECA, ARTFORCE, REQUITE), NGI Pre-Seed grant $\left(n^{\circ}\right.$ 93612005), Worldwide Cancer Research grant ( $\left.n^{\circ} 15-0345\right)$, La Ligue contre le Cancer (comité des Pyrénées-Orientales), Kankeronderzoekfonds Limburg from the Health Foundation Limburg and the Dutch Cancer Society (KWF UM 2011-5020, KWF UM 2012-5394, KWF UM 2015-7635, KWF MAC 2013-6089 and KWF MAC 2013-6425). 


\section{References}

[1] Hanahan D, Weinberg RA. Hallmarks of cancer: the next generation. Cell 2011;144:646-74.

[2] Nordsmark M, Bentzen SM, Rudat V, et al. Prognostic value of tumor oxygenation in 397 head and neck tumors after primary radiation therapy. An international multi-center study. Radiother Oncol 2005;77:18-24.

[3] Horsman MR, Mortensen LS, Petersen JB, Busk M, Overgaard J. Imaging hypoxia to improve radiotherapy outcome. Nat Rev Clin Oncol 2012;9:674-87.

[4] Peeters SG, Zegers CM, Yaromina A, Van Elmpt W, Dubois L, Lambin P. Current preclinical and clinical applications of hypoxia PET imaging using 2nitroimidazoles. Q J Nucl Med Mol Imaging 2015;59:39-57.

[5] Rasey JS, Koh WJ, Evans ML, et al. Quantifying regional hypoxia in human tumors with positron emission tomography of [18F]fluoromisonidazole: a pretherapy study of 37 patients. Int J Radiat Oncol Biol Phys 1996;36:417-28.

[6] Krohn KA, Link JM, Mason RP. Molecular imaging of hypoxia. J Nucl Med 2008;49 Suppl 2:129S-48S.

[7] Lopci E, Grassi I, Chiti A, et al. PET radiopharmaceuticals for imaging of tumor hypoxia: a review of the evidence. Am J Nucl Med Mol Imaging 2014;4:365-84.

[8] Kolb HC, Finn MG, Sharpless KB. Click Chemistry: Diverse Chemical Function from a Few Good Reactions. Angew Chem Int Ed Engl 2001;40:2004-21.

[9] Doss M, Zhang JJ, Belanger MJ, et al. Biodistribution and radiation dosimetry of the hypoxia marker 18F-HX4 in monkeys and humans determined by using whole-body PET/CT. Nucl Med Commun 2010;31:1016-24.

[10] Carlin S, Zhang H, Reese M, Ramos NN, Chen Q, Ricketts SA. A comparison of the imaging characteristics and microregional distribution of 4 hypoxia PET tracers. J Nucl Med 2014;55:515-21.

[11] Dubois LJ, Lieuwes NG, Janssen MH, et al. Preclinical evaluation and validation of [18F]HX4, a promising hypoxia marker for PET imaging. Proc Natl Acad Sci U S A 2011;108:14620-5.

[12] van Loon J, Janssen MH, Ollers M, et al. PET imaging of hypoxia using [18F]HX4: a phase I trial. Eur J Nucl Med Mol Imaging 2010;37:1663-8.

[13] Zegers CM, van Elmpt W, Wierts R, et al. Hypoxia imaging with [(1)(8)F]HX4 PET in NSCLC patients: defining optimal imaging parameters. Radiother Oncol 2013;109:58-64.

[14] Zegers CM, van Elmpt W, Reymen B, et al. In vivo quantification of hypoxic and metabolic status of NSCLC tumors using [18F]HX4 and [18F]FDG-PET/CT imaging. Clin Cancer Res 2014;20:6389-97.

[15] Zegers CM, van Elmpt W, Hoebers FJ, et al. Imaging of tumor hypoxia and metabolism in patients with head and neck squamous cell carcinoma. Acta oncologica 2015:1-7.

[16] Peeters SG, Zegers CM, Lieuwes NG, et al. A comparative study of the hypoxia PET tracers [(1)(8)F]HX4, [(1)(8)F]FAZA, and [(1)(8)F]FMISO in a preclinical tumor model. Int J Radiat Oncol Biol Phys 2015;91:351-9.

[17] Chen L, Zhang Z, Kolb HC, Walsh JC, Zhang J, Guan Y. (1)(8)F-HX4 hypoxia imaging with PET/CT in head and neck cancer: a comparison with (1)(8)F-FMISO. Nucl Med Commun 2012;33:1096-102.

[18] Wack LJ, Monnich D, van Elmpt W, et al. Comparison of [18F]-FMISO, [18F]-FAZA and [18F]-HX4 for PET imaging of hypoxia - a simulation study. Acta oncologica 2015:1-8.

[19] Wilson WR, Hay MP. Targeting hypoxia in cancer therapy. Nat Rev Cancer 2011;11:393-410. 
[20] Reddy SB, Williamson SK. Tirapazamine: a novel agent targeting hypoxic tumor cells. Expert Opin Investig Drugs 2009;18:77-87.

[21] Meng F, Evans JW, Bhupathi D, et al. Molecular and cellular pharmacology of the hypoxia-activated prodrug TH-302. Mol Cancer Ther 2012;11:740-51.

[22] Bennewith KL, Dedhar S. Targeting hypoxic tumor cells to overcome metastasis. BMC cancer 2011;11:504.

[23] Cardenas-Rodriguez J, Li Y, Galons JP, et al. Imaging biomarkers to monitor response to the hypoxia-activated prodrug TH-302 in the MiaPaCa2 flank xenograft model. Magn Reson Imaging 2012;30:1002-9.

[24] Hu J, Van Valckenborgh E, Dehui X, et al. Synergistic induction of apoptosis in multiple myeloma cells by bortezomib and hypoxia-activated prodrug $\mathrm{TH}-302$, in vivo and in vitro. Mol Cancer Ther 2013.

[25] Liu Q, Sun JD, Wang J, et al. TH-302, a hypoxia-activated prodrug with broad in vivo preclinical combination therapy efficacy: optimization of dosing regimens and schedules. Cancer Chemother Pharmacol 2012;69:1487-98.

[26] Sun JD, Liu $Q$, Wang J, et al. Selective tumor hypoxia targeting by hypoxiaactivated prodrug TH-302 inhibits tumor growth in preclinical models of cancer. Clin Cancer Res 2012;18:758-70.

[27] Chawla SP, Cranmer LD, Van Tine BA, et al. Phase II study of the safety and antitumor activity of the hypoxia-activated prodrug TH-302 in combination with doxorubicin in patients with advanced soft tissue sarcoma. J Clin Oncol 2014;32:3299-306.

[28] Borad MJ, Reddy SG, Bahary N, et al. Randomized Phase II Trial of Gemcitabine Plus TH-302 Versus Gemcitabine in Patients With Advanced Pancreatic Cancer. J Clin Oncol 2015;33:1475-81.

[29] Van Cutsem E, Fram R, Schlichting M, Ryan D. P-0173 * Phase 3 trial of gemcitabine and $\mathrm{TH}-302$ compared with gemcitabine and placebo in patients with pancreatic adenocarcinoma: The MAESTRO Trial. Ann Oncol 2013;24:iv38iv121.

[30] Peeters SG, Zegers CM, Biemans R, et al. TH-302 in Combination with Radiotherapy Enhances the Therapeutic Outcome and Is Associated with Pretreatment [18F]HX4 Hypoxia PET Imaging. Clin Cancer Res 2015.

[31] Janssens GO, Rademakers SE, Terhaard CH, et al. Accelerated radiotherapy with carbogen and nicotinamide for laryngeal cancer: results of a phase III randomized trial. J Clin Oncol 2012;30:1777-83.

[32] Overgaard J. Hypoxic modification of radiotherapy in squamous cell carcinoma of the head and neck--a systematic review and meta-analysis. Radiother Oncol 2011;100:22-32.

[33] Overgaard J. Hypoxic radiosensitization: adored and ignored. J Clin Oncol 2007;25:4066-74.

[34] van Gisbergen MW, Voets AM, Starmans MH, et al. How do changes in the mtDNA and mitochondrial dysfunction influence cancer and cancer therapy? Challenges, opportunities and models. Mutat Res Rev Mutat Res 2015;764:16-30.

[35] Zannella VE, Dal Pra A, Muaddi $H$, et al. Reprogramming metabolism with metformin improves tumor oxygenation and radiotherapy response. Clin Cancer Res 2013;19:6741-50.

[36] Gatenby RA, Gillies RJ. Why do cancers have high aerobic glycolysis? Nat Rev Cancer 2004;4:891-9.

[37] Wouters BG, Koritzinsky M. Hypoxia signalling through mTOR and the unfolded protein response in cancer. Nat Rev Cancer 2008;8:851-64.

[38] Alterio V, Hilvo M, Di Fiore A, et al. Crystal structure of the catalytic domain of the tumor-associated human carbonic anhydrase IX. Proc Natl Acad Sci U S A 2009;106:16233-8. 
[39] Potter CP, Harris AL. Diagnostic, prognostic and therapeutic implications of carbonic anhydrases in cancer. Br J Cancer 2003;89:2-7.

[40] Pastorekova S, Zavadova Z, Kostal M, Babusikova O, Zavada J. A novel quasi-viral agent, MaTu, is a two-component system. Virology 1992;187:620-6.

[41] Chrastina A, Zavada J, Parkkila S, et al. Biodistribution and pharmacokinetics of 125I-labeled monoclonal antibody M75 specific for carbonic anhydrase IX, an intrinsic marker of hypoxia, in nude mice xenografted with human colorectal carcinoma. Int J Cancer 2003;105:873-81.

[42] Oosterwijk E, Ruiter DJ, Hoedemaeker PJ, et al. Monoclonal antibody G 250 recognizes a determinant present in renal-cell carcinoma and absent from normal kidney. Int J Cancer 1986;38:489-94.

[43] Cheal SM, Punzalan B, Doran MG, et al. Pairwise comparison of 89Zr- and 124Ilabeled cG250 based on positron emission tomography imaging and nonlinear immunokinetic modeling: in vivo carbonic anhydrase IX receptor binding and internalization in mouse xenografts of clear-cell renal cell carcinoma. Eur J Nucl Med Mol Imaging 2014;41:985-94.

[44] Troost EG, Bussink J, Kaanders JH, et al. Comparison of different methods of CAIX quantification in relation to hypoxia in three human head and neck tumor lines. Radiother Oncol 2005;76:194-9.

[45] Hoeben BA, Kaanders JH, Franssen GM, et al. PET of hypoxia with 89Zr-labeled cG250-F(ab')2 in head and neck tumors. J Nucl Med 2010;51:1076-83.

[46] Ahlskog JK, Schliemann C, Marlind J, et al. Human monoclonal antibodies targeting carbonic anhydrase IX for the molecular imaging of hypoxic regions in solid tumors. Br J Cancer 2009;101:645-57.

[47] Tafreshi NK, Bui MM, Bishop K, et al. Noninvasive detection of breast cancer lymph node metastasis using carbonic anhydrases IX and XII targeted imaging probes. Clin Cancer Res 2012;18:207-19.

[48] Muselaers CH, Rijpkema M, Bos DL, et al. Radionuclide and Fluorescence Imaging of Clear Cell Renal Cell Carcinoma Using Dual Labeled Anti-Carbonic Anhydrase IX Antibody G250. J Urol 2015.

[49] Muselaers CH, Stillebroer AB, Rijpkema M, et al. Optical Imaging of Renal Cell Carcinoma with Anti-Carbonic Anhydrase IX Monoclonal Antibody Girentuximab. J Nucl Med 2014;55:1035-40.

[50] Supuran CT. Carbonic anhydrases: novel therapeutic applications for inhibitors and activators. Nat Rev Drug Discov 2008;7:168-81.

[51] Alterio V, Di Fiore A, D'Ambrosio K, Supuran CT, De Simone G. Multiple binding modes of inhibitors to carbonic anhydrases: how to design specific drugs targeting 15 different isoforms? Chem Rev 2012;112:4421-68.

[52] Dubois L, Douma K, Supuran CT, et al. Imaging the hypoxia surrogate marker CA IX requires expression and catalytic activity for binding fluorescent sulfonamide inhibitors. Radiother Oncol 2007;83:367-73.

[53] Svastova E, Hulikova A, Rafajova $M$, et al. Hypoxia activates the capacity of tumor-associated carbonic anhydrase IX to acidify extracellular pH. FEBS Lett 2004;577:439-45.

[54] Rafajova M, Zatovicova M, Kettmann R, Pastorek J, Pastorekova S. Induction by hypoxia combined with low glucose or low bicarbonate and high posttranslational stability upon reoxygenation contribute to carbonic anhydrase IX expression in cancer cells. Int J Oncol 2004;24:995-1004.

[55] Dubois L, Lieuwes NG, Maresca A, et al. Imaging of CA IX with fluorescent labelled sulfonamides distinguishes hypoxic and (re)-oxygenated cells in a xenograft tumor model. Radiother Oncol 2009;92:423-8. 
[56] Ahlskog JK, Dumelin CE, Trussel S, Marlind J, Neri D. In vivo targeting of tumorassociated carbonic anhydrases using acetazolamide derivatives. Bioorg Med Chem Lett 2009;19:4851-6.

[57] Groves K, Bao B, Zhang J, et al. Synthesis and evaluation of near-infrared fluorescent sulfonamide derivatives for imaging of hypoxia-induced carbonic anhydrase IX expression in tumors. Bioorg Med Chem Lett 2012;22:653-7.

[58] Akurathi V, Dubois L, Celen S, et al. Development and biological evaluation of (9)(9)mTc-sulfonamide derivatives for in vivo visualization of CA IX as surrogate tumor hypoxia markers. Eur J Med Chem 2014;71:374-84.

[59] Akurathi V, Dubois L, Lieuwes NG, et al. Synthesis and biological evaluation of a $99 \mathrm{mTc}$-labelled sulfonamide conjugate for in vivo visualization of carbonic anhydrase IX expression in tumor hypoxia. Nucl Med Biol 2010;37:557-64.

[60] Dilworth JR, Pascu SI, Waghorn PA, et al. Synthesis of sulfonamide conjugates of $\mathrm{Cu}(\mathrm{II}), \mathrm{Ga}(\mathrm{III}), \operatorname{In}(\mathrm{III}), \operatorname{Re}(\mathrm{V})$ and $\mathrm{Zn}(\mathrm{II})$ complexes: carbonic anhydrase inhibition studies and cellular imaging investigations. Dalton Trans 2015;44:4859-73.

[61] Pan J, Lau J, Mesak F, et al. Synthesis and evaluation of 18F-labeled carbonic anhydrase IX inhibitors for imaging with positron emission tomography. J Enzyme Inhib Med Chem 2014;29:249-55.

[62] Lau J, Pan J, Zhang Z, et al. Synthesis and evaluation of (18)F-labeled tertiary benzenesulfonamides for imaging carbonic anhydrase IX expression in tumors with positron emission tomography. Bioorg Med Chem Lett 2014;24:3064-8.

[63] Peeters SG, Dubois L, Lieuwes NG, et al. [F]VM4-037 MicroPET Imaging and Biodistribution of Two In Vivo CAIX-Expressing Tumor Models. Mol Imaging Biol 2015.

[64] Surfus JE, Hank JA, Oosterwijk E, et al. Anti-renal-cell carcinoma chimeric antibody G250 facilitates antibody-dependent cellular cytotoxicity with in vitro and in vivo interleukin-2-activated effectors. J Immunother Emphasis Tumor Immunol 1996;19:184-91.

[65] Siebels $M$, Rohrmann $K$, Oberneder R, et al. A clinical phase $1 /$ II trial with the monoclonal antibody cG250 (RENCAREX(R)) and interferon-alpha-2a in metastatic renal cell carcinoma patients. World J Urol 2011;29:121-6.

[66] Birkhauser FD, Koya RC, Neufeld C, et al. Dendritic cell-based immunotherapy in prevention and treatment of renal cell carcinoma: efficacy, safety, and activity of Ad-GM.CAIX in immunocompetent mouse models. J Immunother 2013;36:10211.

[67] Chang DK, Moniz RJ, Xu Z, et al. Human anti-CAIX antibodies mediate immune cell inhibition of renal cell carcinoma in vitro and in a humanized mouse model in vivo. Mol Cancer 2015;14:119.

[68] McDonald PC, Winum JY, Supuran CT, Dedhar S. Recent developments in targeting carbonic anhydrase IX for cancer therapeutics. Oncotarget 2012;3:8497.

[69] Neri D, Supuran CT. Interfering with $\mathrm{pH}$ regulation in tumors as a therapeutic strategy. Nat Rev Drug Discov 2011;10:767-77.

[70] Cianchi F, Vinci MC, Supuran CT, et al. Selective inhibition of carbonic anhydrase IX decreases cell proliferation and induces ceramide-mediated apoptosis in human cancer cells. J Pharmacol Exp Ther 2010;334:710-9.

[71] Lou Y, McDonald PC, Oloumi A, et al. Targeting tumor hypoxia: suppression of breast tumor growth and metastasis by novel carbonic anhydrase IX inhibitors. Cancer Res 2011;71:3364-76.

[72] Buller F, Mannocci L, Zhang Y, Dumelin CE, Scheuermann J, Neri D. Design and synthesis of a novel DNA-encoded chemical library using Diels-Alder cycloadditions. Bioorg Med Chem Lett 2008;18:5926-31. 
[73] Gieling RG, Babur M, Mamnani L, et al. Antimetastatic effect of sulfamate carbonic anhydrase IX inhibitors in breast carcinoma xenografts. J Med Chem 2012;55:5591-600.

[74] Meijer TW, Bussink J, Zatovicova M, et al. Tumor microenvironmental changes induced by the sulfamate carbonic anhydrase IX inhibitor S4 in a laryngeal tumor model. PLoS One 2014;9:e108068.

[75] Dubois L, Peeters S, Lieuwes NG, et al. Specific inhibition of carbonic anhydrase IX activity enhances the in vivo therapeutic effect of tumor irradiation. Radiother Oncol 2011;99:424-31.

[76] Gieling RG, Parker CA, De Costa LA, et al. Inhibition of carbonic anhydrase activity modifies the toxicity of doxorubicin and melphalan in tumor cells in vitro. J Enzyme Inhib Med Chem 2013;28:360-9.

[77] Lock FE, McDonald PC, Lou Y, et al. Targeting carbonic anhydrase IX depletes breast cancer stem cells within the hypoxic niche. Oncogene 2013;32:5210-9.

[78] Dubois L, Peeters SG, van Kuijk SJ, et al. Targeting carbonic anhydrase IX by nitroimidazole based sulfamides enhances the therapeutic effect of tumor irradiation: a new concept of dual targeting drugs. Radiother Oncol 2013;108:523-8.

[79] Rami M, Dubois L, Parvathaneni NK, et al. Hypoxia-targeting carbonic anhydrase IX inhibitors by a new series of nitroimidazolesulfonamides/sulfamides/sulfamates. J Med Chem 2013;56:8512-20.

[80] Krall N, Pretto F, Decurtins W, Bernardes GJ, Supuran CT, Neri D. A smallmolecule drug conjugate for the treatment of carbonic anhydrase IX expressing tumors. Angew Chem Int Ed Engl 2014;53:4231-5.

[81] Orloff J, Douglas F, Pinheiro J, et al. The future of drug development: advancing clinical trial design. Nat Rev Drug Discov 2009;8:949-57.

[82] Glimelius B, Lahn M. Window-of-opportunity trials to evaluate clinical activity of new molecular entities in oncology. Ann Oncol 2011;22:1717-25.

[83] Helbig L, Koi L, Bruchner K, et al. BAY 87-2243, a novel inhibitor of hypoxiainduced gene activation, improves local tumor control after fractionated irradiation in a schedule-dependent manner in head and neck human xenografts. Radiation oncology 2014;9:207.

[84] Helbig L, Koi L, Bruchner K, et al. Hypoxia-inducible factor pathway inhibition resolves tumor hypoxia and improves local tumor control after single-dose irradiation. International journal of radiation oncology, biology, physics 2014;88:159-66.

[85] Chang E, Liu H, Unterschemmann K, et al. 18F-FAZA PET imaging response tracks the reoxygenation of tumors in mice upon treatment with the mitochondrial complex I inhibitor BAY 87-2243. Clin Cancer Res 2015;21:335-46.

[86] Chapman DW, Jans HS, Ma I, et al. Detecting functional changes with [(18)F]FAZA in a renal cell carcinoma mouse model following sunitinib therapy. Eur J Nucl Med Mol Imaging 2014;4:27.

[87] Lewin J, Khamly KK, Young RJ, et al. A phase Ib/II translational study of sunitinib with neoadjuvant radiotherapy in soft-tissue sarcoma. $\mathrm{Br} J$ Cancer 2014;111:2254-61. 


\section{Chapter 3}

\section{PET imaging with the ${ }^{89} \mathrm{Zr}$ labeled EGFR antibody, Cetuximab}

Manuscript in preparation

Panth KM*, Peeters SGJA*, Lieuwes NG, Yaromina A, Poot AJ, Bussink J, Van Dongen GAMS, Dubois LJ, Lambin $\mathrm{P}$

* Equal contribution 


\section{Abstract}

Objective: Treatment with Cetuximab, Epidermal Growth Factor Receptor (EGFR) antibody has not been successful in all patients despite of prior selection for EGFR expression. We hypothesize that this discrepancy between EGFR status and cetuximab accumulation in the tumor could be caused by differences in tumor micro-environmental characteristics.

Material and Methods: Head and neck squamous cell carcinoma primary xenograft lines (SCCNij202, SCCNij82 and SCCNij3) were implanted in nude mice. Upon an average tumor volume of 294 (range 45-2524) $\mathrm{mm}^{3}$ mice were injected with $\left[{ }^{89} \mathrm{Zr}\right]$-cetuximab $(3.6 \pm 1.3 \mathrm{MBq})$. microPET imaging was performed at 24,48 and 72 hours post injection (h p.i.) followed by tumor excision for histological evaluation of EGFR and phosphorylated AKT (pAKT) expression and tumor micro-environmental parameters.

Results: EGFR expression was highly present in SCCNij82 tumors (35.8 $\pm 9.0 \%)$ followed by SCCNij202 $(22.3 \pm 10.6 \%)$ and SCCNij3 (6.6 $\pm 4.8 \%)$ xenografts. microPET imaging with $\left[{ }^{89} \mathrm{Zr}\right]$-cetuximab at $72 \mathrm{~h}$ p.i. showed high mean standardized uptake values $\left(S_{U V} V_{\text {mean }}\right)$ for SCCNij202 $(1.2 \pm 0.4)$ and SCCNij3 $(1.0 \pm 0.3)$ while SCCNij82 with the highest EGFR expression had a significantly lower $\operatorname{SUV}_{\text {mean }}(0.6 \pm 0.3 ; \mathrm{P}<0.05)$. PAKT was quantified as measure for downstream EGFR signaling and found to be higher in SCCNij202 $(7.0 \pm 4.7 \%)$ compared to SCCNij82 $(4.1 \pm 3.5 \%)$ and SCCNij3 $(2.0$ $\pm 1.6 \% ; P=0.025$ ). Pimonidazole hypoxic fraction was significantly lower in SCCNij82 compared to the other tumor models $(\mathrm{P}<0.001)$ and did correlate with the mean TBR determined by microPET. Furthermore, the relative vessel area (RVA) correlated with the mean TBR and was similar in SCCNij202 $(1.9 \pm 1.4 \%)$ and SCCNij82 $(1.0 \pm 0.6 \%)$ while SCCNij3 had a higher RVA $(3.5 \pm 1.1 \%)$.

Conclusion: This study indicates that the observed discrepancy between EGFR protein expression and ${ }^{89} \mathrm{Zr}$-cetuximab uptake in the tumor in the three investigated primary tumor models might be influenced by the amount of hypoxia and the relative vessel area. 


\section{Introduction}

Epidermal Growth Factor Receptor (EGFR) is a transmembrane protein belonging to the ErbB family of receptor tyrosine kinases. This protein is frequently overexpressed in tumors and its high expression is associated with a more aggressive tumor phenotype and higher resistance towards anticancer therapies [1, 2]. EGFR signaling occurs upon ligand binding to the extracellular domain resulting in dimerization of two receptors accompanied by activation of the intracellular domain and downstream signaling pathways like RAS/ERK and PI3K/AKT. These pathways alter proliferation, differentiation and survival [3], important processes involved in the evolvement of cancer [4].

Several treatments have been developed to specifically target EGFR. Tyrosine Kinase Inhibitors (TKI) of EGFR are effective drugs although resistance is acquired over time [5]. Inhibitors specifically targeting the $\mathrm{PI3K} / \mathrm{AKT} / \mathrm{mTOR}$ pathway are being explored as monotherapy or in combination therapies to improve the antitumor effect [6]. Furthermore, monoclonal antibodies targeting EGFR are used in combination with conventional anticancer therapies [7].

Cetuximab is a monoclonal antibody (mAb) specifically targeting and inhibiting the function of EGFR. By preventing the ligand binding to the receptor, dimerization and subsequent activation of the downstream targets is blocked [8]. However, not all patients benefit from this antiEGFR therapy. This may, at least in part, be explained by mutations in the downstream pathway of EGFR, which make the tumors insensitive to the cetuximab treatment [9]. Furthermore, the lack of treatment response might be explained by low availability of the mAb in the tumor. Labeling cetuximab with radioactive tracers like Zirconium-89 $\left({ }^{89} \mathrm{Zr}\right)$ or Copper-64 $\left({ }^{64} \mathrm{Cu}\right)$ for positron emission tomography (PET) imaging can be used to monitor the distribution of this mAb throughout the body and specifically in the tumor. In head and neck squamous cell carcinomas a discrepancy was observed between $\left[{ }^{64} \mathrm{Cu}\right]$-DOTA-cetuximab uptake in the tumor and EGFR expression [10]. Furthermore, a discrepancy between EGFR expression and $\left[{ }^{89} \mathrm{Zr}\right]$-cetuximab was observed in a panel of xenograft tumors [11]. These results indicate that there are factors other than EGFR expression influencing the availability or uptake of cetuximab in the tumor.

In this study we hypothesize that differences in the tumor microenvironment can influence the cetuximab uptake in the tumor. To assess this we used three primary head and neck squamous cell 
carcinoma (HNSCC) xenograft and U373 glioblastoma tumor models which differ in EGFR expression and tumor micro-environmental characteristics. We report microPET and biodistribution data for the $\left.{ }^{89} \mathrm{Zr}\right]$-cetuximab uptake supported by immunohistochemistry staining of the tumor micro-environment. 


\section{Materials \& Methods}

Synthesis of ${ }^{89} \mathrm{Zr}$-labeled cetuximab

$\left[{ }^{89} \mathrm{Zr}\right.$ ] was produced as previously described [12]. [89Zr] labeling of the mAb cetuximab was described by Perk et al. [13]. The control antibody Retuximab was labeled to $\left[{ }^{89} \mathrm{Zr}\right]$ via similar synthesis.

\section{Animal and tumor model}

All animal experimental procedures were approved by the Animal Ethical Committee of Maastricht University. Head and neck squamous cell carcinoma (HNSCC) xenografts were grown and passaged by transplanting $1 \mathrm{~mm}^{3}$ pieces of viable tumor subcutaneously in the lateral flank of NMRI$n u(n u / n u)$ mice. The U373FIpIn/EGFRwt glioblastoma cells, containing a doxycycline inducible EGFRwt overexpressing construct, were cultured as described previously [14]. Tumor cells resuspended in Basement Membrane Matrix (Matrigel ${ }^{\mathrm{TM}}$ BD Biosciences; $3 \times 10^{6}$ cells in 50 ul) were injected in the lateral flank of NMRI-nu (nu/nu) mice. Half of the established tumors were exposed to doxycycline, but the expression of EGFR and other microenvironmental characteristics were not modified (Supplementary Fig 1). Tumor volume was calculated by using the formula $A \times B \times C \times \pi / 6$ in which $A, B$ and $C$ are the three orthogonal diameters of the tumor, as measured using a Vernier caliper, each corrected for the thickness of the skin. On an average volume of 426 (range 45-2524) $\mathrm{mm}^{3}$ for the xenograft tumors and 184 (range 53-303) $\mathrm{mm}^{3}$ for the U373 tumors mice were injected with $\left[{ }^{89} \mathrm{Zr}\right.$-labeled cetuximab (3.6 $\pm 1.3 \mathrm{MBq}$; cetuximab dose $\approx 3.8 \mathrm{mg} / \mathrm{kg}$ ) via the lateral tail vein. $\left[{ }^{89} \mathrm{Zr}\right.$ ]-rituximab imaging was performed only in the U373 model (3.0 $\pm 0.4 \mathrm{MBq}$ ). Food and water was available ad libitum for the duration of the study. Isoflurane inhalation anesthesia (4\% induction, $1-2 \%$ maintenance in $100 \% \mathrm{O}_{2}$ at a flow rate of $1.5 \mathrm{~L} / \mathrm{min}$ ) was used during all animal experiments. One hour and one minute prior to euthanization animals were respectively injected with pimonidazole $(60 \mathrm{mg} / \mathrm{kg}$, i.p.) for hypoxia evaluation and Hoechst 33342 (15 mg/kg i.v.) for assessment of perfusion.

\section{microPET imaging and acquisition}

PET imaging was performed using the Focus 120 microPET (Siemens Medical Solutions USA, Inc) with an axial field of view (FOV) of $7.6 \mathrm{~cm}$, a transaxial FOV of $10 \mathrm{~cm}$ and a resolution of approximately $1.5 \mathrm{~mm}$. After tracer injection, microPET imaging was performed at three time points: 24, 48 and 72 hours post injection. Positioning of the animal was done by placing the cross section of the tumor in the center of the detector ring. A 
25 minute emission scan was obtained that was corrected for random counts, dead time and decay. List mode acquisition and reconstruction were performed as previously described [11]. Image visualization was done by ASIPro VM software (version 6.3.3.0) and analyses were performed by manually drawing three-dimensional regions of interest (ROIs) of the tumor, muscle of the hind leg and heart (blood). From the voxels within the ROls, the mean and maximum activity data (in $\mathrm{Bq} / \mathrm{ml}$ ) were obtained. Data were corrected for decay towards time point of injection. Standardized uptake values (SUV) and tumor to background ratios were calculated for each time point of imaging.

\section{Biodistribution}

After the last image acquisition animals were sacrificed under anesthesia and dissected for biodistribution. Tumor, blood, skin, bladder, heart, lungs, liver, spleen, stomach, small intestine, large intestine, kidneys, salivary glands, muscle and brain were weighed and the amount of radioactivity in each tissue was assessed in a gamma counter. Half of the tumor was used for gamma counting, the other half was snap frozen for histological examination. Disintegrations for each sample were collected over 1 minute, corrected for decay, cross-calibrated to the microPET and the percentage injected dose per gram of tissue was calculated.

Immunofluorescence and quantification

The sections $(5 \mu \mathrm{m})$ were cut from the central part of frozen tumor material from both U373 and xenograft tumors. In a first experiment from which the staining were performed at the Radboud University in Nijmegen (Netherlands) U373 tumors were stained for EGFR, hypoxia (pimonidazole) and vessels. Sections were fixed with cold aceton, scanned for perfusion (Hoechst signal), rehydrated in phosphate buffered saline (PBS) and preincubated with normal donkey serum before they were exposed to the primary antibody for EGFR (1:50; goat, Santa Cruz Biotochnology, CA, USA) and pimonidazole (1:2000; rabbit, gift from J.A. Raleigh University of North Carolina) and vessels. Secondary antibody was added after washing in PBS, followed by staining for vessels (1:100; 9F1 supernatant, a chicken monoclonal antibody against rat endothelium, was a gift from the Department of Pathology, Radboud University Nijmegen Medical Centre) and nuclei (Hoechst). Secondary antibodies used were donkey anti goat Alexa568 (1:600), donkey anti rabbit Alexa488 (1:600) and chicken anti rat Alexa 647 (1:100) (Molecular Probes). After staining, sections were scanned and quantified as described previously [15]. 
In a second experiment xenograft tumors were stained at Maastricht University (Netherlands) for hypoxia (pimonidazole), vessels (CD31), EGFR or pAKT. After fixation in cold aceton, hydrating in TBS-tween and incubating in normal goat serum, primary antibody was incubated: rabbit anti-pimonidazole (1:150, HP3-1000, Bio-connect), rat anti-mouse CD31 (1:500 ,BD biosciences), rabbit polyclonal anti-EGFR (1:50 Santa Cruz Biotechnology, Inc) or rabbit polyclonal anti-phosphor-AKT (Ser473) (1:25 Cell Signaling). Wash with TBS-tween and incubate with secondary antibody: goat anti rabbit Alexa594 (1:500), goat anti rat Alexa488 (1:750), both Invitrogen). After washing with TBS-tween and mounting with fluorescent mounting medium (DakoCytomation), images were acquired using an Olympus BX51WI microscope equipped with a Hamamatsu EM-CCD C9100 digital camera, a motorized stage (Ludl Mac 2000) and a 10x objective. Micromanager 1.4 software was used for automated image acquisition [16]. Stitching of images and quantitative analyses were performed using ImageJ version $1.49 \mathrm{e}$ (http://rsb.info.nih.gov/ij/). All image recordings and analyses were performed by two investigators blinded to the subject coding. Non-viable and non-tumor tissue were excluded from the analysis by manual delineation using information from the same sections stained for hematoxylin and eosin after image acquisition. To discriminate signal from background, threshold setting was manually applied. The stainings were quantified as percent of positively stained area in viable tumor area.

\section{Statistics}

All statistics were performed and graphs were made in GraphPad Prism 5. To determine statistically significant differences between two independent groups, a t test was used. Correlations were calculated by performing a linear regression analysis with a Pearson test. Probability value of less than 0.05 was considered significant. 


\section{Results}

The tumor uptake of Zirconium-89 labeled cetuximab specific to EGFR was assessed in three HNSCC xenograft models, SCCNij202, SCCNij3 and SCCNij82 using microPET imaging. High uptake was observed in the tumor and the liver area (representative images Figure $1 \mathrm{~A}$ ). Quantification of the tumor region showed a slightly increasing signal over time with the highest SUV at $72 \mathrm{~h}$ p.i. (Figure 1B). Both SCCNij202 and SCCNij3 have a significantly $(P<0.05)$ higher SUV $_{\text {mean }}$ value compared to SCCNij82 at all time-points (Figure 1B). SUV $\max$ for SCCNij202 was significantly higher than for SCCNij82 at both $48 \mathrm{~h}$ p.i. and $72 \mathrm{~h}$ p.i $(\mathrm{P}<0.05)$ and SCCNij3 SUV $\max$ was significantly higher compared to SCCNij82 at $72 \mathrm{~h}$ p.i. $(\mathrm{P}<0.05)$.

Additionally, tumor to background ratios were calculated using both blood and muscle as reference (Figure 1B). Mean tumor to blood (TBR) ratios were significantly higher for SCCNij3 compared to SCCNij82 at both $48 \mathrm{~h}$ p.i. and $72 \mathrm{~h}$ p.i. with an average TBR of $1.5 \pm 0.3$ for SCCNij 3 at $72 \mathrm{~h}$ p.i. For the other tumor to background ratios no significant differences were observed (figure 1B). In U373 tumor bearing animals no significant differences in tumor to background ratios were observed between the EGFR basal (no dox) and the EGFR overexpressing (dox) tumors (Supplement figure $1 \mathrm{~A} \& \mathrm{~B}$ ). Furthermore, the tumor to background ratios of the non-tumor specific tracer $\left[{ }^{89} \mathrm{Zr}\right]$-Rituximab was comparable to $\left[{ }^{89} \mathrm{Zr}\right]$-Cetuximab uptake in that model. Only on mean TBR for doxycycline exposed animals $\left[{ }^{89} \mathrm{Zr}\right.$ ]-Rituximab values were lower than the TBR of $\left[{ }^{89} \mathrm{Zr}\right]$-Cetuximab (Supplement figure 1A\&B).

Biodistribution in individual organs was performed after the last scan at $72 \mathrm{~h}$ p.i. and showed the highest \%ID (injected dose)/gram in SCCNij3 (5.1 $\pm 1.4 \%)$ followed by SCCNij202 (3.7 $\pm 2.0 \%)$, which is significantly lower $(\mathrm{P}<0.05)$, and SCCNij82 $(3.1 \pm 2.2 \%)$. This is comparable with the mean TBR in which SCCNij3 also has the highest ratio and SCCNij82 the lowest (Figure 2A). Besides high uptake in the tumor, uptake above $2.5 \% \mathrm{ID} / \mathrm{g}$ was observed in the lung and in the liver. Uptake in the SCCNij82 tumor model was not statistically different from uptake in the skin while all other organs had a significant lower \%ID/g than all the tumor models. Furthermore no differences between the distribution of $\left[{ }^{89} \mathrm{Zr}\right]$-Cetuximab and $\left[{ }^{89} \mathrm{Zr}\right.$ ]-Rituximab were observed in the biodistribution of the U373 bearing animals (Supplement figure 2A).

Furthermore, characteristics of the tumor micro-environment were studied by immunohistochemical staining (representative images Figure 3A). EGFR expression was significantly higher in both SCCNij202 (22.3 
$\pm 10.6 \%)$ and SCCNij82 (35.8 $\pm 9.0 \%)$ compared to SCCNij3 (6.6 $\pm 4.8 \%$, $\mathrm{P}=0.006$ and $\mathrm{P}=<0.0001$ respectively; Figure $3 \mathrm{~B}$ ) which is in contradiction with the SUV uptake values which are high for SCCNij202 and SCCNij3 while SCCNij82 had low uptake of $\left[{ }^{89} \mathrm{Zr}\right.$ ]-cetuximab. Phosphorylated AKT (pAKT) was quantified as a measure for downstream EGFR signaling and showed the highest expression in SCCNij202 $(7.0 \pm 4.7 \%)$ followed by SCCNij82 $(4.1 \pm 3.5 \%)$. The relative PAKT area for SCCNij3 was the lowest with $2.0 \pm 1.6 \%$ and was significantly lower compared to SCCNij202 $(P=0.03)$ and corresponds with the EGFR expression of this tumor model. Furthermore the hypoxic fraction as determined by pimonidazole staining, was higher in both SCCNij202 $(25.6 \pm 6.5)$ and SCCNij3 $(25.0 \pm 6.2)$ compared to SCCNij82 (8.6 $\pm 3.2 ; \mathrm{P}<0.001$ for both). Relative vessel area was the highest in the high TBR tumor model SCCNij3 $(3.5 \pm 1.1 \%)$, followed by SCCNij202 (1.8 $\pm 1.4 \%)$ and the low TBR tumor model SCCNij82 (1.0 $\pm 0.6 \%)$.

Staining of the U373 tumor microenvironment revealed almost no expression of EGFR regardless of their exposure to doxycycline or not to induce the EGFR expression ( $1.5 \pm 2.1 \%$ for no dox animals $1.2 \pm 0.7 \%$ for dox animals), an average hypoxic fraction of $42.5 \pm 16.9 \%$, an average perfusion fraction of $21.6 \pm 13.4 \%$ and an average vessel fraction of 6.3 $\pm 3.6 \%$ (Supplement figure $3 \mathrm{~A}$ ). For xenograft models a positive correlation was found between the mean tumor to blood ratio and the hypoxic fraction $\left(R^{2}=0.34, P=0.02\right)$ and relative vessel area $\left(R V A ; R^{2}=0.38\right.$, $\mathrm{P}=0.009$ ). 


\section{Discussion}

The aim of this study was to investigate which microenvironmental characteristics influence cetuximab uptake in the tumors. The uptake of $\left[{ }^{89} \mathrm{Zr}\right]$-cetuximab was determined in three HNSCC xenograft models with different EGFR expression using microPET imaging and biodistribution. Furthermore, tumor micro-environmental characteristics were studied to assess differences in hypoxia, pAKT status and relative vessel area.

Differences in SUV were detected between the different tumor models, with SCCNij202 and SCCNij3 having the highest SUV and tumor to background ratios. Biodistribution did reveal the highest tumor uptake values for the SCCNij3 model. $\left[{ }^{89} \mathrm{Zr}\right.$ ]-cetuximab was initially highly present in the blood and was cleared over time. This can be observed in the TBR which increases over time while the SUV of the tumor is stable. The initial uptake in the muscle was much lower and almost no clearance occurred in this background tissue demonstrated in the stable TMR. This is confirmed in the biodistribution where the muscle shows much lower uptake than the blood fraction. Furthermore, biodistribution indicates liver as high uptake organ for $\left[{ }^{89} \mathrm{Zr}\right]$-cetuximab or its derived catabolites, which can also be observed in the PET images. This suggests that clearance of ${ }^{89} \mathrm{Zr}$ ]-cetuximab occurs via the liver. The uptake in the lung has a large spread possibly due to contamination of the un-rinsed tissue with blood cloths originating from the heart punction, causing higher values than expected. Although in patients skin toxicity is an often observed side effect of cetuximab treatment [17], this is not observed or expected in this study since the imaging dose used here is much lower than a therapeutic dose. Furthermore, cetuximab is a humanized antibody and therefore does not cross react with mouse skin.

EGFR expression in stained tumor sections revealed the highest expression in the SCCNij82 model and the lowest in the SCCNij3 model. This is in discrepancy with the $\left[{ }^{89} \mathrm{Zr}\right]$-cetuximab tumor uptake as quantified by SUV or TBR (Figure 1,3) which is consistent with the findings of others [10,11]. However, when EGFR expression is very low no $\left[{ }^{89} \mathrm{Zr}\right.$ ]-cetuximab accumulates in the tumor (supplement figure 1, 2, 3) which was shown before in a MDA-MB-435 [18] and a T-47D [11] EGFR negative tumor model indicating that EGFR should be expressed on tumor cells for cetuximab uptake.

Furthermore a correlation between the mean TBR and the HF was observed as well as a correlation between the mean TBR and the relative vessel area. The correlation was only observed using mean TBR indicating 
that this tumor to background ratio holds different information than the SUV. When the analysis were restricted per tumor model, the correlation between mean TBR and relative vessel area holds true for 2 out of 3 models. This suggests that the distribution of $\left[{ }^{89} \mathrm{Zr}\right]$-cetuximab is mainly dependent on the blood supply to the tumor. A study using $\left[{ }^{64} \mathrm{Cu}\right]$-DOTApanitumumab, a mAb targeting EGFR comparable to cetuximab, in HNSCC tumor models also suggested that the tumor uptake was dependent on the vessel density and the vascular permeability [19]. Additionally they suggest that the high affinity of the mAbs to the receptors might cause a binding-site barrier [19], meaning that the mAbs bind to the first receptor they encounter thereby retarding antibody percolation [20]. The results from our study are in line with this reasoning showing that the tumor model with low $\left[{ }^{89} \mathrm{Zr}\right]$-cetuximab uptake has the lowest relative vessel area while EGFR expression is high. In contrast the tumor model with the highest $\left[{ }^{89} \mathrm{Zr}\right]$-cetuximab uptake has the highest relative vessel area, although EGFR expression in this tumor is low, clearly demonstrating that cetuximab uptake does not depend on the extent of EGFR expression. In addition it is well known that the blood vessel development in tumors is often impaired and consist of chaotic structured, leaky vessels [21]. This complex microvasculature can limit the antibody delivery to the tumor [22]. Furthermore, the relatively large size of antibodies might be limited for the homogeneous distribution throughout the body and even be worse in poor vascularized tumors. In general it is presumed that tumors with low vascular density have less uptake of anticancer agents compared with highly vascularized tumors [23].

Indium(111) labeled cetuximab- $F\left(a b^{\prime}\right)_{2}$ fragments have been developed for SPECT imaging and a good correlation with tumor EGFR expression has been shown [24, 25]. These $F\left(a b^{\prime}\right)$ fragments consist of only a part of the full size monoclonal antibody cetuximab and therefore have a fast distribution and a rapid clearance from the background [25]. ${ }^{111} \mathrm{In}$ cetuximab- $F\left(a b^{\prime}\right)$ autoradiography does correlate with the distribution of EGFR in the tumor after a single dose of radiotherapy [24]. However, it can be questioned whether ${ }^{111}$ In-cetuximab-F $\left(a b^{\prime}\right)$ does represent the distribution of cetuximab and whether it is predictive for the treatment outcome

In this study cetuximab was used in an imaging dose without any therapeutic intent. Anti-cancer cetuximab treatment prevents the dimerization of EGFR and thereby the activation of downstream pathways like PAKT [8]. In therapeutically sensitive tumors this would cause an inverse correlation between cetuximab and pAKT. However, the PAKT measured in this study only gives an indication of the basal downstream 
EGFR signaling without any interference. Other studies investigating the treatment response of cetuximab indicate that mutations in the downstream pathway of EGFR, e.g. in PTEN or KRAS, can modify processes like cell growth and apoptosis [26, 27].

In clinical setting not all patients benefit from cetuximab treatment [28]. Our data support the findings of others that this first of all can be caused by the poor vascularization and perfusion of the tumor. In addition, mutations in downstream pathways of EGFR can prevent the therapeutic effect of cetuximab [29]. Therefore non-invasive imaging of cetuximab uptake together with the identification of mutations in downstream EGFR pathway may represent a powerful predictive tool to select patients for specific treatments. 


\section{Conclusions}

In conclusion, the uptake of $\left[{ }^{89} \mathrm{Zr}\right]$-cetuximab was independent of EGFR expression in the observed tumor models. Rather, the uptake was influenced by multiple factors as both hypoxia and microvessel density had significant impact on the antibody uptake.

\section{Acknowledgments}

"The micrographs in this paper were taken with a confocal spinning disk microscope financed by The Netherlands Organisation for Scientific Research (NWO), grant number 911-06-003."

KWF Zr-C225 
Figure 1

${ }^{89}$ Zr-cetuximab PET imaging and quantification. A) Representative PET images from xenograft model SCCNij 202, SCCNij 3 and SCCNij 82 at 72h p.i. B) Mean and max values for SUV, tumor to blood and tumor to muscle ratios are represented at time point 24,48 and 72 hours post injection (h p.i.) for SCCNij $202(n=6)$, SCCNij $3(n=8)$ and SCCNij $82(n=6)$ tumor models. Data represent the mean + SD and $* P<0.05$.

A
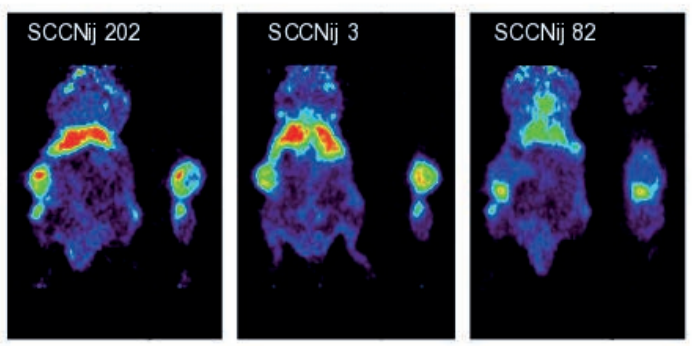

B
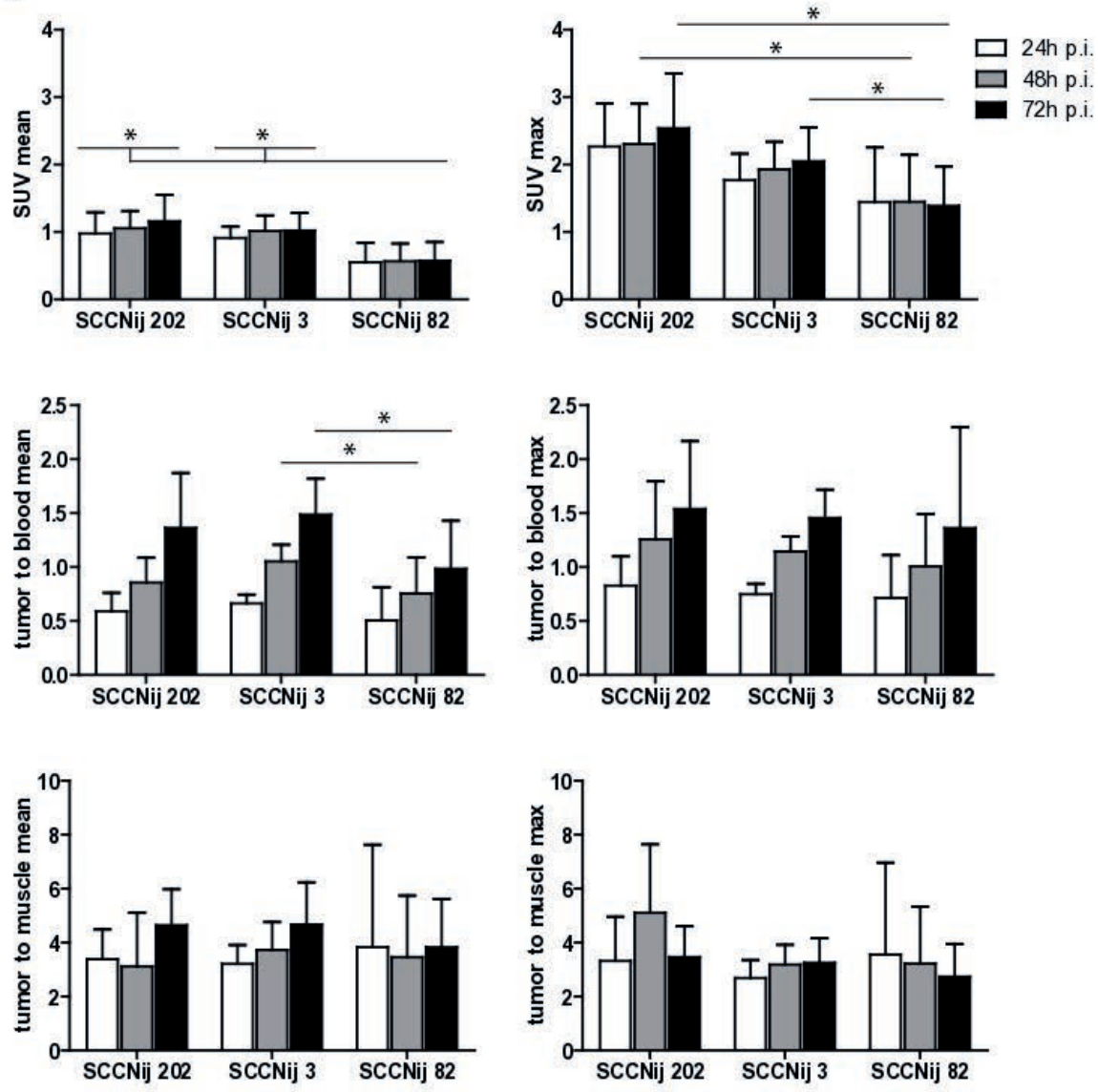
Figure 2

Biodistribution of ${ }^{89} \mathrm{Zr}$-cetuximab A) Biodistribution was performed $72 \mathrm{~h}$ p.i. on the three tumor models SCCNij $202(n=6)$, SCCNij $3(n=8)$ and SCCNij $82(n=7)$ and organs $(n=16)$. Data represent the mean + SD for all organs. ${ }^{*} P<0.05$.

A

Biodistribution

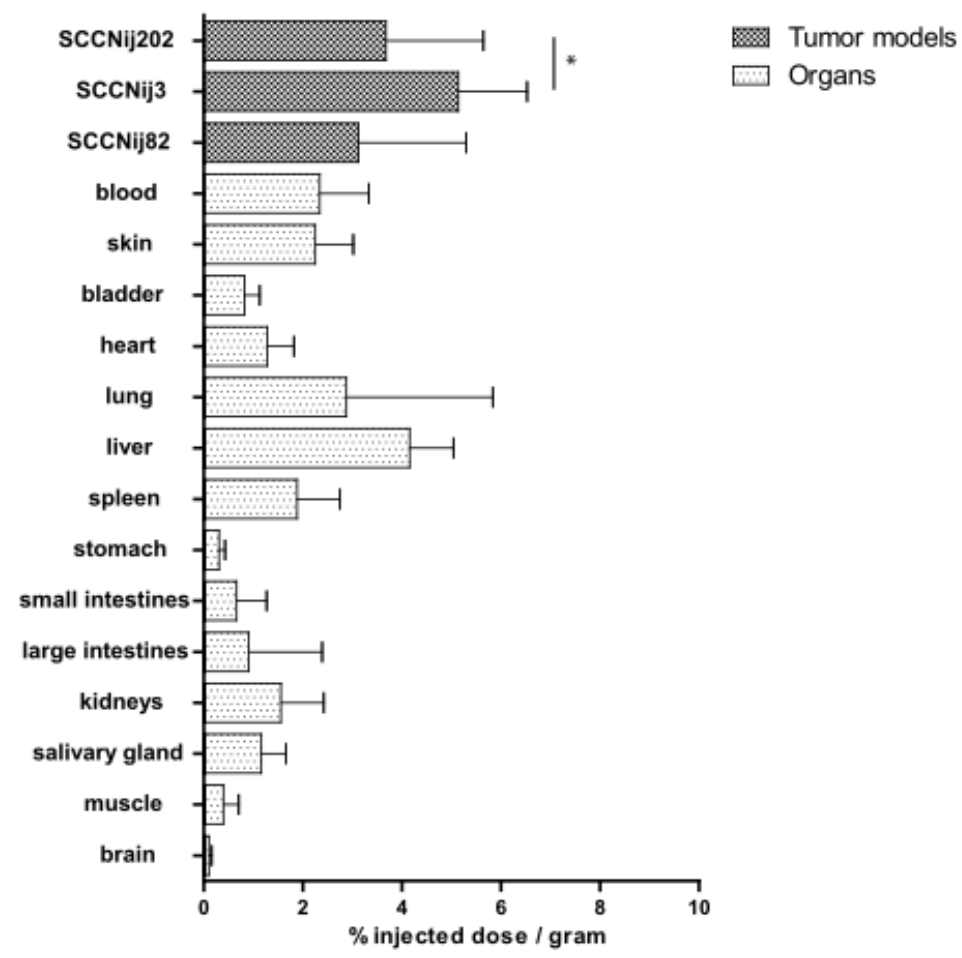


Figure 3

Tumor micro-environmental characteristics A) representative image of a SCCNij 202 tumor stained for EGFR (magnate), p-AKT (blue) and merged hypoxia (Pimo; green) and vessels (CD31; red). B) Quantification of the stainings for the three tumor models SCCNij $202(n=5)$, SCCNij $3(n=7)$ and SCCNij $82(n=5)$. All stainings are represented as a percentage of the viable tumor tissue. Data represent mean +SD. $* P<0.05, * * P<0.01, * * * P<0.001$, $* * * * \mathrm{P}<0.0001$.

\section{A}

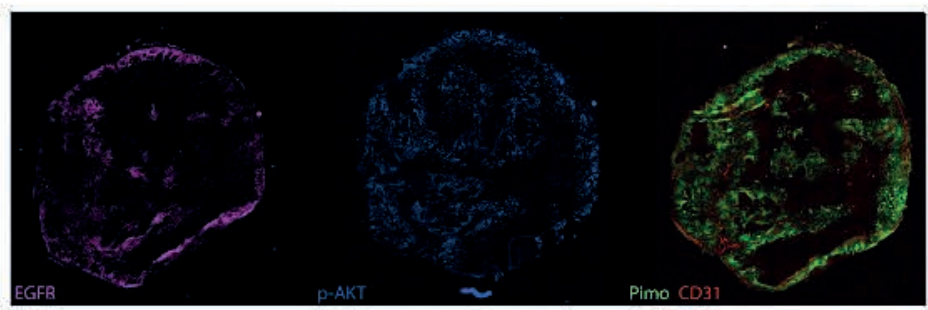

B
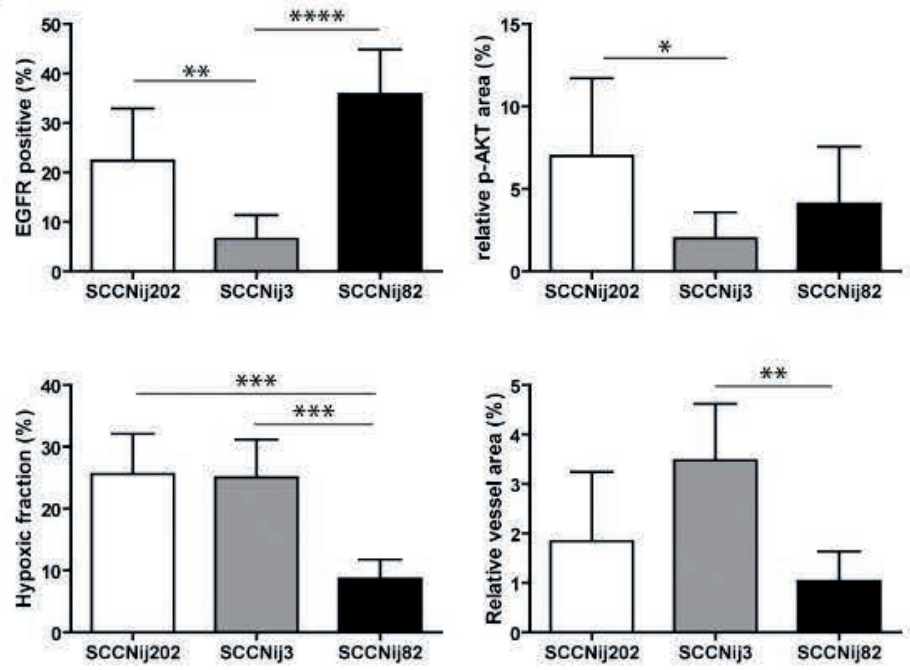

C
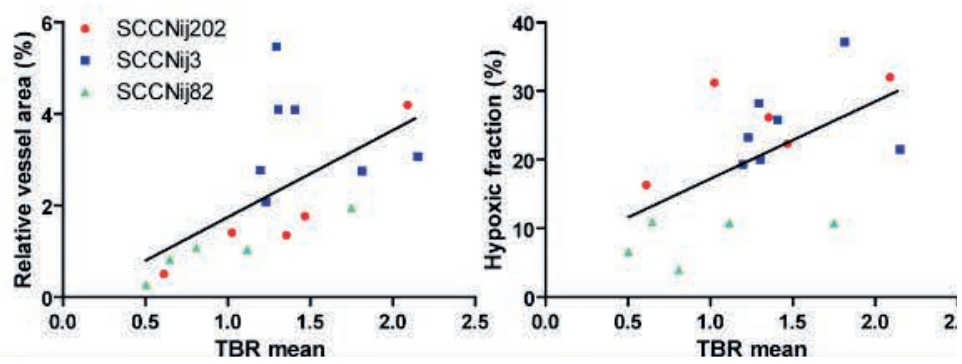


\section{Supplement figure 1}

Quantification of ${ }^{89} \mathrm{Zr}$-cetuximab and ${ }^{89} \mathrm{Zr}$-rituximab uptake in U373 tumors. A) Mean and maximal tumor to blood and tumor to muscle ratios at 24,48 and $72 \mathrm{~h}$ p.i. Data represent the mean +SD. For ${ }^{89} \mathrm{Zr}$-cetuximab no dox $\mathrm{n}=9$ and dox $\mathrm{n}=8$ and ${ }^{89} \mathrm{Zr}$-rituximab $\mathrm{n}=4$. Data represent mean $+S D, * P<0.05$.

A
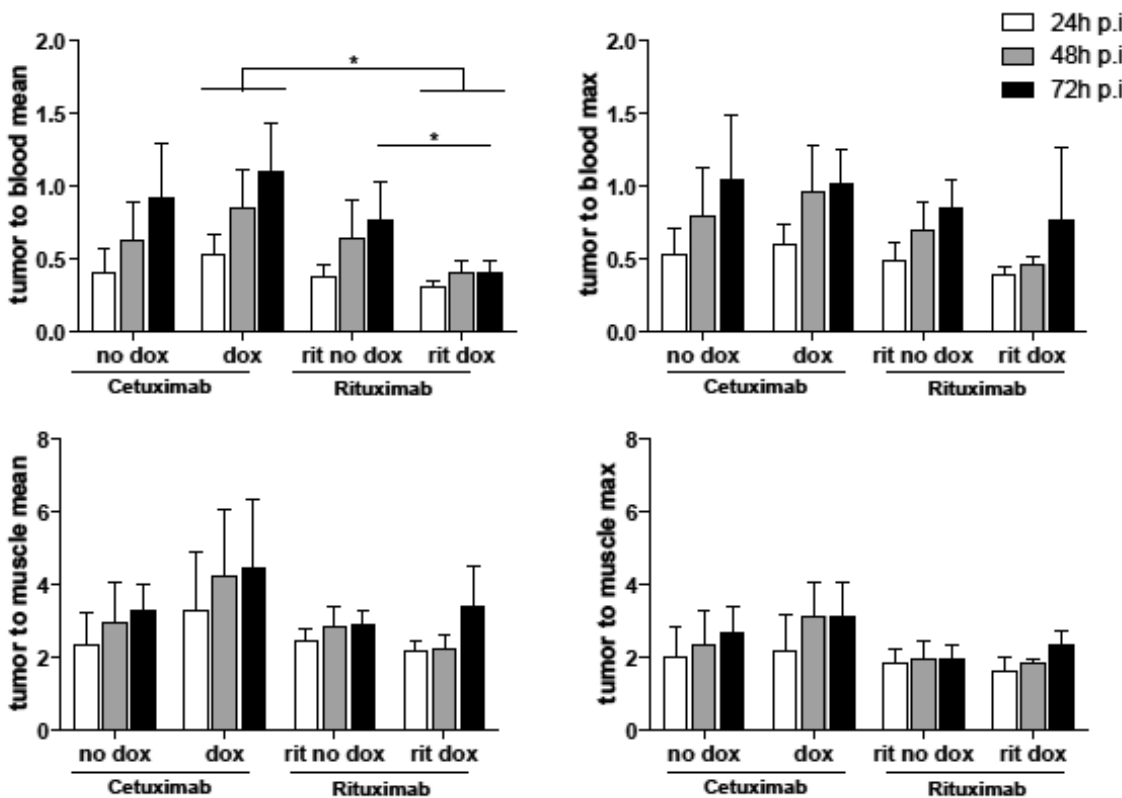


\section{Supplement figure 2}

Biodistribution A) Biodistribution was performed $72 \mathrm{~h}$ p.i. on the U373 model. Data represent the mean + SD for all organs. For $\left[{ }^{89} \mathrm{Zr}\right]$-cetuximab tumor no dox $\mathrm{n}=7$, tumor dox $=9$ and organs $n=16$. For $\left[{ }^{89} Z\right.$ r $]$-rituximab tumor no dox $n=4$, tumor dox $n=4$ and organs $n=8$. Data represent mean $+S D, * P<0.05$.

\section{A}

\section{Cetuximab}

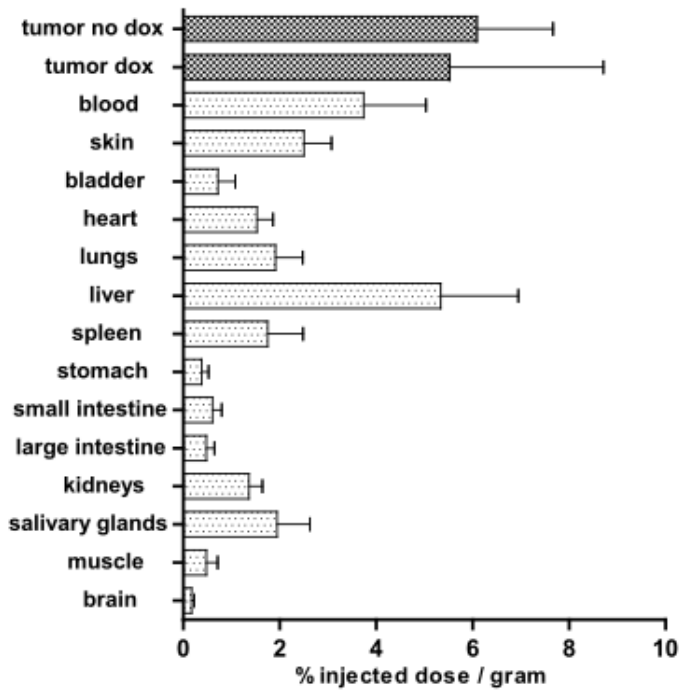

Rituximab

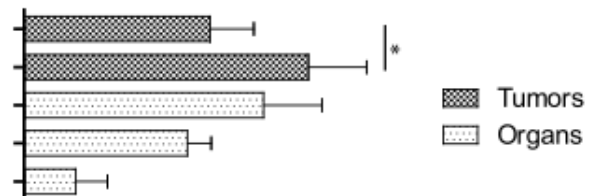

\section{Supplement figure 3}

Tumor micro-environmental characteristics A) Stainings were performed on U373 tumors. Data represent the mean + SD of EGFR no dox $n=8$, dox $n=7$; hypoxia (pimo) no dox $n=8$, dox $n=7$; perfusion (Hoechst) no dox $n=5$, dox $n=4$ and vascular no dox $n=7$, dox $n=7$.

A

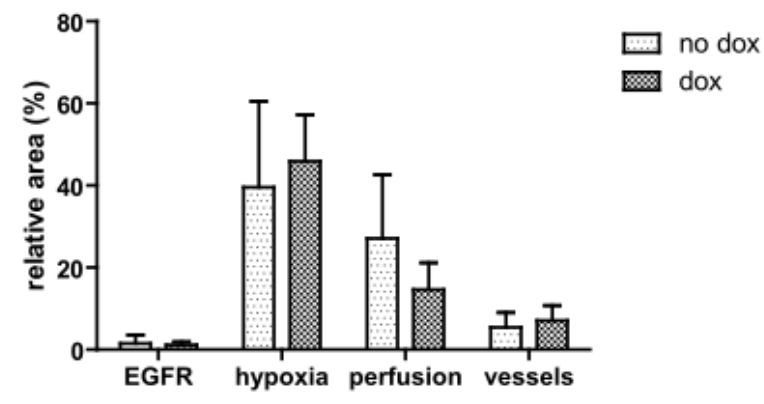




\section{References}

1. Akimoto T, Hunter NR, Buchmiller L, Mason K, Ang KK, Milas L. Inverse relationship between epidermal growth factor receptor expression and radiocurability of murine carcinomas. Clin Cancer Res. 1999; 5: 2884-90.

2. Eriksen JG, Steiniche T, Overgaard J. The influence of epidermal growth factor receptor and tumor differentiation on the response to accelerated radiotherapy of squamous cell carcinomas of the head and neck in the randomized DAHANCA 6 and 7 study. Radiother Oncol. 2005; 74: 93-100.

3. Citri A, Yarden Y. EGF-ERBB signalling: towards the systems level. Nat Rev Mol Cell Biol. 2006; 7: 505-16. doi:10.1038/nrm1962.

4. Hanahan D, Weinberg RA. Hallmarks of cancer: the next generation. Cell. 2011; 144: 646-74. doi:10.1016/j.cell.2011.02.013.

5. Asami K, Atagi S. Epidermal growth factor receptor tyrosine kinase inhibitors for non-small cell lung cancer. World J Clin Oncol. 2014; 5: 646-59. doi:10.5306/wjco.v5.i4.646.

6. Sun Z, Wang Z, Liu X, Wang D. New development of inhibitors targeting the $\mathrm{PI3K} / \mathrm{AKT} / \mathrm{mTOR}$ pathway in personalized treatment of non-small-cell lung cancer. Anticancer Drugs. 2014. doi:10.1097/CAD.0000000000000172.

7. Trivedi S, Concha-Benavente F, Srivastava RM, Jie HB, Gibson SP, Schmitt NC, et al. Immune biomarkers of anti-EGFR monoclonal antibody therapy. Ann Oncol. 2014. doi:10.1093/annonc/mdu156.

8. Mendelsohn J. Targeting the epidermal growth factor receptor for cancer therapy. J Clin Oncol. 2002; 20: 1S-13S.

9. De Stefano A, Carlomagno C. Beyond KRAS: Predictive factors of the efficacy of anti-EGFR monoclonal antibodies in the treatment of metastatic colorectal cancer. World J Gastroenterol. 2014; 20: 9732-43. doi:10.3748/wjg.v20.i29.9732.

10. Niu G, Sun X, Cao Q, Courter D, Koong A, Le QT, et al. Cetuximab-based immunotherapy and radioimmunotherapy of head and neck squamous cell carcinoma. Clin Cancer Res. 2010; 16: 2095-105. doi:10.1158/1078-0432.ccr-092495.

11. Aerts HJ, Dubois L, Perk L, Vermaelen P, van Dongen GA, Wouters BG, et al. Disparity between in vivo EGFR expression and 89Zr-labeled cetuximab uptake assessed with PET. J Nucl Med. 2009; 50: 123-31. doi:10.2967/jnumed.108.054312.

12. Verel I, Visser GW, Boellaard R, Stigter-van Walsum M, Snow GB, van Dongen GA. $89 \mathrm{Zr}$ immuno-PET: comprehensive procedures for the production of $89 \mathrm{Zr}$-labeled monoclonal antibodies. J Nucl Med. 2003; 44: 1271-81.

13. Perk LR, Visser GW, Vosjan MJ, Stigter-van Walsum M, Tijink BM, Leemans CR, et al. (89) Zr as a PET surrogate radioisotope for scouting biodistribution of the therapeutic radiometals (90)Y and (177)Lu in tumor-bearing nude mice after coupling to the internalizing antibody cetuximab. J Nucl Med. 2005; 46: 1898906.

14. Theys J, Jutten B, Dubois L, Rouschop KM, Chiu RK, Li Y, et al. The deletion mutant EGFRvIII significantly contributes to stress resistance typical for the tumour microenvironment. Radiother Oncol. 2009; 92: 399-404. doi:10.1016/j.radonc.2009.06.017.

15. Stegeman H, Kaanders JH, Wheeler DL, van der Kogel AJ, Verheijen MM, Waaijer SJ, et al. Activation of AKT by hypoxia: a potential target for hypoxic tumors of the head and neck. BMC Cancer. 2012; 12: 463. doi:10.1186/1471-2407-12-463.

16. Edelstein A, Amodaj N, Hoover K, Vale R, Stuurman N. Computer control of microscopes using microManager. Curr Protoc Mol Biol. 2010; Chapter 14: Unit14 20. doi:10.1002/0471142727.mb1420s92. 
17. Nechushtan H, Vainer G, Stainberg H, Salmon AY, Hamburger T, Peretz T. A phase $1 / 2$ of a combination of Cetuximab and Taxane for "triple negative" breast cancer patients. Breast. 2014; 23: 435-8. doi:10.1016/j.breast.2014.03.003.

18. Ping Li W, Meyer LA, Capretto DA, Sherman CD, Anderson CJ. Receptor-binding, biodistribution, and metabolism studies of 64CU-DOTA-cetuximab, a PET-imaging agent for epidermal growth-factor receptor-positive tumors. Cancer Biother Radiopharm. 2008; 23: 158-71. doi:10.1089/cbr.2007.0444.

19. Niu G, Li Z, Xie J, Le QT, Chen X. PET of EGFR antibody distribution in head and neck squamous cell carcinoma models. J Nucl Med. 2009; 50: 1116-23. doi:10.2967/jnumed.109.061820.

20. Fujimori K, Covell DG, Fletcher JE, Weinstein JN. A modeling analysis of monoclonal antibody percolation through tumors: a binding-site barrier. J Nucl Med. 1990; 31: 1191-8.

21. Brown JM, Wilson WR. Exploiting tumour hypoxia in cancer treatment. Nat Rev Cancer. 2004; 4: 437-47. doi:10.1038/nrc1367.

22. Penta R, Ambrosi D. The role of the microvascular tortuosity in tumor transport phenomena. J Theor Biol. 2014; 364C: 80-97. doi:10.1016/j.jtbi.2014.08.007.

23. Maeda H. Vascular permeability in cancer and infection as related to macromolecular drug delivery, with emphasis on the EPR effect for tumorselective drug targeting. Proc Jpn Acad Ser B Phys Biol Sci. 2012; 88: 53-71.

24. van Dijk LK, Boerman OC, Franssen GM, Lok J, Kaanders JH, Bussink J. Early response monitoring with 18F-FDG PET and cetuximab-F(ab')2-SPECT after radiotherapy of human head and neck squamous cell carcinomas in a mouse model. J Nucl Med. 2014; 55: 1665-70. doi:10.2967/jnumed.114.141762.

25. van Dijk LK, Hoeben BA, Stegeman H, Kaanders JH, Franssen GM, Boerman OC, et al. 111/n-cetuximab-F(ab')2 SPECT imaging for quantification of accessible epidermal growth factor receptors (EGFR) in HNSCC xenografts. Radiother Oncol. 2013; 108: 484-8. doi:10.1016/j.radonc.2013.06.034.

26. Bouali S, Chretien AS, Ramacci C, Rouyer M, Becuwe P, Merlin JL. PTEN expression controls cellular response to cetuximab by mediating PI3K/AKT and RAS/RAF/MAPK downstream signaling in KRAS wild-type, hormone refractory prostate cancer cells. Oncology reports. 2009; 21: 731-5.

27. Julien S, Merino-Trigo A, Lacroix L, Pocard M, Goere D, Mariani P, et al. Characterization of a large panel of patient-derived tumor xenografts representing the clinical heterogeneity of human colorectal cancer. Clin Cancer Res. 2012; 18: 5314-28. doi:10.1158/1078-0432.CCR-12-0372.

28. Petrelli F, Coinu A, Riboldi V, Borgonovo K, Ghilardi M, Cabiddu M, et al. Concomitant platinum-based chemotherapy or cetuximab with radiotherapy for locally advanced head and neck cancer: A systematic review and meta-analysis of published studies. Oral Oncol. 2014. doi:10.1016/j.oraloncology.2014.08.005.

29. Soeda H, Shimodaira H, Gamoh M, Ando H, Isobe H, Suto T, et al. Phase II trial of cetuximab plus irinotecan for oxaliplatin- and irinotecan-based chemotherapyrefractory patients with advanced and/or metastatic colorectal cancer: evaluation of efficacy and safety based on KRAS mutation status (T-CORE0801). Oncology. 2014; 87: 7-20. doi:10.1159/000360989. 


\section{Chapter 4}

\section{Optical Imaging of Matric Metallo Proteinase 2 (MMP2)}

Published in: Scientific Reports, 2016 Feb, 6, 22198

Panth KM, Van den Beucken T, Biemans R, Lieuwes NG, Weber M, Losen $M$, Yaromina A, Dubois LJ, Lambin P 


\section{Abstract:}

Matrix metalloproteinase-2 (MMP2) is important in tumorigenesis, angiogenesis and tumor invasion. In this study, we investigated if the Cy5tagged small immuno protein targeting the catalytic domain of human MMP2 (aMMP2-SIP) detects MMP2 in tumors non-invasively. For this purpose, we generated MMP2 expressing (empty vector EV) and knockdown (KD) HT1080, U373 and U87 cells, which were injected subcutaneously in the lateral flank of NMRI-nu mice. Optical imaging (Optix MX2) performed at 0.5, 2, 4, 8, 24 and 48 hour post injection (h.p.i.) of Cy5 tagged aMMP2-SIP, indicated significantly lower tumor to background ratios at both $24(P=0.0090)$ and 48 h.p.i. $(P<0.0001)$ for the U87 MMP2-KD compared to control tumors. No differences were found for HT1080 and U373 models. U87 MMP2-KD tumors had significantly lower MMP2 activity $(\mathrm{P}<0.0001)$ than EV tumors as determined by gelatin zymography in tumor sections and lysates, while no differences were observed between EV and MMP2-KD in HT1080 and U373. In line with these data, only U87 MMP2-KD tumors had a reduced tumor growth compared to control tumors $(\mathrm{P}=0.0053)$. aMMP2-SIP uptake correlates with MMP2 activity and might therefore be a potential non-invasive imaging biomarker for the evaluation of MMP2 activity in tumors. 
Introduction: The number of reports on the role of matrix metalloproteinases (MMPs) in cancer progression has tremendously increased over the past years. MMPs play a prominent role in cancer invasion and metastasis to a large degree by disrupting and remodeling of the extracellular matrix (ECM). However, MMPs are also involved in many other important processes during tumorigenesis like proliferation, angiogenesis, apoptosis and migration ${ }^{1-3}$. This raised interest in developing broad-spectrum MMP inhibitors which however failed in subsequent clinical trials because of unspecific targeting related to the extended structure homology of $\mathrm{MMPs}^{4}$. Another possible explanation for the failure of the broad-spectrum MMP inhibitors could be that MMPs are thought to be more important in early tumor development, while patients with early stage cancer were not included in these trials ${ }^{4}$. Furthermore, MMPs are tightly regulated at transcriptional level and can have a protective role in tumorigenesis ${ }^{4,5}$. Therefore, research efforts are directed towards gaining improved insights on the essential MMPs in tumor progression and to target individual MMPs.

MMP2 (gelatinase A) breaks down type IV collagen, gelatin, elastin, proteoglycans and other collagenous compounds of the ECM ${ }^{6}$. It is upregulated in many cancers ${ }^{7}$ like glioblastomas ${ }^{8}$, melanomas ${ }^{9,10}$, breast cancer ${ }^{11}$ and colon cancer ${ }^{12}$. MMP2 plays a vital role in angiogenesis ${ }^{13,14}$ and is overexpressed under hypoxic conditions ${ }^{15}$. MMP2 expression in tumors is known to promote invasion and metastasis ${ }^{16,17}$ which correlates with the worse prognosis and aggressive behavior associated with these tumors. Moreover, MMP2 inhibition has been shown to cause radiosensitization ${ }^{18,19}$, a decrease in tumor growth and invasiveness $13,20,21$. Altogether this evidence identifies MMP2 as an interesting target for the development of both diagnostic and therapeutic approaches. MMP2 imaging can aid in detecting aggressive tumors, might serve as a surrogate marker of invasion or as biomarker for patient selection in MMP inhibitors trials.

Advances in antibody-based imaging have enabled major progress in detecting and treating cancers ${ }^{22,23}$. Antibody-based imaging is sensitive and aids in diagnosis, drug selection, drug development and monitoring treatment efficacy. Imaging strategies using whole IgG antibodies however are limited due to slow antibody clearance from blood ${ }^{24,25}$. To circumvent this disadvantage small antibody fragments (minibodies) have been generated by antibody engineering techniques to have superior clearing rates without losing binding characteristics ${ }^{24-26}$. Small chain variable fragments (ScFV) consist of a heavy and a light chain of the variable domains linked by a peptide. ScFv fragments have very fast 
clearance rates from blood due to small size which is desirable for imaging, but on the other hand, only a small amount of the antibody reaches the tumor ${ }^{27}$. Small immuno protein (SIP) format antibodies have an ScFv fragment linked with the constant domain $\left(\varepsilon_{\mathrm{S}_{2}} \mathrm{CH} 4\right)$ of the human IgE secretory isoform ${ }^{28}$ making them more stable than ScFv fragments. Furthermore, the affinity of SIP is equivalent to full length antibody ${ }^{28}$. Recently an antibody selectively targeting catalytic domain of human MMP2 in small immuno protein (aMMP2-SIP) format has been specifically developed for imaging purposes ${ }^{29,30}$.

In this study, we investigated the potential of aMMP2-SIP to detect MMP2 expression in tumors in a non-invasive way. For this, we performed near infrared fluorescence Imaging (NIRF) using Cy5 labeled aMMP2-SIP in mice bearing genetically engineered xenograft tumors. We have evaluated aMMP2-SIP uptake using MMP2 knock-down models, as negative control in different tumor types with varying MMP2 expression and activity. 


\section{Results:}

\section{aMMP2-SIP uptake does not solely dependent on MMP2 expression}

To select an appropriate model a cell lines panel was first screened for MMP2 mRNA expression by using qPCR (Fig. 1A). Cell lines with high MMP2 expression were further confirmed for MMP2 activity by zymography (Fig. 1B). U87 had the highest MMP2 activity, followed by U20S, HT1080, U373 and Hela. Invasive cell lines HT1080 and U373 with intermediate MMP2 expression and non-invasive U87 with highest MMP2 expression were selected for generation of MMP2-knock-down (KD) models. We silenced MMP2 in HT1080, U373 and in U87 by RNA interference to exclude variation in MMP2 uptake between cell lines that is not attributed to MMP2 expression. The knock-down efficacy was determined at both mRNA and protein levels by using qPCR and western blotting respectively. MMP2 mRNA (Fig.1C), protein expression (Fig. 1D \& $1 \mathrm{E}$ ) and activity (Fig. 1F \& 1G) was significantly reduced in all knock-down cell lines compared to empty vector (EV) bearing controls. Both HT1080 and U373 cell lines had a reduced invasion upon silencing MMP2 (Fig. $1 \mathrm{H})$. Altogether this validates the knock-down models in vitro.

To determine the potential use of aMMP2-SIP as an imaging tracer, control (EV) and MMP2-KD cells of HT1080, U373 and U87 were grown as xenograft tumors in mice. As an additional negative control, HCT116 cells having the lowest MMP2 mRNA expression were grown as xenograft tumors. At an average tumor volume of $294 \pm 187 \mathrm{~mm}^{3}$, NIRF imaging was performed at several time points after injection of 75 ug of aMMP2-SIP. The optimal time point of imaging was determined by analyzing images acquired at time points from 0.5 to 48 hours post injection (Fig. 2A). Tumor and background fluorescent intensities were high at the early time points. However, the background signal cleared fast while specific signal was retained in the tumors. Tumor to background signal ratio (TBR) started to increase $24 \mathrm{~h}$ post injection for most of the tumor models. At 48 h.p.i. the background signal was negligible therefore no further improvement in TBR was expected at later time points. As hypothesized, HCT116 with the lowest MMP2 expression had a low antibody uptake (2.79 \pm 1.33). HT1080 with intermediate MMP2 expression had an intermediate uptake but with a large variation within the group (5.69 \pm 5.04).Surprisingly, for U373 with intermediate MMP2 expression, TBR was found to be very low $(1.86 \pm 0.81)$. U87 with highest MMP2 expression had the highest uptake of all (4.42 \pm 1.99$)$ (Fig. 2B).

Next, we assessed aMMP2-SIP uptake in the MMP2-KD models. 
Surprisingly, antibody uptake was not changed in the HT1080 and U373 MMP2-KD tumors (Fig. 3A and 3B) compared to control. TBR for the U373 models was low ( $1.86 \pm 0.81$ for EV and $1.42 \pm 0.77$ for MMP2-KD) and did not increase with time. Although TBR gradually increased over time in the HT1080 models, no significant $(P=0.49)$ differences were observed between MMP2-KD $(6.76 \pm 3.13)$ and control $(5.69 \pm 5.04)$ tumors. On the other hand, the U87 MMP2-KD tumors $(1.60 \pm 0.70)$ had a significantly lower aMMP2-SIP uptake $(\mathrm{P}<0.0001)$ compared to the control tumors $(4.42 \pm 1.99)$ at optimal time point i.e, 48 h.p.i. (Fig. 3C). Altogether the aMMP2-SIP uptake patterns did not correlate with MMP2 mRNA and protein expression in the xenograft tumors (Supplementary fig. 1)

aMMP2-SIP uptake correlates with MMP2 activity and $\alpha v \beta 3$ expression: Since aMMP2-SIP did not correlate with MMP2 expression levels, we investigated if aMMP2-SIP uptake could be affected by tumor microenvironmental factors like hypoxia, vasculature or perfused vessels (Supplementary fig. 2 and supplementary fig 3A). Percentage of hypoxic fraction determined by quantification of pimonidazole positive areas in the tumors showed no differences between the control and knockdown tumors in all three tumor models (HT1080 P $=0.9504$, U373 P $=0.5366$ and U87 $P=0.1577)$. Percentage of relative vascular area evaluated by CD31 staining also did not significantly differ between the control and knockdown tumors (HT1080 P=0.2009, U373 P=0.1069, and U87 $\mathrm{P}=$ 0.2927). Next, percentage of perfused fraction in the tumors also did not vary between the control and knockdown tumors (HT1080 $P=0.0554$, U373 $\mathrm{P}=0.8173$ and U87 $\mathrm{P}=0.2663$ ). Therefore, hypoxia, vascularization and perfusion did not influence aMMP2-SIP uptake in our models since there were no differences observed between control and MMP2-KD tumors in all three investigated tumor models (Supplementary fig. 3A). Next we evaluated if difference in MMP2 activity could explain discrepancies in aMMP2-SIP uptake. U87 MMP2-KD xenografts had significantly reduced MMP2 activity compared to controls $(P<0.0001)$, while no differences were observed in HT1080 and U373 tumors compared to control tumors (Fig. 4A), despite significantly reduced MMP2 levels (Supplementary Fig. 1). In line with the activity data, only MMP2-KD tumors of U87 had a significantly reduced tumor growth rate compared to control tumors ( $\mathrm{P}=0.0053$ ) (Fig. 5).

Next, we assessed if upstream MMP14 and $\alpha v \beta 3$ expression, previously implicated in MMP2 activity regulation $31,32,33,34$, were affected upon silencing MMP2 expression. As expected, no differences were observed 
between control and MMP2-KD tumors for MMP14 expression (Supplementary fig. 3B \& C). Interestingly, expression of $\alpha \vee \beta 3$ was significantly reduced in MMP2-KD tumors compared to control only in U87 tumors $(P=0.0123)$ in line with MMP2 activity (Fig. 4B). To find further evidence for this association, we assessed the distribution of active MMP2 and $\alpha \vee \beta 3$ on microregional level in U87 tumor cross sections. Regions of high MMP2 activity were observed in areas with overlapping $\alpha \vee \beta 3$ and MMP2 expression (Fig. 4C). Altogether, the data supports that aMMP2-SIP uptake depends on MMP2 activity which was validated in U87 tumors. In line with this finding, neither MMP2 activity nor aMMP2-SIP uptake was different between EV and KD in the other two tumor models. Data are made publicly available on cancerdata.org (http://dx.doi.org/10.17195/candat.2015.10.6). 


\section{Discussion:}

MMP2 is considered as a promising druggable target for therapy due to its important role in various processes of tumor progression like angiogenesis, invasion and metastasis. Therefore, imaging active MMP2 can serve as a surrogate marker for aggressive phenotypes. Imaging MMP2 activity has been previously reported ${ }^{35,36}$. However, the majority of the studies with activatable probes are difficult to translate into the clinic $^{37}$. Antibody-based imaging has the advantage of selective binding; nonetheless clearance from the blood is slower. In this study we have used an antibody fragment, small immuno protein (SIP) for detecting MMP2 with a faster blood clearance, which thus results in higher TBR. aMMP2-SIP imaging was performed in multiple tumor models with varying MMP2 expression and activity. NIRF imaging of aMMP2-SIP showed that 48 h.p.i. was optimal for imaging with a strong specific signal in the tumor and negligible background. Surprisingly we found that aMMP2-SIP uptake did not exclusively depend on MMP2 expression in our models. Despite of clearly low levels of MMP2 expression in the knock-down models of HT1080 and U373 compared to control, the difference with aMMP2-SIP uptake was not evident; supporting the idea that aMMP2-SIP uptake might not be only due to MMP2 expression. In order to explain the discrepancy in aMMP2-SIP uptake we evaluated tumor microenvironmental parameters such as vasculature, perfusion and hypoxia. However, no major differences were observed that could explain the differences in uptake.

The uptake of the antibody was associated with MMP2 activity in the tumors, suggesting specificity of the antibody towards active MMP2. MMP2 activity in U87 MMP2-KD tumors was significantly lower than the control analogous to the aMMP2-SIP uptake. Interestingly, U87 MMP2KD tumors with reduced MMP2 activity also had a slower tumor growth rate compared to control tumors. No difference in MMP2 activity was found in both HT1080 and U373 between MMP2-KD and the control tumors. We further determined if MMP14 and integrin $\alpha \vee \beta 3$ which have been previously associated with MMP2 activity ${ }^{31-34}$, were affected upon silencing MMP2. No differences were observed between control and MMP2KD for both MMP14 and $\alpha \vee \beta 3$ in both HT1080 and U373 tumors. Interestingly, U87 MMP2-KD tumors with low MMP2 activity also had low $\alpha \vee \beta 3$ expression compared to control tumors correlative to the antibody uptake. However, low level of $\alpha v \beta 3$ expression in U87 MMP2KD was unexpected and needs further investigations. It was described that $\alpha v \beta 3$ can directly promote activation, maturation ${ }^{32}$ and localization $^{33}$ of MMP2 and that MMP2 was recruited prior to $\alpha \mathrm{v} \beta 3$ on 
cell surface before migration and MMP2 was necessary for $\alpha v \beta 3$ mediated migration ${ }^{38}$.

Altogether, our study suggests that aMMP2-SIP uptake depends on MMP2 activity. Inhibition of MMP2 appears to be complex due to various mechanisms of activation ${ }^{39}$ and interaction with other molecules such as $\alpha v \beta 3$. However if MMP2 activity is detected specifically, it can be beneficial to monitor tumor progression and tumor responses to treatment. In this study, we have demonstrated that aMMP2-SIP uptake was dependent on MMP2 activity in various in vivo genetic models. Earlier, our lab successfully used Zirconium-89 $\left(\mathrm{Zr}^{89}\right)$ labeled antibodies for positron emission tomography (PET) imaging ${ }^{40}$. In future, to validate aMMP2-SIP as a potential imaging biomarker by using positron emission tomography (PET), labeling the antibody with $\mathrm{Zr}^{89}$ will be performed for feasibility into clinic.

\section{Conclusions:}

The optimal time point for imaging with aMMP2-SIP, an antibody targeting the catalytic domain of MMP2, was $48 \mathrm{hpi}$ with complete background clearance. The uptake of aMMP2-SIP was not solely dependent on MMP2 expression but rather associated with MMP2 activity. Therefore, aMMP2-SIP can be a potential imaging biomarker for detecting MMP2 activity in tumors. 


\section{Materials and methods:}

Cell lines: A549 (ATCC ${ }^{\circledR}$ CCL-185 $^{\mathrm{TM}}$ ), MDA-MB-231(ATCC ${ }^{\circledR} \mathrm{HTB}^{2}$ (6 $^{\mathrm{TM}}$ ), HCT116 (ATCC ${ }^{\circledR}$ CCL-247 $^{\mathrm{TM}}$ ), HT-29 (ATCC $^{\circledR}$ HTB-38 $^{\mathrm{TM}}$ ), Hela $\left(\right.$ ATCC $^{\circledR} \mathrm{CCL}^{-}$ $2^{\text {TM }}$ ), U20S (ATCC ${ }^{\circledR}$ HTB-96 $^{\text {TM}}$ ), U373 (ATCC ${ }^{\circledR}$ HTB-17), U87 (ATCC $^{\circledR} \mathrm{CCL}$

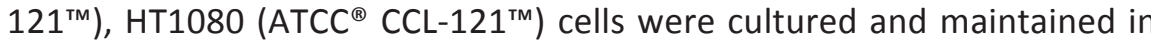
Dulbecco's modified Eagle medium (DMEM) supplemented with $10 \%$ fetal calf serum (FCS). MCF-7 (ATCC ${ }^{\circledR}$ HTB-22 ${ }^{\text {TM}}$ ) cells were cultivated in $10 \%$ FCS containing RPMI 1640 medium and U373 (ATCC ${ }^{\circledR}$ HTB-17) cells in alpha mem supplemented with $10 \%$ FCS and $1 \mu \mathrm{m} / \mathrm{ml}$ L-Glutamine (Westburg).

Tumor models: We engineered stable knock-down (KD) models of MMP2 in HT1080, U373 and U87 by cloning short hairpin RNAs (shRNAs) specific for MMP2 (TRCN0000051526, target sequence GCAGACATCATGATCAACTTT) into the lentiviral vector pLKO.1 (Sigma). Control cells were made by infecting cells with pLKO.1 without insert (EV). $2 \times 10^{6}$ cells diluted in $50 \mu$ of matrigel were injected subcutaneously in the lateral flank of NMRI-nu mice per tumor. Tumor growth was monitored by caliper measurements.

Real-time PCR Analysis: MMP2 mRNA abundance was measured using MMP2 primers with sequence: 5'-CTTCCAAGTCTGGAGCGATGT-3' (forward) and 5'-TACCGTCAAAGGGGTATCCAT-3' (reverse). MMP2 mRNA levels were normalized to the endogenous reference gene $18 \mathrm{~S}$ ribosomal RNA using the primers 5'-AGTCCCTGCCCTTTGTACACA-3' (forward) and 5'GATCCGAGGGCCTCACTAAAC-3' (reverse).

Western blotting and Zymography: Protein was isolated from total cell lysates and loaded with Laemmli buffer in 10\% SDS-PAGE gels and transferred to nitrocellulose membranes (GE healthcare). Proteins were detected using anti-MMP2 (1:1000) (Biomol), MMP14 (1:1000) (Cedarlane) and anti-actin (1:200000) (MP Biomedicals) . Zymography was performed as described before ${ }^{41}$. Cells were incubated overnight in serum-free medium which was collected, concentrated using Amicon ultra centrifugal filter (Ultracel $30 \mathrm{kDa}$ ) and loaded in the zymogram. For ex vivo zymography and western blotting, tumor lysates were prepared by homogenizing tumor samples in radioimmunoprecipitation assay buffer (RIPA buffer) and $20 \mu \mathrm{g}$ of protein was loaded in gels. Gelatin zymogram (10\%), zymogram developing buffer, renaturing buffer and simply blue stain were purchased from Invitrogen. Active MMP2 protein expression and MMP2 activity was quantified by Image J (1.48v, 64 bit) software. 
Transwell invasion assay: $\mathrm{BD}$ Falcon ${ }^{\mathrm{TM}}$ cell culture inserts were coated with $1 \mathrm{mg} / \mathrm{ml}$ of Matrigel diluted in serum-free medium and incubated at $37^{\circ} \mathrm{C}$ overnight. $10^{5}$ cells suspended in serum-free medium were seeded in the inner chamber and $10 \%$ fetal calf serum containing medium was added as chemoattractant in the lower chamber. The invasion system was incubated overnight allowing cell invasion. Cells on the lower side of the insert were stained with $0.5 \%$ crystal violet (in 20\% methanol) and cells were counted manually.

aMMP2-SIP antibody production: aMMP2-SIP was produced against the catalytic domain of MMP2 as described before ${ }^{30}$. Monoclonal cells in suspension were cultured in PowerCHO-2 CD medium (Lonza). Purification of the antibody from the culture medium was done by affinity chromatography using protein A Sepharose Fast Flow resin (GE Healthcare) and a protein gel for purity. ELISA and/or SPR (Biacore 3000) were performed for determining functionality before and after the labelling with Cy5. The concentration and labelling ratio was determined using optical density at different wavelengths. aMMP2-SIP was labeled (average of 2-3 labels per molecule) with Cy5 (lumiprobe) dye according to manufacturer's protocol. The biodistribution of the antibody was described elsewhere ${ }^{29,30}$.

Imaging aMMP2-SIP: Image acquisition and analysis were done as previously described ${ }^{42}$. Near infrared fluorescence (NIRF) imaging was performed using the Optix MX2 (ART, Advanced Research and Technologies) with excitation at $650 \mathrm{~nm}$ and emission at $670 \mathrm{~nm} .75 \mu \mathrm{g}$ of aMMP2-SIP was injected intravenously (i.v.) and imaging was performed at various time points i.e. $0.5,2,4,8,24$ and 48 hour post injection of tracer (h.p.i). A blank scan was performed prior to the injection of tracer to enable autofluorescence correction. Delineation of tumor and background was performed using ART Optix Optiview software. Regions of interest (ROI) were drawn on the lateral side of upper thorax for obtaining background signal distant from the tumors and excluding regions of clearance organs. ROIs drawn on blank scan for both tumor and background were copied to later time points and therefore were kept constant at all time points. Tumor to background ratios (TBR) were calculated at all time points after correction for auto-fluorescence. After imaging, animals were injected with pimonidazole hypoxia marker [60 $\mathrm{mg} / \mathrm{kg}$, intraperitoneal (i.p) Bioconnect] and Hoechst 33342 perfusion marker (15 mg/kg, i.v., Sigma) $1 \mathrm{~h}$ and $1 \mathrm{~min}$ prior to tumor excision, respectively for histological investigations. The animal experiments were approved by animal ethical committee of Maastricht University and were 
in accordance with the institutional guidelines for animal welfare.

Immunohistochemistry: $7 \mu \mathrm{m}$ frozen sections were air dried, acetone fixed and rehydrated by washing with phosphate buffered saline - tween (PBS-Tw-0.2\%). Tumor sections were blocked using 5\% normal goat serum (NGS) (Vector labs) followed by incubation with primary antibodies diluted in PBS-Tw at $4^{\circ} \mathrm{C}$ overnight. After washing with PBS, the sections were incubated with secondary antibodies for $1 \mathrm{~h}$ at room temperature. Primary antibodies that were used are rabbit anti-integrin $\alpha v \beta 3$ antibody (1:250, Abbiotec), mouse anti-MMP2 (4D3) (1:100, Santa Cruz biotechnology), rabbit anti-pimonidazole (1:250, Bio-connect) and rat anti-mouse CD31 (1:500, BD biosciences). Secondary antibodies were, goat anti-rabbit Alexa 488 for $\alpha v \beta 3$ (1:500), goat anti-mouse Alexa 594 for MMP2 (1:500), goat anti-rabbit Alexa 594 for pimonidazole (1:500) and goat anti-rat Alexa 488 for CD31 (1:750) (Invitrogen). Slides were mounted with Shandon ${ }^{\mathrm{TM}}$ Immu-Mount ${ }^{\mathrm{TM}}$. Photomicrographs were acquired using an Olympus BX51WI fluorescence microscope equipped with a Hamamatsu EM-CCD C9100 digital camera, a motorized stage (Ludl Mac 2000) and a 10x objective. Micromanager 1.4 software was used for automated image acquisition ${ }^{43}$. Stitching of images and quantitative analyses were performed using ImageJ version $1.48 \mathrm{v}$ (http://rsb.info.nih.gov/ij/) by an investigator blinded to the subject coding. Total tumor and necrotic areas were manually delineated. MMP2 and $\alpha v \beta 3$ were quantified by visual scoring by two independent investigators (blinded for the treatment conditions) in the total tumor area, where scores from 0 (negative staining) to 5 (high intensity) were used. Percent pimonidazole positive area (hypoxic fraction), relative vascular area and proportion of the perfused vessels were determined in viable tumor compartment as described previously ${ }^{44,45}$.

In situ zymography: Tumor sections were air dried for 30 minutes. Dye quenched gelatin (DQ gelatin) (Invitrogen) was prepared in $1 \% \mathrm{w} / \mathrm{v}$ of low melting agarose solution (Sigma) in 1:10 mixture $(100 \mu \mathrm{g} / \mathrm{ml})$ and maintained at $37^{\circ} \mathrm{C} .40 \mu \mathrm{l}$ of this solution was placed directly on top of the section and mounted with a cover slip. The sections were incubated at $37^{\circ} \mathrm{C}$ overnight in a humid environment. The sections were then scanned for MMP2 activity and images were stitched as described above.

Statistics: A nonparametric Mann Whitney test was performed to determine statistical differences in $\alpha v \beta 3$ and MMP2 scores between groups. One sample t-test was used to test whether average change in MMP2 activity is significantly different from 1 . For all other statistical analyses between two groups the unpaired t test was performed. All 
statistics and graphs were made in Graphpad Prism (v5.03). A two- sided $\mathrm{P}$-value smaller than 0.05 was considered statistically significant. 


\section{Acknowledgments}

The micrographs in this paper were taken with a confocal spinning disk microscope financed by The Netherlands Organisation for Scientific Research (NWO), grant number 911-06-003. The authors acknowledge the support of the QuIC-ConCePT project, partly funded by EFPIA companies and the Innovative Medicine Initiative Joint Undertaking (IMI JU) under Grant Agreement No. 115151. We also want to thank Dario Neri (ETH Zürich) for his scientific support.

\section{Competing financial interests:}

The author(s) declare no competing financial interests.

\section{Author contributions statements:}

Kranthi Marella Panth performed the study, analyses and wrote the main manuscript text. Rianne Biemans, Natasja G Lieuwes assisted with experiments. Twan van den Beucken, Ala Yaromina, Mario Losen, Ludwig Dubois and Philippe Lambin provided expert guidance for experiments and data analyses. All authors reviewed the manuscript. 


\section{Figure legends}

Figure 1: Validation of MMP2-KD models in vitro:

(A) Relative MMP2 mRNA expression and (B) MMP2 gelatinase activity in selected cell lines. Recombinant human MMP2 (rhMMP2 66kDa) is taken as positive control and serum free medium as negative control (C) Relative MMP2 mRNA expression, (D) protein expression and (E) active MMP2 protein quantification (F) MMP2 gelatinase activity and (G) quantification of MMP2 gelatinase activity of empty vector control (EV) and MMP2 knockdown (KD) cells of HT1080, U373 and U87 MMP2-KD compared to control cells. $(H)$ Percentage of invading cells in invasive cell lines HT1080 and U373. Data represents the mean +/- SD of at least 3 independent experiments. ( $* * * P<0.0001, * * P<0.01, * P<0.05$ ) 
A

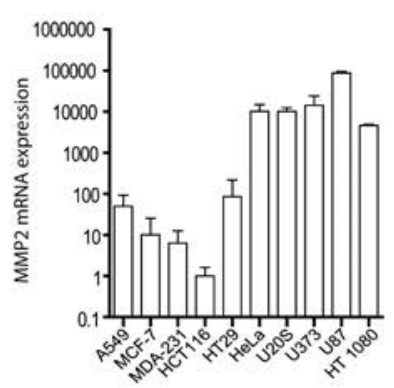

D

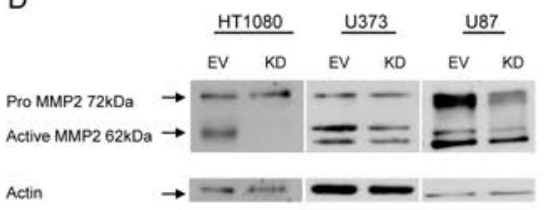

E

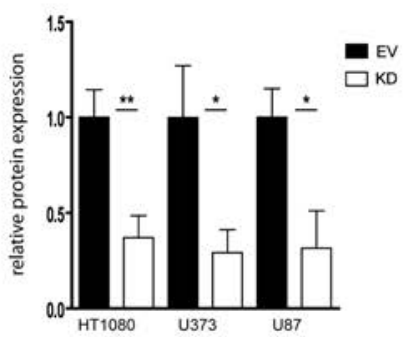

B

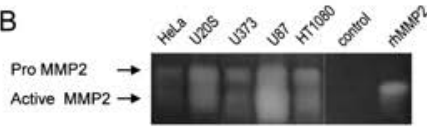

C

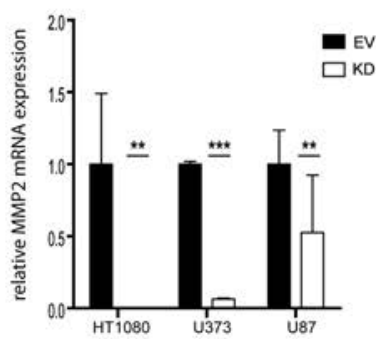

F

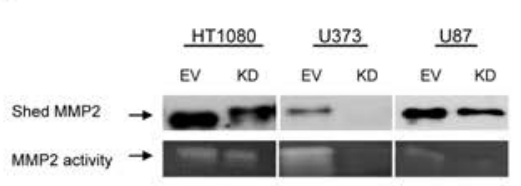

G

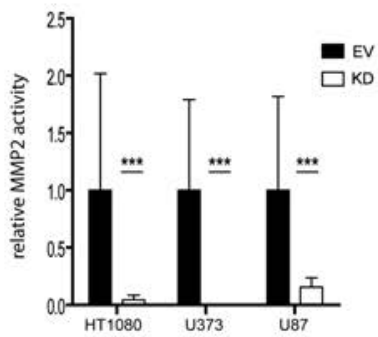

H

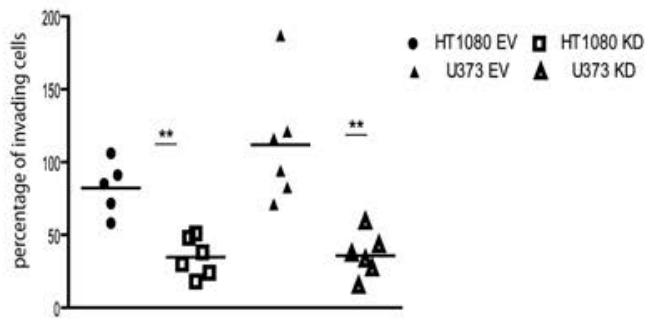


Figure 2: aMMP2-SIP uptake over time in selected models

(A) Representative blank (pre-injection) corrected images of HCT116 ( $n=5)$, HT1080 ( $n=13)$, U373 $(n=6)$ and U87 $(n=8)$ tumor-bearing mice at $0.5,2,4,8,24$ and 48 hours post injection of tracer. Black arrow indicates tumor, red arrow indicates background. (B) Tumor to background ratio quantification over time in different models. $(* \mathrm{P}<0.05)$

A
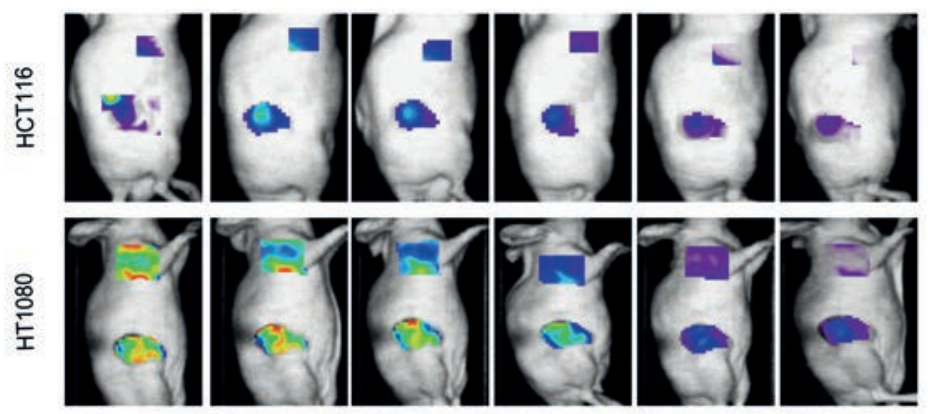

$\operatorname{Max}$
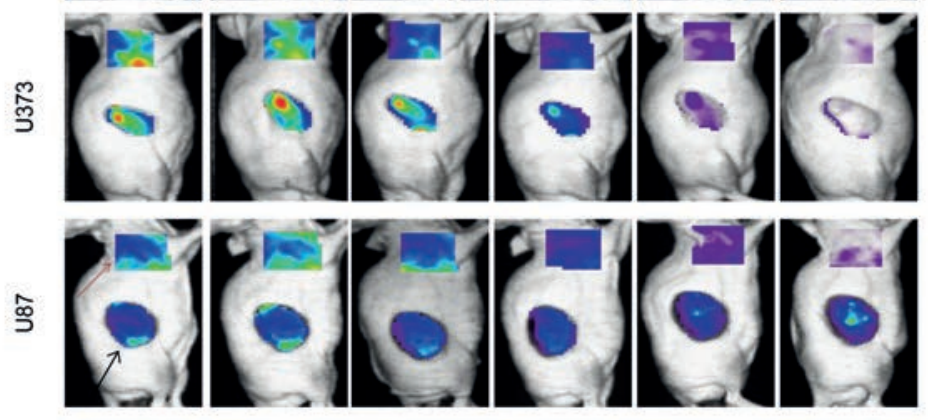

Min

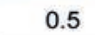

2

4

8

24

48

Time (hours)

B

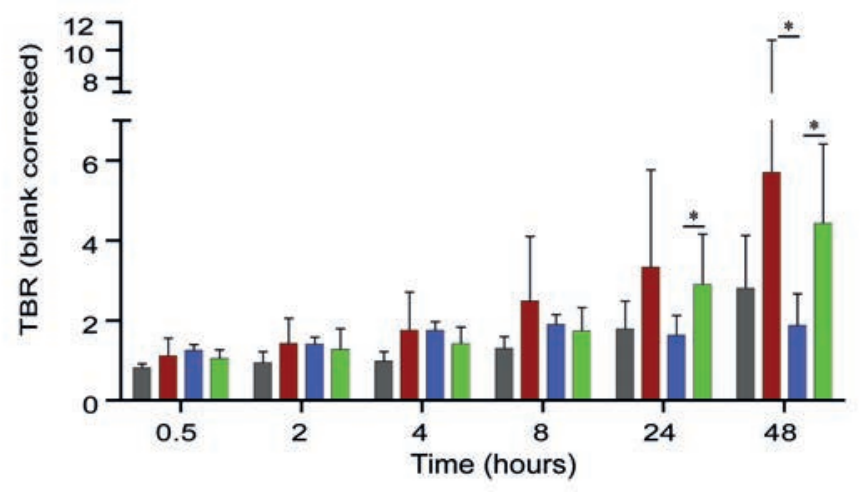


Figure 3: aMMP2-SIP uptake in MMP2 knock-down models compared to control

Representative images at optimal time point 48h.p.i. (Black arrow indicates tumor, red arrow indicates background) and TBR at various time points for (A) HT1080 (control $n=13$, MMP2-KD n=16), (B) U373 (control $n=6, M M P 2-K D ~ n=6$ ) and (C) U87 (control $n=8$, MMP2-KD $\mathrm{n}=15)\left({ }^{* * *} \mathrm{P}<0.001, * * \mathrm{P}<0.01\right)$.

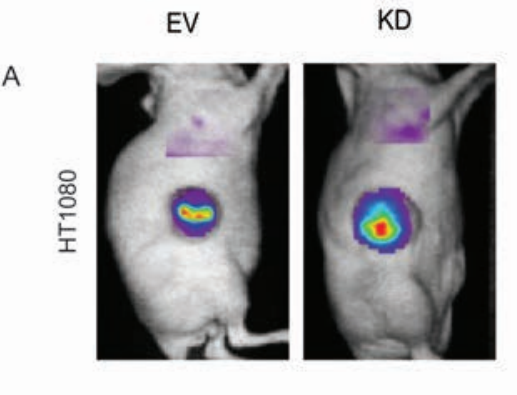

Max

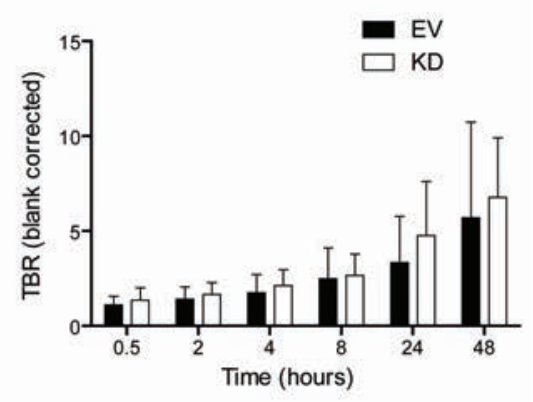

B
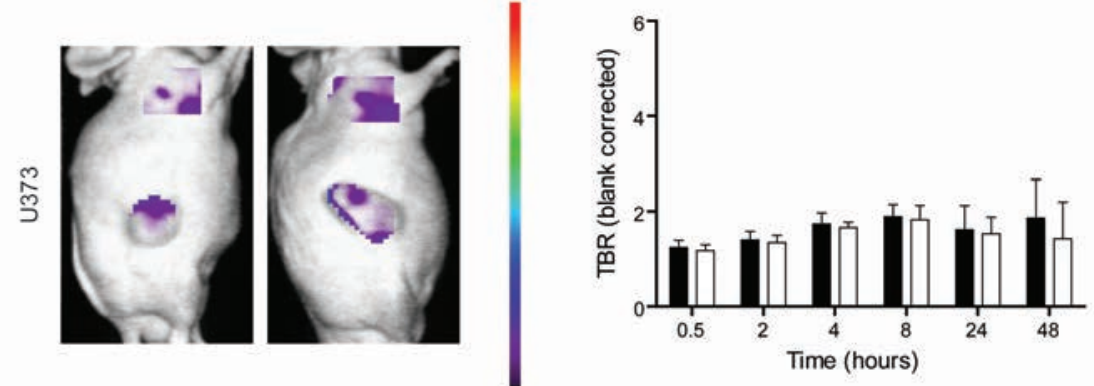

C
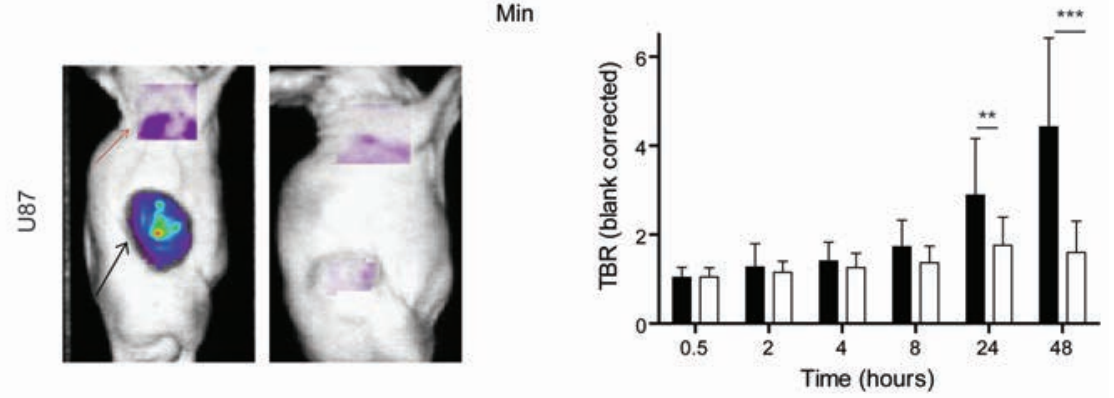
Figure 4: MMP2 activity in tumors

(A) MMP2 activity in tumor lysates determined by gelatin zymography. Data represent the mean +/- SD of atleast 5 samples. (B) Representative images (green: $\alpha v \beta 3$, red: MMP2) and corresponding $\alpha \mathrm{v} \beta 3$ score for MMP2 knock-down (KD) tumors compared to control (EV) for HT1080, U373 tumors and U87 ( $\left.{ }^{*} P<0.05\right)$. Data represents median of at least 5 samples (median with 5 and 95 percentile). (C) Representative images of in situ gelatin zymography on U87 (control) tumor section showing MMP2 activity (Top). Bottom images show regions of high MMP2 activity corresponding with overlapping regions of $\alpha v \beta 3$ (green) and MMP2 (red).

A

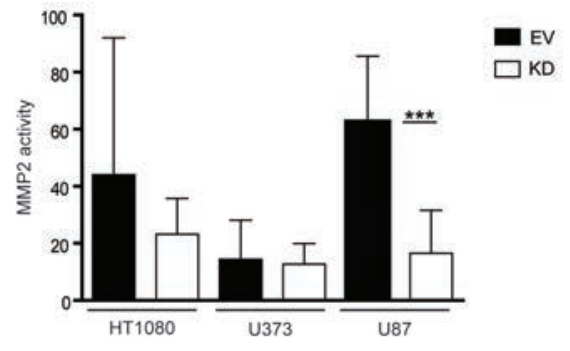

B
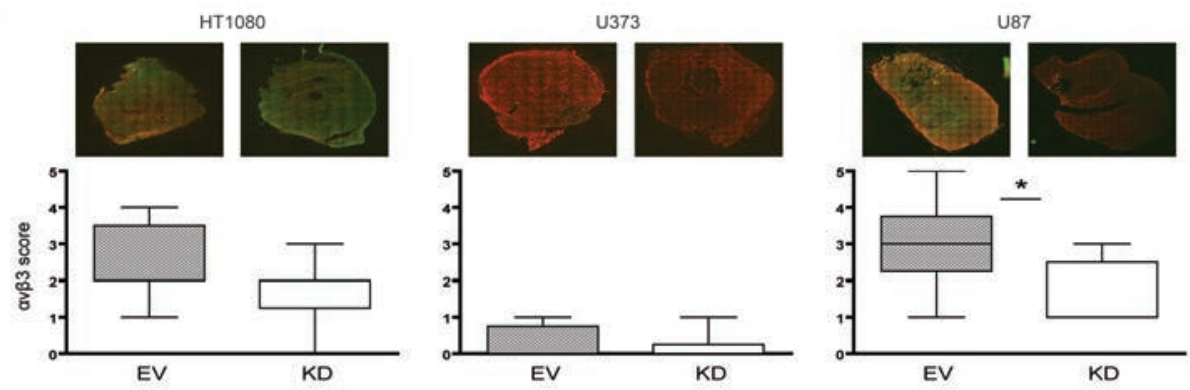

C

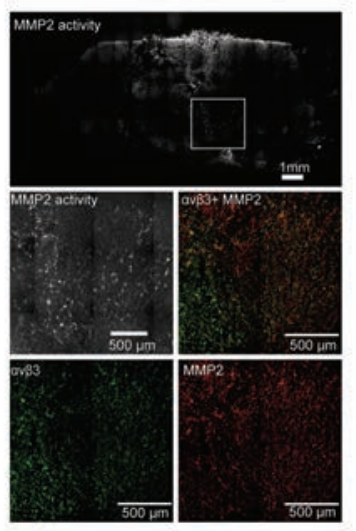


Figure 5: Tumor growth

Tumor doubling time of knock-down (KD) tumors compared to control (EV) for HT1080, U87 and U373. Data represents mean +/- SD $(* * P<0.01)$.

HT1080

U373

U87
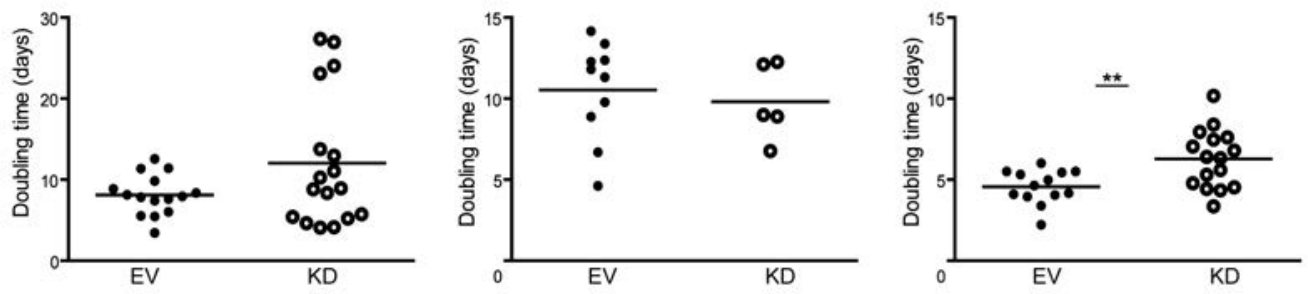


\section{Supplementary information:}

\section{Supplementary figure 1: MMP2 expression in tumors}

MMP2 mRNA expression determined by qPCR (mean +/- SD) and MMP2 score (median with 5 and 95 percentile of atleast 5 samples) determined by immunohistochemistry in HT1080, U373 and U87 control (EV) and MMP2-KD (KD) tumors ( $* * * P<0.0001, * * P<0.01, * P<0.05)$.

\section{HT1080}
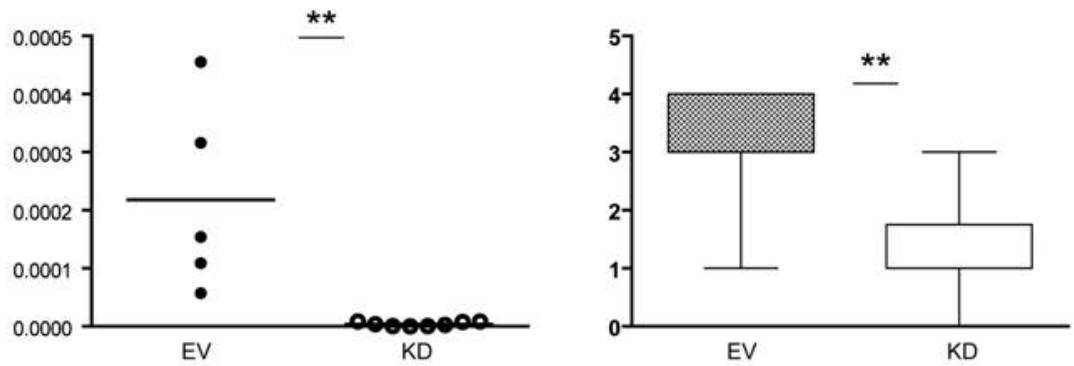

U373
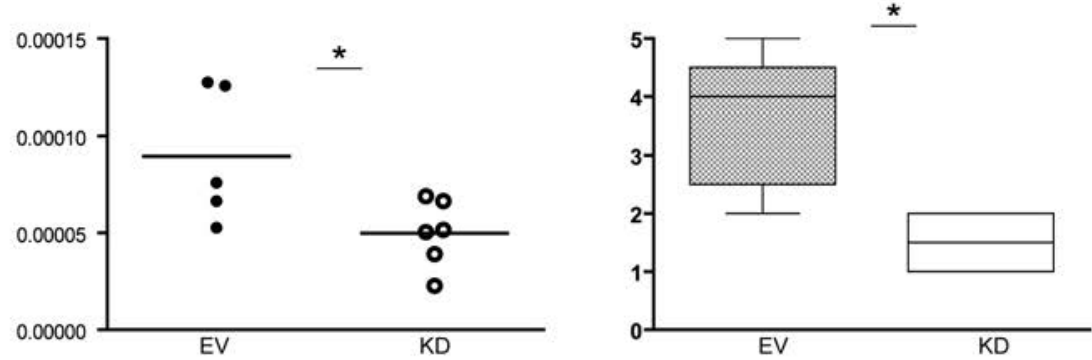

U87
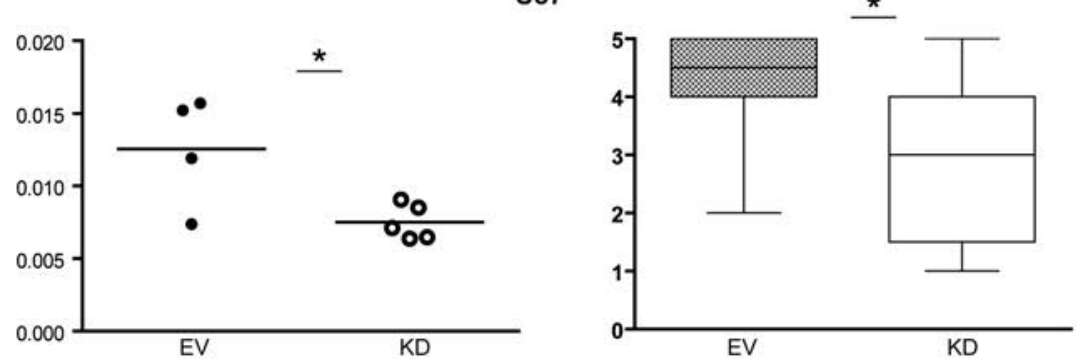
Supplementary figure 2: Representative images for tumor microenvironment

Immunohistochemistry images of HT1080, U373 and U87 control (EV) and MMP2-KD (KD) tumors stained for Hypoxia [pimonidazole (pimo)] in green, blood vessels (CD31) in red and perfused vessels (Hoechst) in blue. White bar represents a scale of $1 \mathrm{~mm}$.

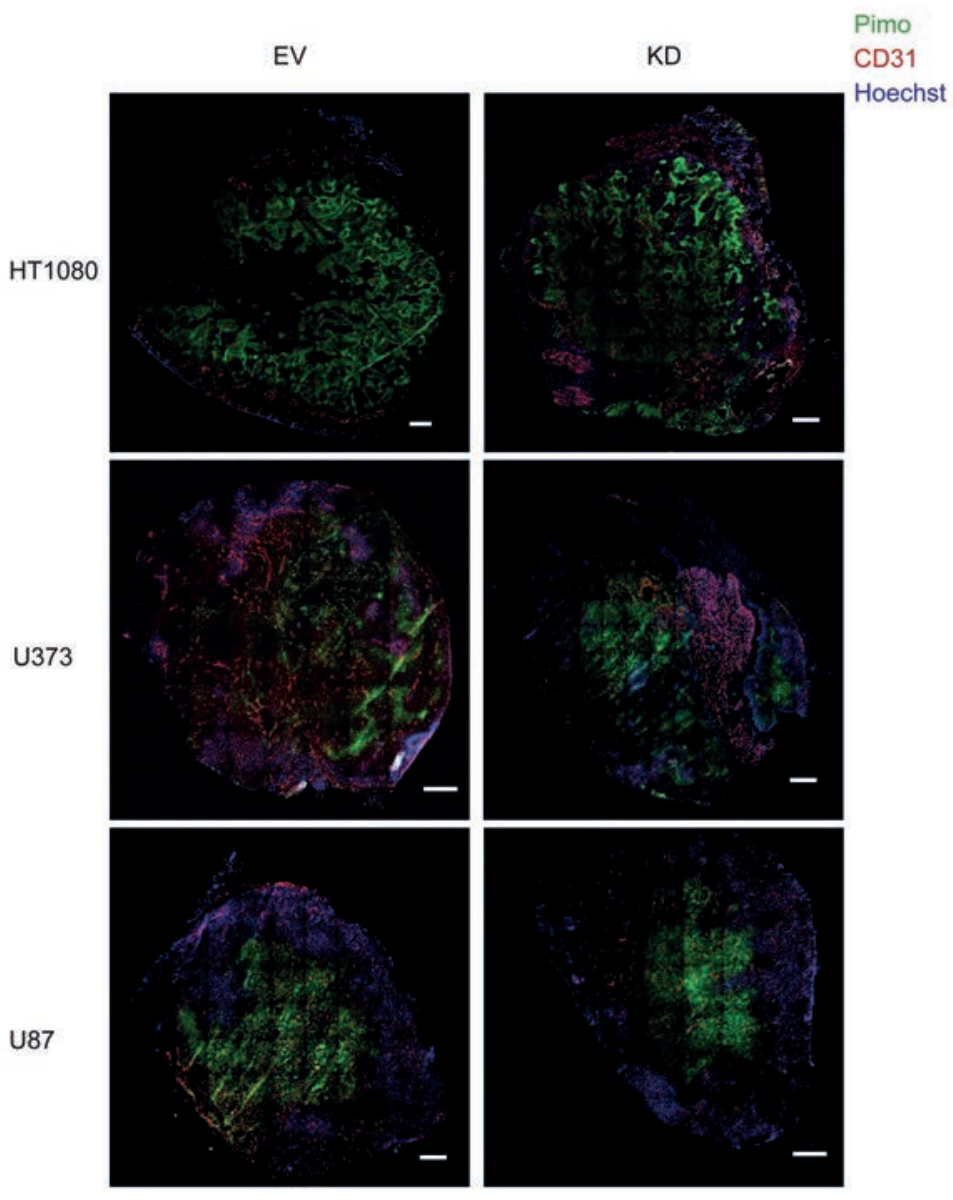


(A) Quantification of the hypoxic fraction, microvessel density and the percent perfused vessels in HT1080 U373 and U87 control (EV) and MMP2-KD (KD) tumors. (B) Western blot analysis and (C) quantification of active MMP14 expression in tumor lysates. Data represents mean +/- SD of at least 5 samples.

A
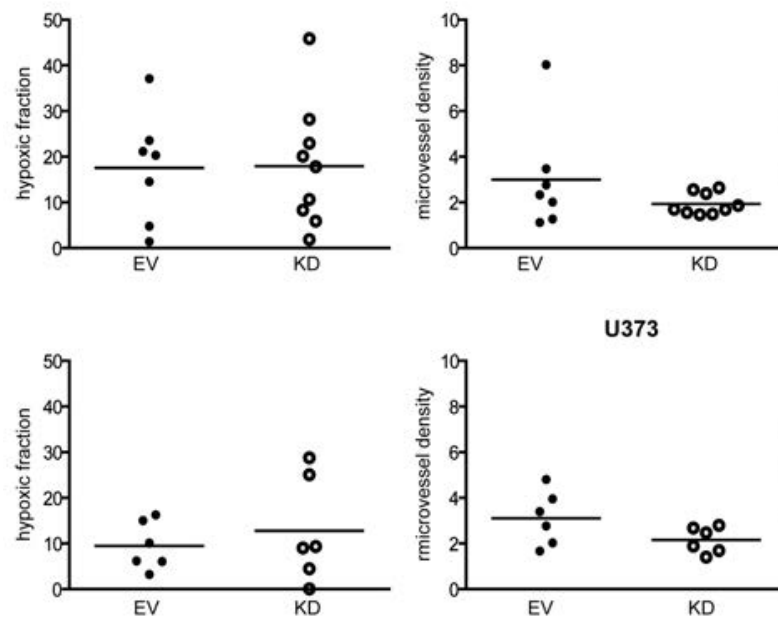

U373

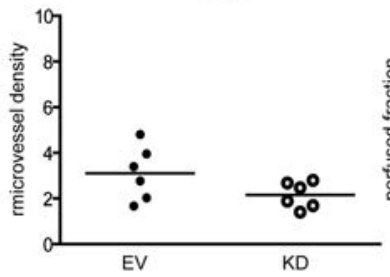

U87
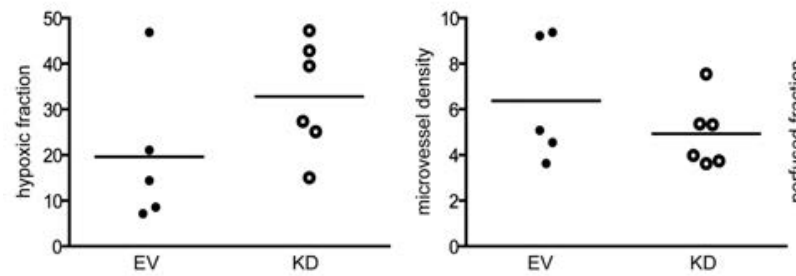

B

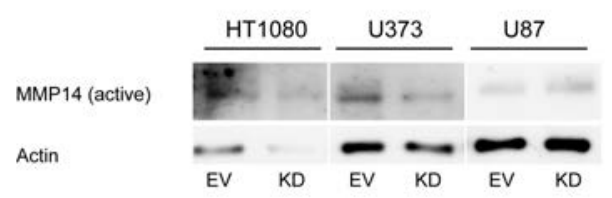

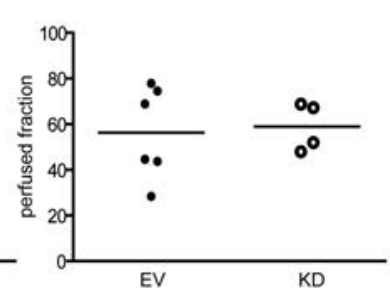
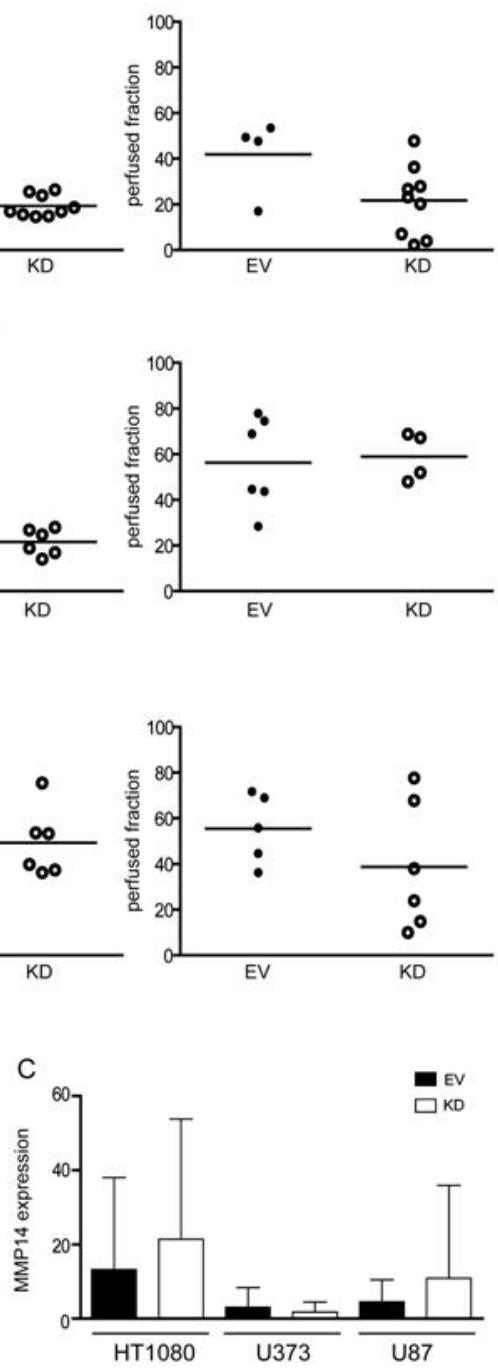


\section{References:}

1 Löffek, S., Schilling, O. \& Franzke, C.-W. Biological role of matrix metalloproteinases: a critical balance. European Respiratory Journal 38, 191-208, doi:10.1183/09031936.00146510 (2011).

2 Johansson, N., Ahonen, M. \& Kähäri *, V. M. Matrix metalloproteinases in tumor invasion. Cellular and Molecular Life Sciences CMLS 57, 5-15, doi:10.1007/s000180050495 (2000).

3 Kessenbrock, K., Plaks, V. \& Werb, Z. Matrix Metalloproteinases: Regulators of the Tumor Microenvironment. Cell 141, 52-67 (2010).

4 Zucker, S. \& Cao, J. Selective matrix metalloproteinase (MMP) inhibitors in cancer therapy: Ready for prime time? Cancer biology \& therapy 8, 2371-2373 (2009).

5 Decock, J., Thirkettle, S., Wagstaff, L. \& Edwards, D. R. Matrix metalloproteinases: protective roles in cancer. Journal of Cellular and Molecular Medicine 15, 12541265, doi:10.1111/j.1582-4934.2011.01302.x (2011).

6 McCawley, L. J. \& Matrisian, L. M. Matrix metalloproteinases: they're not just for matrix anymore! Current Opinion in Cell Biology 13, 534-540 (2001).

7 Roomi, M. W., Monterrey, J. C., Kalinovsky, T., Rath, M. \& Niedzwiecki, A. Patterns of MMP-2 and MMP-9 expression in human cancer cell lines. Oncology Reports 21, 1323-1333, doi:10.3892/or_00000358 (2009).

8 Wang, M., Wang, T., Liu, S., Yoshida, D. \& Teramoto, A. The expression of matrix metalloproteinase-2 and-9 in human gliomas of different pathological grades. Brain Tumor Pathology 20, 65-72, doi:10.1007/bf02483449 (2003).

9 Hofmann, U. B. et al. Matrix metalloproteinases in human melanoma cell lines and xenografts: increased expression of activated matrix metalloproteinase-2 (MMP-2) correlates with melanoma progression. Br J Cancer 81, 774-782, doi:10.1038/sj.bjc.6690763 (1999).

10 Väisänen, A. et al. Matrix metalloproteinase-2 (MMP-2) immunoreactive protein-a new prognostic marker in uveal melanoma? The Journal of Pathology 188, 56-62, doi:10.1002/(sici)1096-9896(199905)188:1<56::aidpath304>3.0.co;2-b (1999).

11 Pellikainen, J. M. et al. Expression of Matrix Metalloproteinase (MMP)-2 and MMP-9 in Breast Cancer with a Special Reference to Activator Protein-2, HER2, and Prognosis. Clinical Cancer Research 10, 7621-7628, doi:10.1158/10780432.ccr-04-1061 (2004).

12 Emmert-Buck, M. R. et al. Increased gelatinase A (MMP-2) and cathepsin B activity in invasive tumor regions of human colon cancer samples. The American journal of pathology 145, 1285-1290 (1994).

13 Fang, J. et al. Matrix metalloproteinase-2 is required for the switch to the angiogenic phenotype in a tumor model. Proceedings of the National Academy of Sciences of the United States of America 97, 3884-3889 (2000).

14 ZHENG, H. et al. Expressions of MMP-2, MMP-9 and VEGF are Closely Linked to Growth, Invasion, Metastasis and Angiogenesis of Gastric Carcinoma. Anticancer Research 26, 3579-3583 (2006).

15 Munoz-Najar, U. M., Neurath, K. M., Vumbaca, F. \& Claffey, K. P. Hypoxia stimulates breast carcinoma cell invasion through MT1-MMP and MMP-2 activation. Oncogene 25, 2379-2392 (2005).

16 Schmalfeldt, B. et al. Increased Expression of Matrix Metalloproteinases (MMP)2, MMP-9, and the Urokinase-Type Plasminogen Activator Is Associated with Progression from Benign to Advanced Ovarian Cancer. Clinical Cancer Research 7, 2396-2404 (2001). 
17 Koshiba, T. et al. Involvement of matrix metalloproteinase-2 activity in invasion and metastasis of pancreatic carcinoma. Cancer 82, 642-650, doi:10.1002/(sici)1097-0142(19980215)82:4<642::aid-cncr5>3.0.co;2-n (1998).

18 Badiga, A. V. et al. MMP-2 siRNA Inhibits Radiation-Enhanced Invasiveness in Glioma Cells. PLoS ONE 6, e20614, doi:10.1371/journal.pone.0020614 (2011).

19 Chetty, C., Bhoopathi, P., Rao, J. S. \& Lakka, S. S. Inhibition of matrix metalloproteinase-2 enhances radiosensitivity by abrogating radiation-induced FoxM1-mediated G2/M arrest in A549 lung cancer cells. International Journal of Cancer 124, 2468-2477, doi:10.1002/ijc.24209 (2009).

20 Kargiotis, O. et al. Adenovirus-mediated transfer of siRNA against MMP-2 mRNA results in impaired invasion and tumor-induced angiogenesis, induces apoptosis in vitro and inhibits tumor growth in vivo in glioblastoma. Oncogene 27, 48304840 (2008).

21 Prontera, C., Mariani, B., Rossi, C., Poggi, A. \& Rotilio, D. Inhibition of gelatinase A (MMP-2) by batimastat and captopril reduces tumor growth and lung metastases in mice bearing Lewis lung carcinoma. International Journal of Cancer 81, 761766, doi:10.1002/(sici)1097-0215(19990531)81:5<761::aid-ijc16>3.0.co;2-1 (1999).

22 Wu, A. M. \& Olafsen, T. Antibodies for Molecular Imaging of Cancer. The Cancer Journal 14, 191-197, doi:10.1097/PPO.0b013e31817b07ae (2008).

23 van Dongen, G. A. M. S., Visser, G. W. M., Lub-de Hooge, M. N., de Vries, E. G. \& Perk, L. R. Immuno-PET: A Navigator in Monoclonal Antibody Development and Applications. The Oncologist 12, 1379-1389, doi:10.1634/theoncologist.12-121379 (2007).

24 Olafsen, T. \& Wu, A. M. Antibody Vectors for Imaging. Seminars in Nuclear Medicine 40, 167-181 (2010).

25 Holliger, P. \& Hudson, P. J. Engineered antibody fragments and the rise of single domains. Nat Biotech 23, 1126-1136 (2005).

26 Kaur, S. et al. Recent trends in antibody-based oncologic imaging. Cancer Letters 315, 97-111 (2012).

27 Dana Jones, S. \& Marasco, W. A. Antibodies for targeted gene therapy: extracellular gene targeting and intracellular expression. Advanced Drug Delivery Reviews 31, 153-170 (1998).

28 Borsi, L. et al. Selective targeting of tumoral vasculature: Comparison of different formats of an antibody (L19) to the ED-B domain of fibronectin. International Journal of Cancer 102, 75-85, doi:10.1002/ijc.10662 (2002).

29 Pfaffen, S., Frey, K., Stutz, I., Roesli, C. \& Neri, D. Tumour-targeting properties of antibodies specific to MMP-1A, MMP-2 and MMP-3. European Journal of Nuclear Medicine and Molecular Imaging 37, 1559-1565, doi:10.1007/s00259-010-1446-9 (2010).

30 Pfaffen, S., Hemmerle, T., Weber, M. \& Neri, D. Isolation and characterization of human monoclonal antibodies specific to MMP-1A, MMP-2 and MMP-3. Experimental Cell Research 316, 836-847 (2010).

31 Nishida, Y. et al. Activation of Matrix Metalloproteinase-2 (MMP-2) by Membrane Type 1 Matrix Metalloproteinase through an Artificial Receptor for ProMMP-2 Generates Active MMP-2. Cancer Research 68, 9096-9104, doi:10.1158/0008-5472.can-08-2522 (2008).

32 Deryugina, E. I. et al. MT1-MMP Initiates Activation of pro-MMP-2 and Integrin $\alpha v \beta 3$ Promotes Maturation of MMP-2 in Breast Carcinoma Cells. Experimental Cell Research 263, 209-223 (2001).

33 Brooks, P. C. et al. Localization of Matrix Metalloproteinase MMP-2 to the Surface of Invasive Cells by Interaction with Integrin av $\beta 3$. Cell 85, 683-693 (1996). 
34 Hofmann, U. B., Westphal, J. R., Van Kraats, A. A., Ruiter, D. J. \& Van Muijen, G. N. P. Expression of integrin $\alpha v \beta 3$ correlates with activation of membrane-type matrix metalloproteinase-1 (MT1-MMP) and matrix metalloproteinase-2 (MMP2 ) in human melanoma cells in vitro and in vivo. International Journal of Cancer 87, 12-19, doi:10.1002/1097-0215(20000701)87:1<12::aid-ijc3>3.0.co;2-a (2000).

35 van Duijnhoven, S. M. J., Robillard, M. S., Nicolay, K. \& Grüll, H. Tumor Targeting of MMP-2/9 Activatable Cell-Penetrating Imaging Probes Is Caused by TumorIndependent Activation. Journal of Nuclear Medicine 52, 279-286, doi:10.2967/jnumed.110.082503 (2011).

36 Bremer, C., Bredow, S., Mahmood, U., Weissleder, R. \& Tung, C.-H. Optical Imaging of Matrix Metalloproteinase-2 Activity in Tumors: Feasibility Study in a Mouse Model. Radiology 221, 523-529, doi:10.1148/radiol.2212010368 (2001).

37 Lebel, R. \& Lepage, M. A comprehensive review on controls in molecular imaging: lessons from MMP-2 imaging. Contrast Media \& Molecular Imaging 9, 187-210, doi:10.1002/cmmi.1555 (2014).

38 Jiao, Y. et al. Matrix metalloproteinase-2 Promotes $\alpha v \beta 3$ Integrin-Mediated Adhesion and Migration of Human Melanoma Cells by Cleaving Fibronectin. PLoS ONE 7, e41591, doi:10.1371/journal.pone.0041591 (2012).

39 Stetler-Stevenson, W. G. Matrix metalloproteinases in angiogenesis: a moving target for therapeutic intervention. The Journal of Clinical Investigation 103, 1237-1241, doi:10.1172/jci6870 (1999).

40 Aerts, H. J. W. L. et al. Disparity Between In Vivo EGFR Expression and 89ZrLabeled Cetuximab Uptake Assessed with PET. Journal of Nuclear Medicine 50, 123-131, doi:10.2967/jnumed.108.054312 (2009).

$41 \mathrm{Hu}, \mathrm{X}$. \& Beeton, C. Detection of Functional Matrix Metalloproteinases by Zymography. e2445, doi:doi:10.3791/2445 (2010).

42 Dubois, L. et al. Imaging of CA IX with fluorescent labelled sulfonamides distinguishes hypoxic and (re)-oxygenated cells in a xenograft tumour model. Radiotherapy and Oncology 92, 423-428 (2009).

43 Stuurman, N., Edelstein, A. D., Amodaj, N., Hoover, K. H. \& Vale, R. D. Computer Control of Microscopes using $\mu$ Manager. Current protocols in molecular biology / edited by Frederick M. Ausubel ... [et al.] CHAPTER, Unit14.20-Unit14.20, doi:10.1002/0471142727.mb1420s92 (2010).

44 Yaromina, A. et al. Exploratory Study of the Prognostic Value of Microenvironmental Parameters During Fractionated Irradiation in Human Squamous Cell Carcinoma Xenografts. International Journal of Radiation Oncology*Biology*Physics 80, 1205-1213 (2011).

45 Peeters, S. G. J. A. et al. TH-302 in Combination with Radiotherapy Enhances the Therapeutic Outcome and Is Associated with Pretreatment [18F]HX4 Hypoxia PET Imaging. Clinical Cancer Research 21, 2984-2992, doi:10.1158/1078-0432.ccr-150018 (2015). 


\section{Chapter 5}

\section{Radiogenomics: establishing causality between genetics and radiomics}

Published in: Radiother Oncol. 2015 Sep;116(3):462-6

Panth KM*, Leijenaar RT*, Carvalho S, Lieuwes NG, Yaromina A, Dubois L, Lambin $\mathrm{P}$

* Equal contribution 


\section{Abstract:}

Background and Purpose: The central hypothesis of "radiomics" is that imaging features reflect tumor phenotype and genotype. Until now only correlative studies have been performed. The main objective of our study is to determine whether a causal relationship exists between genetic changes and image features. The secondary objective is to assess whether the combination with radiotherapy (RT) influences these image features.

Material and methods: HCT116 doxycycline (dox) inducible GADD34 cells were grown as xenografts in the flanks of NMRI-nu mice. GADD34 overexpression decreases hypoxic fraction. Radiomics analyses were performed on computed tomography images obtained at $40 \mathrm{kVp}$ and again at $80 \mathrm{kVp}$ for validation, before radiotherapy at a volume of $200 \mathrm{~mm}^{3}, 4$ days post RT (10 Gy) and $500 \mathrm{~mm}^{3}$. To select reproducible features test-retest experiments were performed at baseline.

Results: Gene induction and/or irradiation translated into significant changes in radiomics features. Post irradiation, 17 features for $40 \mathrm{kVp}$ and 9 features for $80 \mathrm{kVp}$ differed significantly between dox+ and doxcombined with RT. 8 and 4 of these features remained consistent for 40 and $80 \mathrm{kVp}$, respectively.

Conclusions: Radiomics is able to identify early effects of changed gene expression combined with radiation treatment in tumors with similar volumes which are not visible to human eye. 


\section{Introduction:}

Genomics and proteomics are widely used in the concept of personalized medicine [1,2]. However, tumor heterogeneity, which cannot be determined by invasive biopsies, varies spatially and temporally $[3,4]$. Advancements in medical imaging have provided better diagnostic biomarkers and novel imaging methods that can aid in early diagnosis, patient stratification and the monitoring of treatment responses [5]. Furthermore, three-dimensional tumor heterogeneity is defined by the underlying tumor genotype and phenotype. Therefore, the central hypothesis of radiomics is that features extracted from noninvasive images reflect the underlying phenotype and genotype [6]. Radiomics uses mathematical algorithms to extract a large number of quantitative features from medical images, describing tumor image intensity, shape and texture, capturing intra-tumoral heterogeneity [7] (http://www.radiomics.org; animation: https://youtu.be/VfOF7q8vaS4).

It has previously been shown that radiomics can provide valuable information based on CT images $[6,8]$ that have prognostic value in lung and head and neck cancer [7,9]. Segal has shown an association between defined imaging traits and gene expression profiles of human hepatocellular carcinomas (HCC) [10]. It was also reported that this radiogenomics approach can identify drug-responsive gene expression patterns in HCC [11]. In the context of radiomics, studies so far have shown only a correlation of the radiomics signatures to genetic signatures, but no causal relationship has been established.

The main goal of this study is to provide a proof-of-concept that genetic changes with phenotypic consequences influence image-derived radiomics features. More specifically, we prospectively evaluated whether radiomics features are causally related to genetic factors and whether radiation therapy affects those image features. In addition, we investigated whether the features obtained for all categories are reproducible in test-retest experiments and whether the features found remain consistent across the different acquisition time points. Furthermore, we evaluated the effect of CT energy on radiomics features. 


\section{Materials and Methods:}

Tumor cells and chemicals: Cells were cultured and maintained according to instructions and guidelines from the American Type Culture Collection. Doxycycline hyclate (dox) was purchased from Sigma Aldrich. Construction of HCT116 colorectal carcinoma cells overexpressing GADD34c and control pCDNA5(+) has been described previously [12].

Preclinical imaging studies: All animal experiments were approved by the Animal Ethical Committee of Maastricht University (2013-038). HCT116 tumor cells were subcutaneously injected in matrigel in the lateral flanks of NMRI-nu/nu mice. Mice were randomized after cell injection between doxycycline (dox+, $\mathrm{n}=17)(2 \mathrm{~g} / \mathrm{L}$ in $5 \%$ sucrose in drinking water) and placebo (dox-, $n=16$ ) administration to induce expression of the gene of interest. HCT116 pCDNA5(+) xenografts were included to assess features related to dox administration without gene induction. CT images were obtained at two different energies ( 40 and $80 \mathrm{kVp}$ ) using the Precision $\mathrm{X}$ ray $X-R A D 225 C x$ at pre (baseline), at 4 days post radiation therapy (RT) and at a tumor volume of $500 \mathrm{~mm}^{3}$. The number of animals allocated per group after baseline scans were: dox+RT- $(n=8)$, dox+RT+ $(n=9)$, doxRT- $(n=7)$, dox- RT+ $(n=9)$. Images were reconstructed with an isotropic voxel size of $0.1 \mathrm{~mm}$. Tumors were irradiated with a single dose of $10 \mathrm{~Gy}$ at an average tumor volume of $210(+/-36) \mathrm{mm}^{3}$. A second scan (retest) was acquired 5 minutes after the first (test) scan at baseline by repositioning the mouse in the scanner to determine feature test-retest (TRT) variability (Figure 1).

Tumor delineation and image analysis: Tumors were delineated using SmART-Plan treatment planning software [13] . In order to acquire interobserver variability (IOV), two experts independently delineated tumors from baseline images. For each image, we determined 625 radiomics features, grouped into five groups: 1) tumor intensity, 2) shape, 3) texture, 4) wavelet features, and 5) Laplacian of Gaussian (LoG) features. Features from group 1 describe the first-order histogram of all voxel intensity values in the tumor volume. Group 2 features describe the three-dimensional shape and size of a tumor. Features from group 3 quantify texture within the tumor image calculated from gray-level cooccurrence (GLCM), gray-level run-length (GLRLM), and gray-level sizezone texture matrices (GLSZM). Group 4 consists of features from group 1 and 2, after a wavelet decomposition of the image. Group 5 consists of features from group 1, calculated after applying Laplacian of Gaussian filtering to the image, highlighting image regions at different scales. Texture matrices were determined by considering 26 connected voxels 
(i.e. voxels were considered to be neighbors in all 13 directions in three dimensions) at a distance of 1 voxel. The features derived from GLCM and GLRLM were calculated by averaging their value over all 13 directions. LoG features were determined at a filter standard deviation ranging from 0.1-0.5 $\mathrm{mm}$ with $0.05 \mathrm{~mm}$ increments. Image analysis was performed in Matlab R2012b (The Mathworks, Natick, MA) using an adapted version of CERR [14] and Radiomics software developed in-house to extract imaging features. A detailed description and mathematical definitions of the radiomics features assessed in this study are described elsewhere $[9,15]$.

Feature selection: The intra-class correlation coefficient (ICC) [16] was calculated to provide an indication of both the test-retest (TRT) and interobserver (IOV) reliability of feature measurements. The ICC is a statistical measure between 0 and 1, where 0 indicates no reliability and 1 indicates perfect reliability. For the ICC regarding the TRT reliability of imaging features, we used the definition of ICC $(1,1)$. The ICC for IOV was determined by the definition of $\operatorname{ICC}(2,1)$. To determine the ICCS, we obtained variance estimates by partitioning the total variance by means of analysis of variance (ANOVA). Absolute variability was furthermore estimated as the smallest detectible change (SDC) [17]. To provide a basis for evaluating the magnitude of the TRT and IOV SDC values, we determined a coefficient of reliability (COR) by normalizing SDC to a percentage of the range of feature values ( $2.5-97.5$ percentile) over all lesions included. For each feature, we determined the average TRT and IOV ICC rank and selected the resulting 50\% top-ranked features for further analysis. We performed feature selection using R (version 3.1.0).

Statistical analyses: We performed a Wilcoxon rank sum test to compare feature values between two groups using $R$ (version 3.1.0). We used a non-parametric T-test in Graphpad Prism v 5.03 to perform a statistical analysis to compare the tumor growth rates between the two groups. A P-value $<0.05$ was considered significant. 


\section{Results:}

Upon GADD34 gene induction $(\mathrm{dox}+)$, no difference $(P=0.405)$ in tumor growth was observed compared to animals treated with placebo (dox-). However, upon irradiation (single dose 10Gy), the time to reach 4 times start volume for these doxycycline treated tumors was significantly $(\mathrm{P}<0.05)$ increased upon irradiation (single dose 10Gy) compared to doxirradiated tumors (Figure 2).

Image-derived quantitative features need to be robust and reproducible in order to provide reliable measurements. We therefore selected the $50 \%$ top-ranked features based on their average ICC for the TRT variability (ICC1) and inter-observer variability (ICC 2) (Figure 3). In the control pCDNA5(+) group, 12 features were found to differ significantly $(\mathrm{P}<0.05)$ between dox+ and dox- treatment groups. This suggests that these features were related to doxycycline administration. They were therefore eliminated in further analyses, which left 303 remaining features.

We first evaluated features from $40 \mathrm{kVp}$ CT images for validating our hypothesis. We determined features that were significantly different between dox+ and dox- GADD34 tumors to investigate which image features are influenced by genetic factors. At a tumor volume of $200 \mathrm{~mm}^{3}$ (baseline), 13 features (1 tumor intensity and 12 wavelet) were found to be significantly $(P<0.05)$ different. The second CT was acquired 4 days after baseline, the day animals were sham irradiated, and only 1 wavelet feature was observed to be different. At the third CT, at the final volume of $500 \mathrm{~mm}^{3}$, 5 features ( 1 LoG and 4 wavelets) were significantly $(P<0.05)$ different (Figure 4). However, these features were different across the three CT image time points. None of the features identified were correlated with tumor volume.

After RT (second CT acquisition), 17 features (3 tumor intensity and 15 wavelets) differed significantly $(P<0.05)$ between the dox+ group and doxgroup. Five of those 17 features differed significantly $(P<0.05)$ between irradiated (RT+) and sham-irradiated (RT-) animals within the dox-group ( 3 tumor intensity and 2 wavelet). One feature $(P<0.05)$ (wavelet) differed between RT+ and RT- within the doxycycline-treated group (supplementary table 1). These 6 features are related to early effect of RT alone. At the final image acquisition time point, 8 features (wavelet) differed significantly $(P<0.05)$ between the dox+ and dox-groups. One of these 8 was found to be significantly different between RT+ vs RT- within dox-group derived at final time point. The remaining 7 features were 
consistent, since they were statistically different between the dox+ and dox-groups at both time points, 4 days post RT and at the final time point . These features were specific for the combination of RT with change in GADD34 gene expression (Figure 4). Features found to be significantly different at all assessed time points after irradiation between dox+ and dox- groups were always consistently higher for dox+. An example is shown in Figure 5A.

To further validate our findings, we obtained features from CTs performed at a higher energy level (80kVp) (Figure 3). At baseline, 9 features ( 1 tumor intensity, 1 gray-level intensity and 7 wavelet) were found to differ significantly between dox+ and dox- treatment groups. Similar to $40 \mathrm{kVp}$, these features lost significance at later time points. Upon RT, 9 features (wavelet) differed significantly between dox+ and dox-. Five of those features were also found in the $40 \mathrm{kVp} C \mathrm{CT}$ images and out of which, one feature $(P<0.05)$ (wavelet) significantly differed between RT+ and RT- within the dox-group (supplementary table 2). The remaining 4 image features were also significantly different at later time points, but were not identified in the $40 \mathrm{kVp}$ images (Figure 4) (Supplementary Table 2). Similar to the $40 \mathrm{kVp}$ obtained features, the $80 \mathrm{kVp}$ image features were also consistently higher for dox+ than dox- at both imaging time points (Figure 5B) 


\section{Discussion:}

Radiomics, an advanced analysis platform, extracts features from medical images by using mathematical algorithms based on tumor intensity, graylevel intensities, and texture [6-9,15,18-21]. We have previously identified radiomics-based signatures that are prognostic and associated with proliferation-related gene signatures [7]. Furthermore, radiogenomics imaging traits might act as molecular surrogates for predicting treatment responses [11]. The studies conducted so far on radiomics have only shown correlative association of radiomic signatures with gene signatures. In this preclinical radiogenomics study, we hypothesized that image features are causally related to genetic factors and that radiation affects these image features.

To prove our hypothesis, we first employed a genetic tumor model in which tumor microenvironmental characteristics like hypoxia change upon doxycycline administration[12]. We have previously demonstrated that tumoral GADD34 overexpression led to an inhibition of the elF2 $\alpha$ signaling pathway upon administration of doxycycline, resulting in decreased hypoxia tolerance and as a result reduced hypoxic fraction. Although this had no effect on tumor growth, a significantly enhanced growth delay was observed upon combination with tumor irradiation when compared with RT monotherapy [12]. Thus by employing this reproducible model under well-controlled experimental conditions, we could identify whether a change in GADD34 gene expression, is causally related with radiomics image features and whether this could be affected by radiation.

We have observed that a genetic change (dox+ vs dox- treatment group) results in significantly different radiomics features, although these features were not consistent across the different imaging time points. Next, certain radiomics features were significantly different upon RT monotherapy (RT- vs RT+ dox- treatment groups). Similar to genetic changes, features that differed significantly upon irradiation were not consistent between different CT acquisitions. This can be explained due to the dynamic changes in the tumor and tumor microenvironment over time. Along with tumor growth, necrotic fraction may also increase and hypoxic fraction may change. Post radiation therapy, neovasculature is formed; hypoxic repopulation occurs leading to intra-tumoral heterogeneity over time [22].

However, when GADD34 was induced and radiation therapy was given, there was additional growth delay due to a decreased hypoxic fraction at 
the time of irradiation, as previously described [12]. Thus the difference in tumor heterogeneity between gene-induced and non-induced tumors post RT was reflected in the imaging features likely as a result of a phenotypic change. Interestingly, the radiomics image features that were found to be significantly different between both groups shortly after irradiation were also observed at larger tumor volumes. This phenomenon was observed independent of the CT image acquisition energy level, although the observed features were different between both energies tested. Remarkably, the feature value for slow-growing tumors (gene-induced) was higher than for faster-growing tumors (no gene-induced group) upon combination with radiotherapy. 
Conclusions: We have shown in in vivo preclinical models that radiomics is able to quantify the early effects of radiation treatment and genetic changes in tumors with similar volumes, and identify differences that are not visible to the human eye.

\section{Acknowledgements}

The authors acknowledge the support of the QulC-ConCePT project, partly funded by EFPIA companies and the Innovative Medicine Initiative Joint Undertaking (IMI JU) under Grant Agreement No. 115151. The authors also acknowledge financial support from the National Institute of Health (NIH-USA U01 Radiomics), the EU 6th and 7th framework program (ARTFORCE) and Kankeronderzoekfonds Limburg from the Health Foundation.

\section{Conflict of interest}

All authors declare that there are no conflicts of interest. 
Figure legends:

Figure 1: Graphical representation of experimental work flow

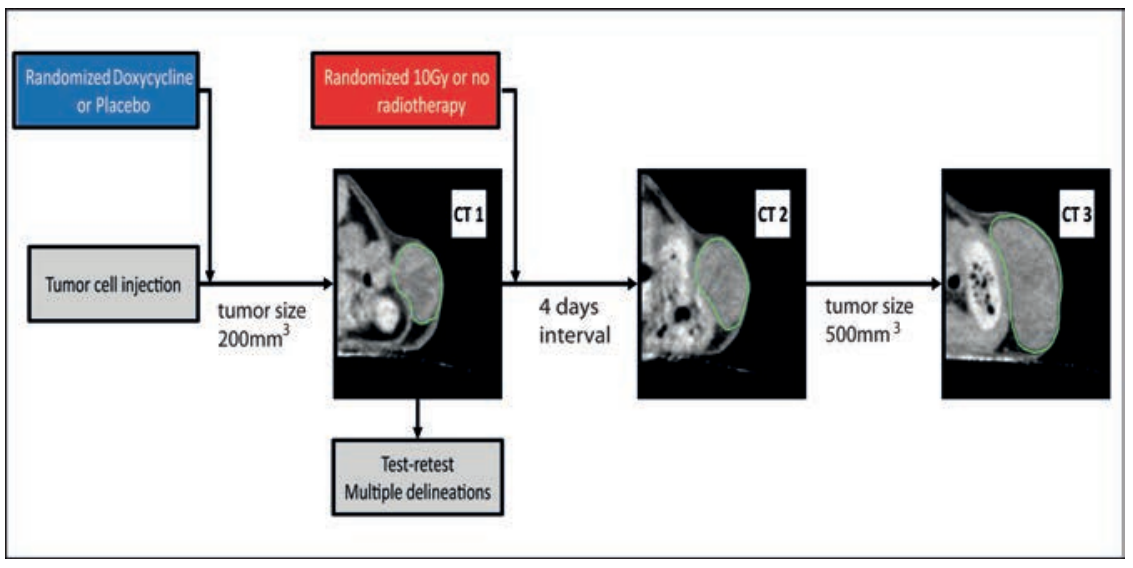

Figure 2: Time to reach 4 times start volume (T4xSV) for the different treatment groups. $* P<0.05, * * P<0.01$. Data represent the mean \pm SD of at least 6 animals.

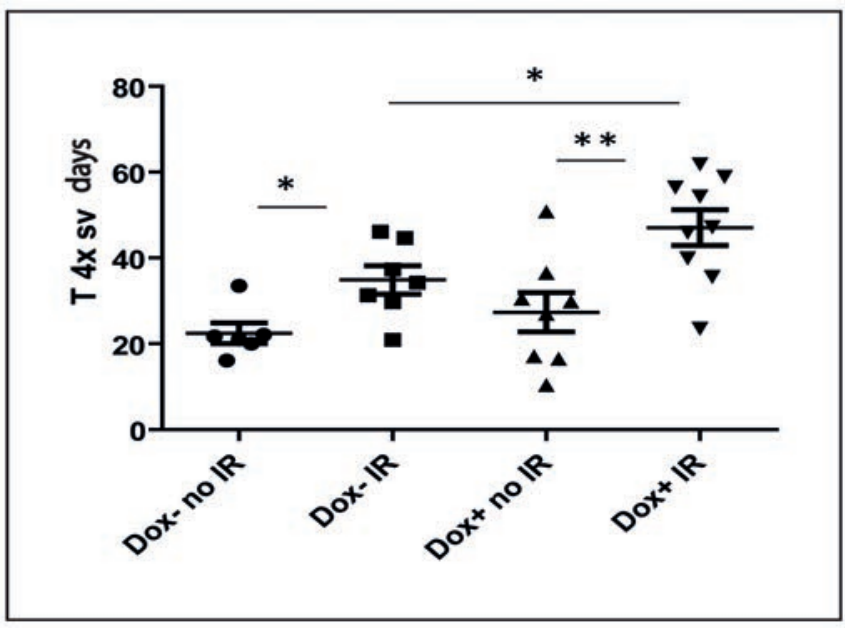


Figure 3: Representative images of $40 \mathrm{kVp}$ and $80 \mathrm{kVp}$ energy $\mathrm{CT}$ and feature selection based on average ICC values of test-retest and inter-observer variability.

$40 k v p$ CT scan

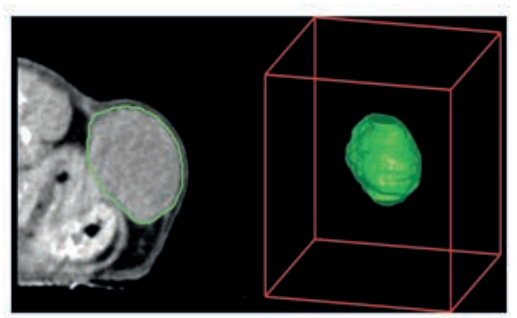

80kvp CT scan

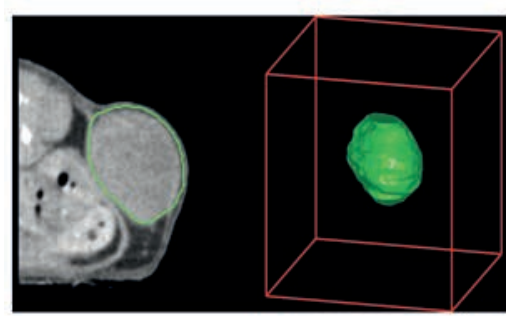

Test-retest ICC vs Inter-observer ICC 40kvp

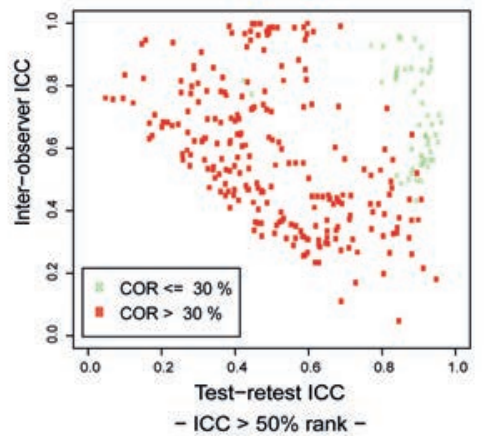

Test-retest ICC vs Inter-observer ICC 80kvp

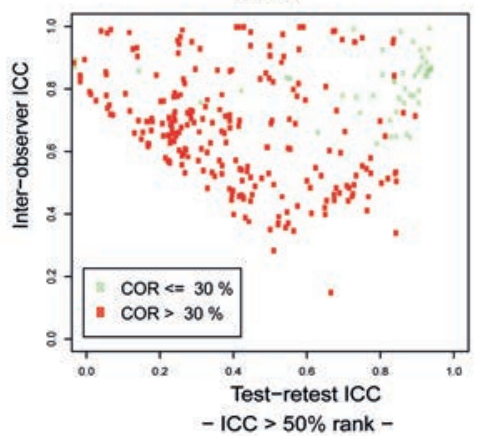


Figure 4: Schematic representation of CT scan derived radiomic features (40kVp purple and $80 \mathrm{kV}$ green) for different treatment groups at different time points. The numbers within the arrows show the number of features, which are consistent between the indicated imaging time points and different energies.

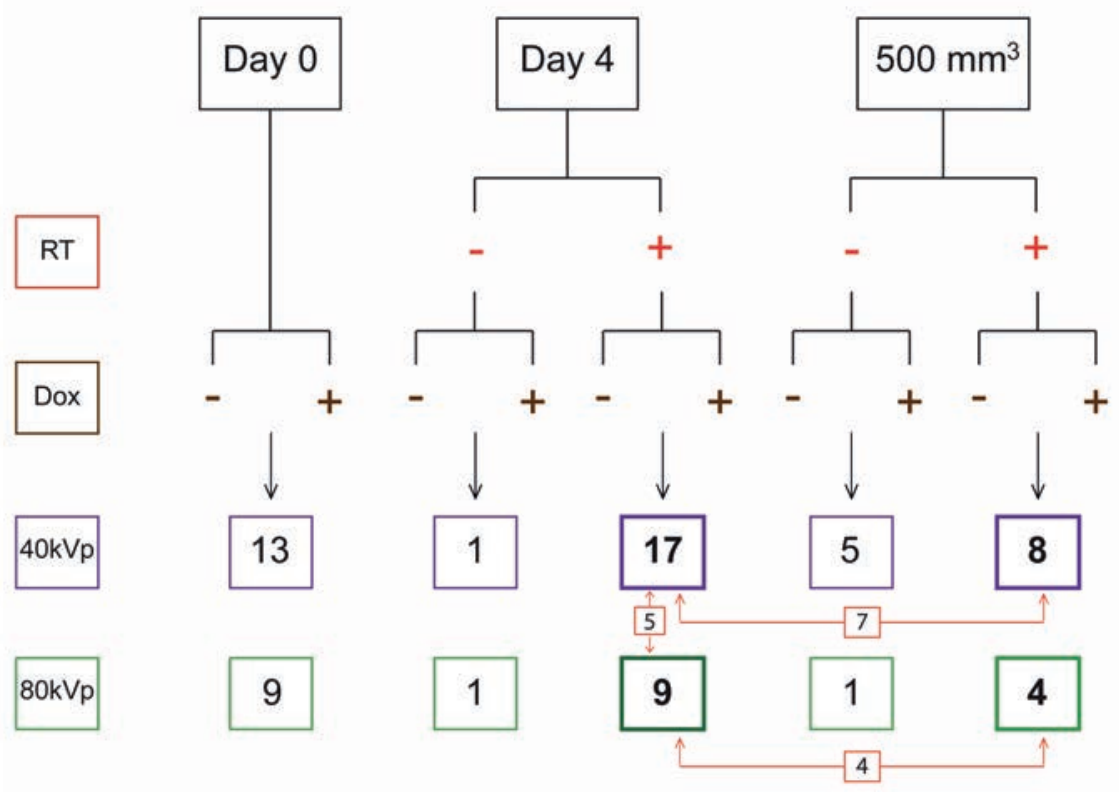


Figure 5: Gene induction (dox+) results in significantly changed features compared to no gene induction (dox-) upon combination with radiotherapy 4 days after RT and at $500 \mathrm{~mm} 3$. Features are extracted from $40 \mathrm{kVp}(\mathrm{A})$ or $80 \mathrm{kVp}$ (B) CT scans.

4 day post irradiation

$5 \mathrm{~A}$

Wavelet_LHH_stats_rms,$p=0.016$

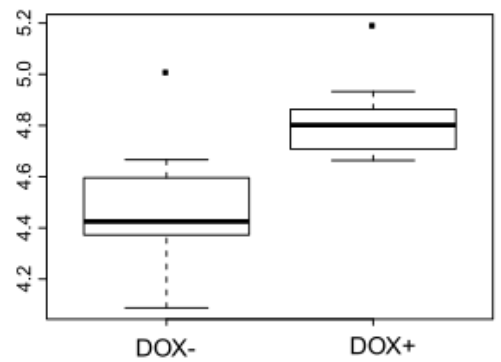

$5 B$

Tumor size of $500 \mathrm{~mm} 3$

Wavelet_LHH_stats_rms, $p=0.032$

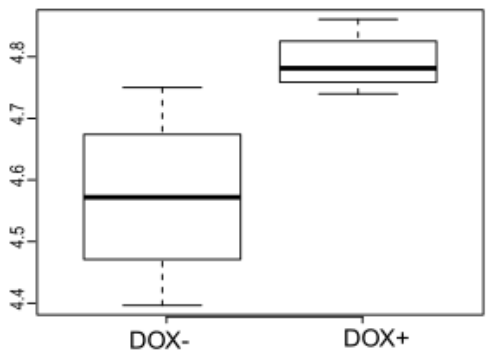

Wavelet_HHH_stats_md, $p=0.016$

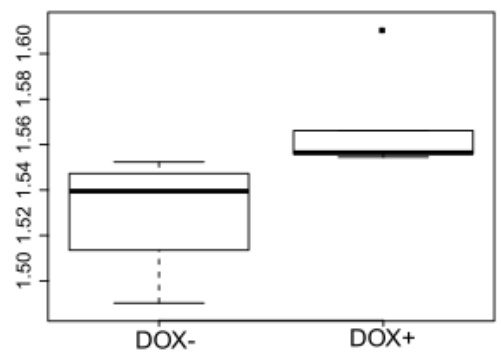


Supplementary Table 1: Features derived from $40 \mathrm{kVp}$ that are found in various groups at early ( 4 days post) and final CT $\left(500 \mathrm{~mm}^{3}\right)$. Consistent features that are significantly different between dox+ and dox- post irradiation at both time points are highlighted in purple. At final $C T$, no features were present in the dox+ RT+ vs RT- compared with the other groups.

\begin{tabular}{|c|c|c|c|c|c|c|}
\hline \multirow[b]{2}{*}{ Features } & \multicolumn{3}{|c|}{4 days post } & \multicolumn{3}{|c|}{ final CT $\left(500 \mathrm{~mm}^{3}\right)$} \\
\hline & $\begin{array}{c}\text { RT+ } \\
\text { dox+ } \\
\text { vs } \\
\text { dox- }\end{array}$ & $\begin{array}{c}\text { dox- } \\
\text { RT+ vs } \\
\text { RT- }\end{array}$ & $\begin{array}{c}\text { dox+ } \\
\text { RT+ vs } \\
\text { RT- }\end{array}$ & $\begin{array}{c}\text { RT+ } \\
\text { dox+ } \\
\text { vs } \\
\text { dox- }\end{array}$ & $\begin{array}{c}\text { dox- } \\
\text { RT+ vs } \\
\text { RT- }\end{array}$ & $\begin{array}{c}\text { dox+ } \\
\text { RT+ vs } \\
\text { RT- }\end{array}$ \\
\hline Stats_max & 0.042 & 0.035 & & & & \\
\hline Wavelet_HLH_stats_md & 0.016 & & & 0.016 & 0.016 & \\
\hline Stats_mean & 0.042 & 0.005 & & & & \\
\hline Stats_rms & 0.042 & 0.005 & & & & \\
\hline Wavelet_LLL_stats_mean & 0.042 & 0.005 & & & & \\
\hline $\begin{array}{l}\text { Wavelet_LLL_stats_rms } \\
\text { Wavelet_LHH_glcm_infoCor }\end{array}$ & 0.042 & 0.005 & & & & \\
\hline r2 & 0.005 & & 0.008 & & & \\
\hline Wavelet_HLH_stats_rms & 0.012 & & & 0.016 & & \\
\hline Wavelet_HLH_stats_std & 0.012 & & & 0.016 & & \\
\hline Wavelet_HLH_stats_var & 0.012 & & & 0.016 & & \\
\hline Wavelet_LHH_stats_md & 0.016 & & & 0.032 & & \\
\hline Wavelet_LHH_stats_rms & 0.016 & & & 0.032 & & \\
\hline Wavelet_LHH_stats_std & 0.016 & & & 0.032 & & \\
\hline Wavelet_LHH_stats_var & 0.016 & & & 0.032 & & \\
\hline Wavelet_LHH_stats_max & 0.016 & & & & & \\
\hline Wavelet_LHH_stats_range & 0.042 & & & & & \\
\hline Wavelet_LLH_stats_md & 0.042 & & & & & \\
\hline
\end{tabular}


Supplementary Table 2: Consistent features (in green) derived from $80 \mathrm{kVp}$ CT at early (4 days post) and late time points post RT (tumor volume of $500 \mathrm{~mm}^{3}$ ). In red are features that were also found in the $40 \mathrm{kVp}$ scan ( 4 days post RT). One feature was also found within doxRT+ vs RT- comparison.

\begin{tabular}{|c|c|c|c|}
\hline Features & $\begin{array}{c}4 \text { days post } \\
\text { RT+ dox+ vs dox- }\end{array}$ & $\begin{array}{c}500 \mathrm{~mm}^{3} \\
\mathrm{RT}+\text { dox+ vs dox- }\end{array}$ & $\begin{array}{c}4 \text { days post } \\
\text { dox- RT+ vs RT- }\end{array}$ \\
\hline Wavelet_HHH_stats_md & 0.042 & 0.016 & \\
\hline Wavelet_HHH_stats_rms & 0.031 & 0.016 & \\
\hline Wavelet_HHH_stats_std & 0.031 & 0.016 & \\
\hline Wavelet_HHH_stats_var & 0.031 & 0.016 & \\
\hline Wavelet_LHH_stats_md & 0.031 & & \\
\hline Wavelet_LHH_stats_rms & 0.031 & & \\
\hline Wavelet_LHH_stats_std & 0.031 & & \\
\hline Wavelet_LHH_stats_var & 0.031 & & \\
\hline Wavelet_LLH_stats_md & 0.031 & & 0.031 \\
\hline
\end{tabular}




\section{References}

[1] Lambin P, van Stiphout RGPM, Starmans MHW, et al. Predicting outcomes in radiation oncology[mdash]multifactorial decision support systems. Nat Rev Clin Oncol 2013;10:27-40.

[2] Chin L, Andersen JN Futreal PA. Cancer genomics: from discovery science to personalized medicine. Nat Med 2011;17:297-303.

[3] Lee S, de Boer WB, Fermoyle S, Platten M Kumarasinghe MP. Human epidermal growth factor receptor 2 testing in gastric carcinoma: issues related to heterogeneity in biopsies and resections. Histopathology 2011;59:832-840.

[4] Ruijter ET, Van De Kaa CA, Schalken JA, Debruyne FM Ruiter DJ. Histological grade heterogeneity in multifocal prostate cancer. Biological and clinical implications. The Journal of Pathology 1996;180:295-299.

[5] Rudin M Weissleder R. Molecular imaging in drug discovery and development. Nat Rev Drug Discov 2003;2:123-131.

[6] Lambin P, Rios-Velazquez E, Leijenaar R, et al. Radiomics: Extracting more information from medical images using advanced feature analysis. European Journal of Cancer 2012;48:441-446.

[7] Aerts HJWL, Velazquez ER, Leijenaar RTH, et al. Decoding tumour phenotype by noninvasive imaging using a quantitative radiomics approach. Nat Commun 2014;5.

[8] Velazquez ER, Parmar C, Jermoumi M, et al. Volumetric CT-based segmentation of NSCLC using 3D-Slicer. Sci. Rep. 2013;3.

[9] Coroller TP, Grossmann P, Hou Y, et al. CT-based radiomic signature predicts distant metastasis in lung adenocarcinoma. Radiotherapy and Oncology 2015;114:345-350.

[10] Segal E, Sirlin CB, Ooi C, et al. Decoding global gene expression programs in liver cancer by noninvasive imaging. Nat Biotech 2007;25:675-680.

[11] Kuo MD, Gollub J, Sirlin CB, Ooi C Chen X. Radiogenomic analysis to identify imaging phenotypes associated with drug response gene expression programs in hepatocellular carcinoma. Journal of Vascular and Interventional Radiology 2007;18:821-830.

[12] Rouschop KM, Dubois L, Keulers TG, et al. PERK/elF2 $\alpha$ signaling protects therapy resistant hypoxic cells through induction of glutathione synthesis and protection against ROS. Proceedings of the National Academy of Sciences 2013;110:46224627.

[13] van Hoof SJ, Granton PV Verhaegen F. Development and validation of a treatment planning system for small animal radiotherapy: SmART-Plan. Radiotherapy and Oncology 2013;109:361-366.

[14] Deasy JO, Blanco Al Clark VH. CERR: A computational environment for radiotherapy research. Medical Physics 2003;30:979-985.

[15] Leijenaar RTH, Carvalho S, Velazquez ER, et al. Stability of FDG-PET Radiomics features: An integrated analysis of test-retest and inter-observer variability. Acta Oncologica 2013;52:1391-1397.

[16] Shrout PE Fleiss JL. Intraclass correlations: Uses in assessing rater reliability. Psychological Bulletin 1979;86:420-428.

[17] Bland JM Altman DG. Agreement Between Methods of Measurement with Multiple Observations Per Individual. Journal of Biopharmaceutical Statistics 2007;17:571-582.

[18] Chicklore S, Goh V, Siddique M, Roy A, Marsden P Cook GR. Quantifying tumour heterogeneity in 18F-FDG PET/CT imaging by texture analysis. European Journal of Nuclear Medicine and Molecular Imaging 2013;40:133-140. 
[19] Parmar C, Rios Velazquez E, Leijenaar R, et al. Robust Radiomics Feature Quantification Using Semiautomatic Volumetric Segmentation. PLoS ONE 2014;9:e102107.

[20] Kumar V, Gu Y, Basu S, et al. Radiomics: the process and the challenges. Magnetic Resonance Imaging 2012;30:1234-1248.

[21] Carvalho S, Leijenaar RTH, Velazquez ER, et al. Prognostic value of metabolic metrics extracted from baseline positron emission tomography images in nonsmall cell lung cancer. Acta Oncologica 2013;52:1398-1404.

[22] Moulder J Rockwell S. Tumor hypoxia: its impact on cancer therapy. Cancer and Metastasis Reviews 1987;5:313-341. 


\section{Chapter 6}

Radiomics approach to evaluate tumor hypoxia on CT

Manuscript in preparation

Panth KM, Carvalho S, Leijenaar RTH, Peeters SGJA, Even A, Oberije C, Zegers CML, Reymen B, Van Elmpt WJ, Yaromina A, Dubois LJ, Lambin P 


\begin{abstract}
Radiomics, an advanced image analysis methodology, might reflect underlying tumor heterogeneities. In this study we hypothesized that computed tomography (CT) image derived radiomics features reflects tumoral oxygenation status. For this purpose, first the effect of oxygen modification on CT-derived radiomics features was studied in two independent datasets using rat rhabdomyosarcoma tumors. In both datasets, low oxygen breathing (7\%) caused significant changes in 15 features compared to control (21\% Oxygen), of which 6 features correlated with the uptake of the hypoxia PET tracer $\left[{ }^{18} \mathrm{~F}\right]-\mathrm{HX} 4$. Next, the effect on radiomics features of hypoxia-targeted treatments was studied using a hypoxia activated pro-drug ( $\mathrm{TH} 302)$ in rats and a vasodilator (nitroglycerin) in lung cancer patients. Six features that were found in both preclinical and clinical datasets correlated with $\left[{ }^{18} \mathrm{~F}\right]-\mathrm{HX} 4$ uptake. A wavelet feature strongly correlated with the hypoxic fraction $(R=0.6425$ and $R=0.7059$ for rats and patients, respectively) and hypoxic volume ( $R=$ 0.6013 and $R=0.7927$ for rats and patients respectively) in both datasets. In conclusion, CT-derived radiomics features correlated with $\left[{ }^{18} \mathrm{~F}\right]-\mathrm{HX} 4$ uptake in both rat tumors and human lung cancer patients. Therefore, CTderived radiomics offers a promising non-radioactive method for hypoxia imaging.
\end{abstract}




\section{Introduction}

Tumor hypoxia is associated with poor prognosis and radioresistance [13]. Several diagnostic and therapeutic compounds are being developed to visualize and target hypoxia. Noninvasive imaging of hypoxia enables detection of treatment resistant phenotypes and monitoring treatment efficacy [4]. One of the well described positron emission tomography (PET) tracers for detecting hypoxia is flortanidazole $\left(\left[{ }^{18} \mathrm{~F}\right]-\mathrm{HX} 4\right)$ [5-13]. It was demonstrated that $\left[{ }^{18} \mathrm{~F}\right]-\mathrm{HX} 4$ uptake in tumors spatially correlated with hypoxia marker pimonidazole [6]. Furthermore, the $\left[{ }^{18} \mathrm{~F}\right]-\mathrm{HX} 4$ measured hypoxic fraction before treatment with a hypoxia activated pro-drug (TH302) that selectively kills hypoxic cells was predictive for its treatment efficacy [13]. However, the accessibility of the tracer is limited due to constraining factors such as time, cost and required facilities for the production of the tracer.

Computed Tomography (CT) imaging is routinely used in clinical practice for cancer diagnosis and treatment planning. In addition to shape and size features, intratumoral heterogeneities are hypothesized to be hidden in CT images and possibly decoded by imaging features [14]. Radiomics is an advanced image analysis methodology that extracts a large number of quantitative features from medical images and has been proven to be prognostic in cancer studies [14-25] (http://www.radiomics.org, animation: https://youtu.be/VfOF7q8vaS4). Radiomics is currently an active field of research and its potential for clinical application is increasingly reported. Recently, our group also demonstrated a causal link between gene change and CT-derived imaging features in a preclinical setting [22].

In the current study, we hypothesized that tumor microenvironment characteristics, namely hypoxia can be inferred from CT-derived radiomics features. For this, it is essential to obtain good understanding about the functional relationship of these features and the underlying intratumoral hypoxia status. For this purpose, the change in radiomics features due to low oxygen (7\%) breathing was first studied in two independent datasets using the rat rhabdomyosarcoma tumor model previously described $[9,13]$. Next, the impact of a change in tumor oxygenation on radiomics features as a result of hypoxia activated prodrug TH302 treatment was evaluated in rat rhabdomyosarcoma tumors. These features were validated in human lung cancer patients who received a nitroglycerin patch (a vasodilator) prior to radiation therapy (NCT01210378). Although, TH302 and Nitroglycerin exhibit different mechanisms for the reduction of hypoxia, both treatments are aimed 
to reduce hypoxic fraction in the tumors. Since $\left[{ }^{18} \mathrm{~F}\right]-\mathrm{HX} 4$ is an established hypoxia biomarker, radiomics features obtained due to low oxygen (7\%) breathing or due to hypoxia targeting treatments were compared to the $\left[{ }^{18} \mathrm{~F}\right]-\mathrm{HX} 4$ uptake in these tumors. Therefore, the aim of the current study is to provide a proof-of-concept that tumor hypoxia can be deciphered by radiomics features.

\section{Methods}

Oxygen modification features: Oxygen modification was performed in two independent experiments in rat rhabdomyosarcoma tumors that were previously described in detail $[9,13]$. In the first dataset, rats were subjected to either low oxygen $(7 \%)(n=31)$ or normal $(21 \%)(n=32)$ breathing for 3 hours [9]. $\left[{ }^{18} \mathrm{~F}\right]-\mathrm{HX} 4$ PET/CT imaging was performed (in $\mathrm{n}=$ 10 rats) before and after oxygen modification using a clinical PET/CT scanner (Siemens Biograph 40; Siemens Healthcare). Radiomics features were extracted from both pre and post oxygen modification CT images. Radiomics features that significantly differed between pre and post oxygen modification were further validated in a second dataset, in which rats were subjected to 4 hours of $7 \%(n=30)$ or $21 \%(n=43)$ oxygen breathing followed by $\left[{ }^{18} \mathrm{~F}\right]-\mathrm{HX} 4 \mathrm{PET} / \mathrm{CT}$ imaging [13]. The $\left[{ }^{18} \mathrm{~F}\right]-\mathrm{HX} 4$ tracer was injected in between the oxygen modification i.e., oxygen modification was done 2 hours before and 2 hours after tracer injection. $\left[{ }^{18} \mathrm{~F}\right]-\mathrm{HX} 4 \mathrm{PET} / \mathrm{CT}$ images were acquired ( $n=30$ rats) on the same clinical scanner as described in the first dataset. A significant increase in the uptake of $\left[{ }^{18} \mathrm{~F}\right]-\mathrm{HX} 4$ was demonstrated with $7 \%$ oxygen breathing compared to $21 \%$ oxygen breathing in both datasets $[9,13]$. A schematic representation of the oxygen modification and $\left[{ }^{18} \mathrm{~F}\right]-\mathrm{HX} 4$ PET/CT acquisition for both datasets is shown in Supplementary figure 1. Significantly different features that were found in both datasets were further compared to mean standardized uptake values (SUV) and tumor to background ratios (TBR) obtained from $\left[{ }^{18} \mathrm{~F}\right]-\mathrm{HX} 4$ PET images.

Hypoxia-treatment related features: In a previously described dataset, rat rhabdomyosarcoma tumors were treated with TH302 $(25 \mathrm{mg} / \mathrm{kg})$ $(n=6)$ or saline $(n=6)$ for 4 consecutive days [13]. $\left[{ }^{18} \mathrm{~F}\right]-\mathrm{HX} 4$ PET/CT imaging was performed both at baseline and post treatment on a clinical scanner (Siemens Biograph 40, Siemens Healthcare). The hypoxic fraction in the TH302 treated group decreased on average from $23 \%$ to $2.5 \%$ while the hypoxic fraction in the control group remained around $21 \%$. Radiomics features were extracted from CT images acquired at day 4 in both saline and TH302 treated groups. Features that significantly differed between saline and TH302 treatment were further validated in non-small cell lung 
cancer (NSCLC) patients who received a standard low dose nitroglycerin patch for 12 hours daily before radiotherapy (NCT01210378) ( $n=12) .\left[{ }^{18} \mathrm{~F}\right]-$ HX4 PET/CT images were acquired prior and post nitroglycerin treatment. The median interval between the baseline and nitroglycerin scans was 4.5 days (range 2-7 days). Nitroglycerin patch was applied 2 hours before $\left[{ }^{18} \mathrm{~F}\right]-\mathrm{HX} 4$ injection for the latter scan. Radiomics features were extracted from both pre and post nitroglycerin treatment. Significantly different features that were found in both preclinical and clinical datasets were compared to SUVmean, TBR, hypoxic fractions (HF) and volumes derived from $\left[{ }^{18} \mathrm{~F}\right]-\mathrm{HX} 4$ PET images. Tumor areas with a TBR (SUV mean in heart as background) larger than 4.5 were assumed hypoxic in rat dataset and TBR (with SUVmean in the aorta as background) above 1.4 were assumed to be hypoxic in patient dataset. Hypoxic volume (HV) was obtained by multiplying the hypoxic fraction with the tumor volume. The Nitroglycerin trial (NCT01210378) was approved by the Medical Ethics Review Committee. All animal experiments were approved and were in accordance with Animal Ethical Committee of Maastricht University.

Radiomics analysis: Radiomics features were extracted from CT images using in-house developed radiomics software. A total of 723 features were extracted from each image, consisting of five main groups: 1) first order statistics, 2) shape and size, 3) texture descriptors including gray level co-occurrence (GLCM), gray level run-length (GLRLM) and gray level size-zone texture matrices (GLSZM), 4) features from groups 1 and 3 after wavelet decomposition and 5) features derived from Group 1 after applying a Lapacian of Gaussian (LOG). The definitions and detailed description of features are described elsewhere $[17,18,20]$. Two baseline scans obtained before treatment in rat rhabdomyosarcoma tumors were used as test-retest (TRT) [9] and used to eliminate features with low repeatability.

Statistical analysis: Wilcoxon signed-rank test or Wilcoxon rank-sum test were used to compare statistical differences between paired or unpaired groups, respectively. $\mathrm{P}$ values were not corrected for multiple testing. Permutation test was performed for determining the probability of random overlap between respective groups. Linear regression analysis and Pearson correlations were performed using GraphPad prism 5.0 to compare radiomics features with $\left[{ }^{18} \mathrm{~F}\right]-\mathrm{HX} 4$ PET uptake. A P-value was considered significant at a 0.05 level. 


\section{Results}

\section{Radiomics features related to oxygen modification}

A total of 156 features (4 first order statistics, 3 shape and size, 11 texture, 86 wavelet and 52 LoG) significantly differed between test-retest (TRT) control CT images, which were eliminated from further analysis. From the remaining features, 39 ( 5 first order statistics, 6 shape and size, 17 wavelet and 11 LoG) significantly differed between baseline and 3 hour post normal $\left(\begin{array}{lll}21 \% & \mathrm{O}_{2}\end{array}\right)$ breathing. Because these features are independent of oxygenation status, they were excluded from further analysis. Between baseline and 3 hours low oxygen $\left(7 \% \mathrm{O}_{2}\right)$ breathing 74 significantly different features ( 3 first order statistics, 5 shape and size, 2 texture, 44 wavelet and 20 LoG ) were found, of which 63 features (1 first order statistics, 2 shape and size, 2 texture, 38 wavelet and 20 LoG) exclusively belonged to $7 \% \mathrm{O}_{2}$ breathing (Figure 1). As a validation, we determined differences between $21 \%$ and $7 \%$ breathing in the second dataset. After TRT control feature elimination, a total of 121 features ( 2 first order statistics, 5 shape and size, 4 texture, 33 wavelet and 77 LoG) significantly differed between $21 \% \mathrm{O}_{2}$ and $7 \% \mathrm{O}_{2}$ breathing conditions. 15 features ( 2 shape, 2 wavelet and 11 LoG) were found in both datasets (Figure 1), and the probability of finding 15 common features between the datasets was found to be low $(P=0.0799)$. These features were further compared to $\left[{ }^{18} \mathrm{~F}\right]-\mathrm{HX} 4$ PET uptake in the tumors (Supplementary table 1 ). 6 features correlated with the HX4 uptake in both datasets. For example, LoG sigma $10 \mathrm{~mm}$ 3D first order statistics entropy showed a significant positive correlation and LoG sigma $10 \mathrm{~mm}$ 3D first order statistics uniformity showed a negative correlation with SUVmean after $7 \% \mathrm{O}_{2}$ breathing in both datasets (Figure 2A). In line with these correlations, these features significantly increased or decreased after $7 \% \mathrm{O}_{2}$ as compared with $21 \% \mathrm{O}_{2}$ breathing (Figure $2 \mathrm{~B}$ ).

\section{Radiomics features related to hypoxia targeting}

A total of 240 features (7 first order statistics, 21 texture, 208 wavelet and 4 LoG) significantly differed between saline and TH302 treatment in the preclinical dataset. 204 features ( 5 first order statistics, 16 texture, 181 wavelet and 2 LOG) remained after TRT control elimination (Figure $3 \mathrm{~A})$. As a validation, we evaluated the differences in features between pre and post nitroglycerin treatment in the clinical dataset. 24 features $(9$ texture, 14 wavelet and $1 \mathrm{LOG}$ ) were found to be significantly different in the clinical dataset (Figure 3A). Six features (5 texture and 1 wavelet) were found to be consistent in both the preclinical and clinical datasets 
(Figure 3A). However, the probability of finding 6 overlapping features between the groups was found to be high $(P=0.5325)$. Nevertheless, the features correlated with HX4 uptake quantified as SUVmean, TBR, HF and HV (Supplementary table 2 and Figure 3B). As an example, the wavelet LLL gray level size zone variability correlated significantly with the hypoxic fraction in both the preclinical and clinical dataset $(R=0.6425$ and $R=$ 0.7059 for rats and patients respectively) (supplementary table 2 and Figure 4A). The same wavelet feature also highly correlated with hypoxic volume in both the datasets $(R=0.6013$ and $R=0.7927$ for rats and patients respectively) (Figure 4B). 


\section{Discussion}

Tumor hypoxia is associated with worse prognosis and poor treatment outcome $[2,3,26]$. Imaging hypoxia before and after treatment targeting hypoxia can aid in patient selection and monitoring treatment response [1]. However, the availability of a PET tracer for detecting hypoxia can be limited due to factors such as time, cost and adequate facilities for the production of tracer. CT imaging is widely used in the clinic for detecting tumors and planning of radiation treatment. In this proof-of-concept study, we aimed 1) to identify radiomics features corresponding to low oxygenation of tumors and 2) to evaluate the features that can predict tumor hypoxia in preclinical and clinical setting.

We have observed that 11 out of 15 features identified due to temporary changes in oxygen were found to be LoG features derived from first order statistics, indicating that gray level intensity values were the most affected. Furthermore, an increase in feature value was observed for most of the features similar to increase in $\left[{ }^{18} \mathrm{~F}\right]-\mathrm{HX} 4$ PET uptake after $7 \%$ oxygen breathing. On the other hand, two LoG features derived from first order uniformity showed a negative correlation with $\left[{ }^{18} \mathrm{~F}\right]-\mathrm{HX} 4 \mathrm{PET}$ uptake and decreased after $7 \% \mathrm{O}_{2}$, reflecting decreased pixel intensity uniformity and increased intratumoral heterogeneity. Altogether, this indicates that the radiomics features were able to detect oxygen change in tumors similar to hypoxia PET tracer. These findings suggest that radiomics features might be used to evaluate tumor oxygenation status during or after radiotherapy, which warrants further investigations.

We also assessed the effect of hypoxia-targeted treatment in rat rhabdomyosarcoma tumors and in lung cancer patients. Since previously it was demonstrated that $\mathrm{TH} 302$ was effective in reducing hypoxic fraction, we first identified features that were altered upon TH302 treatment. Nitroglycerin, a vasodilator is also aimed to reduce hypoxic fraction although the mechanism of action differs from TH302. Therefore, features that were similarly affected in both preclinical and clinical datasets (TH302 and nitroglycerin) were assumed to be due to hypoxiatargeted treatment and therefore were compared to $\left[{ }^{18} \mathrm{~F}\right]-\mathrm{HX} 4$ PET tracer uptake. A low number of features significantly differed between pre and post nitroglycerine treatment in patient dataset ( 24 features) compared to preclinical dataset (204 features). This can be expected since not all patients showed a reduced hypoxia with nitroglycerin treatment. Only 5 patients showed a reduced hypoxia while, 1 patient showed no change in hypoxia and 6 patients showed no hypoxic fraction before and after treatment. While with $\mathrm{TH} 302$ treatment in the preclinical dataset hypoxia 
was significantly reduced in all TH302 treated rats. However, features that were found in both datasets correlated with SUVmean or TBR derived from $\left[{ }^{18} \mathrm{~F}\right]-\mathrm{HX} 4$ PET. A textural feature after wavelet decomposition (wavelet LLL gray level size zone variability) highly correlated with the hypoxic fraction and the hypoxic volume in both datasets. A reduction in the feature value was observed with TH302 treatment (Supplementary figure 2A) and this effect was independent of tumor volume as there was no correlation found with tumor volume in rat rhabdomyosarcoma tumors (Supplementary figure 2B). Wavelet LLL gray level size zone variability is derived from textural features (Gray level size zone matrix features) which describes the heterogeneity of the tumor at a regional level $[17,20]$. Therefore, a decrease in the feature value with TH302 treatment can be related to the reduction in heterogeneity of the tumor. It was previously reported that the hypoxic volume might be a better prognostic marker as it represents the number of hypoxic cells in a certain volume. A larger tumor might contain lesser number of hypoxic cells and vice versa [27]. Although, 4 out of 6 features correlated with hypoxic fraction and hypoxic volume in the rat rhabdomyosarcoma, this correlation could be observed for patients in only one feature. One possible explanation is that the hypoxic fraction in the patients was relatively low (10 $\pm 15 \%)$ whereas, rat rhabdomyosarcoma tumors are known to have large hypoxic fractions [28]. Furthermore, the hypoxic fractions were calculated based on different TBR thresholds. Despite of these factors, this radiomics feature highly correlated in both datasets with $\left[{ }^{18} \mathrm{~F}\right]-\mathrm{HX} 4$ uptake. The features however could not be validated for predictive power for treatment response due to limited data availability. Preclinical radiomics studies has the advantage of understanding a known effect on imaging features and aids in selection of right features by use of proper control which is impractical in clinical setting. However there are certain limitations in using preclinical datasets that have small group sizes while radiomics studies usually requires large datasets [15]. Furthermore, due to large number of features the chance of finding significant and overlapping features between the groups is high. Nevertheless, use of proper control and test retest methods for selecting features reduces the chance of random findings. Furthermore, similar behavior of features in two independent datasets irrespective of different settings makes these features reliable.

In this proof-of-concept study, we demonstrated that hypoxia and temporary change in oxygen influence radiomics features. These features correlate to hypoxia $\left[{ }^{18} \mathrm{~F}\right]-\mathrm{HX} 4$ PET tracer uptake and therefore have a potential to be applied for monitoring of tumoral oxygenation status 
during/after hypoxia targeted therapies. However, this requires additional studies in both preclinical and clinical setting.

\section{Conclusions}

In this proof-of-concept study, we demonstrated that CT derived radiomics features were affected by temporary oxygen changes and hypoxia targeted treatment. The identified features correlated with hypoxia PET tracer HX4. Therefore, radiomics might offer a promising non-radioactive imaging approach for detecting hypoxia

\section{Acknowledgements}

The authors acknowledge the support of the QulC-ConCePT project, partly funded by EFPIA companies and the Innovative Medicine Initiative Joint Undertaking (IMI JU) under Grant Agreement No. 115151. 


\section{Figures:}

Figure 1: Graphical representation of feature selection procedure

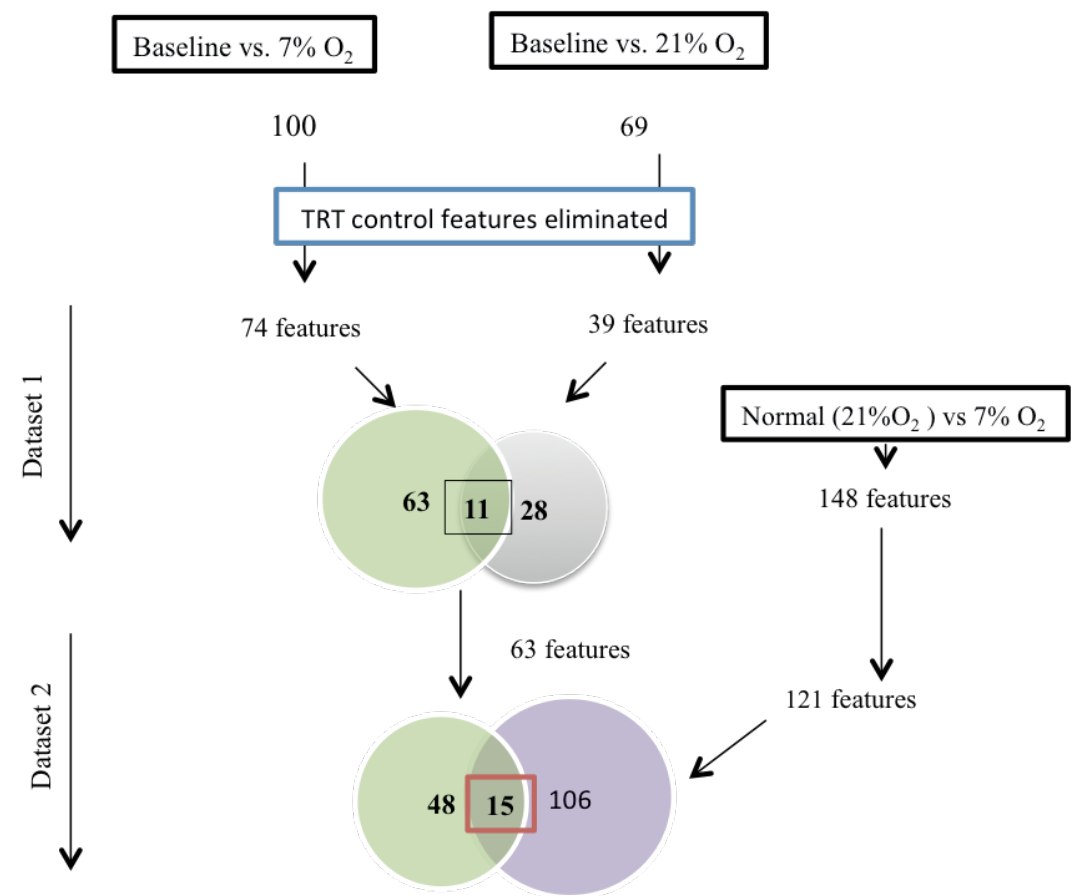


Figure 2: Radiomics features related to oxygen change (7\%)

A) Correlation of features with SUVmean and B) difference in feature between $21 \%$ and $7 \%$ $\mathrm{O}_{2}$ breathing conditions for features LoG sigma $10 \mathrm{~mm} 3 \mathrm{D}$ first order statistics entropy and LoG sigma $10 \mathrm{~mm}$ 3D first order statistics uniformity.

A

LoG sigma 10mm 3D first order statistics entropy

Dataset 1

Dataset 2
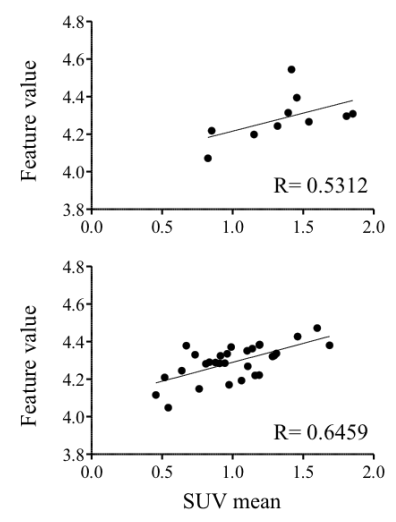

B

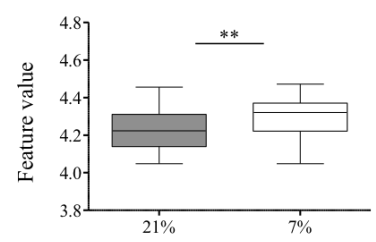

LoG sigma 10mm 3D first order statistics uniformity
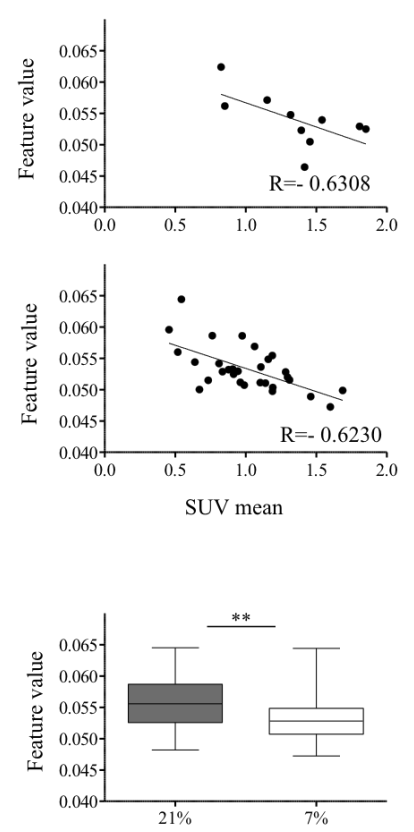
Figure 3: Radiomics features related to hypoxia targeting

A) Graphical representation of the feature selection procedure. B) Correlation of feature (run low gray level emphasis) with SUVmean and tumor to background ratios (TBR) in preclinical and clinical settings

A LoG sigma $10 \mathrm{~mm} 3 \mathrm{D}$ first order statistics entropy

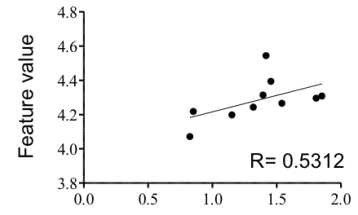

Dataset 2

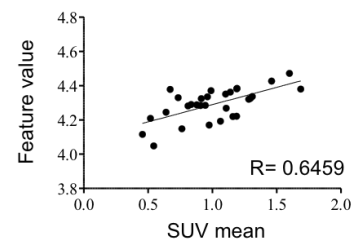

B

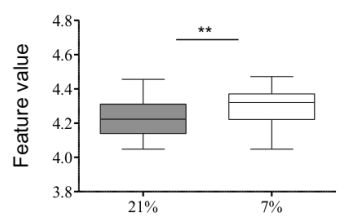

LoG sigma $10 \mathrm{~mm} 3 \mathrm{D}$ first order statistics uniformity
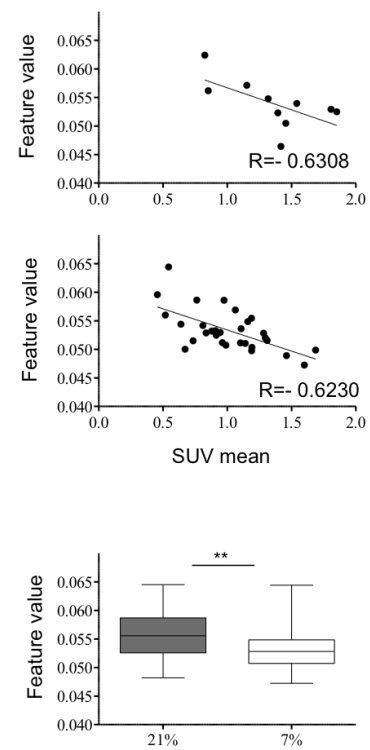
Figure 4: Correlation of radiomics feature (wavelet LLL gray level size zone variability) to A) hypoxic fraction and B) hypoxic volume

Wavelet LLL gray level size zone variability

A

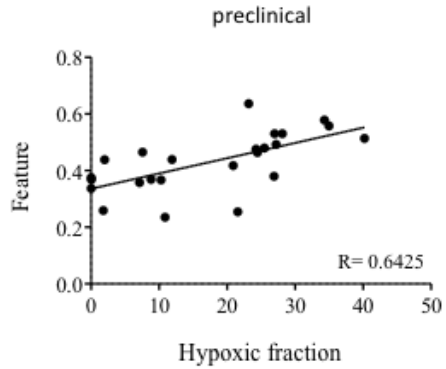

B

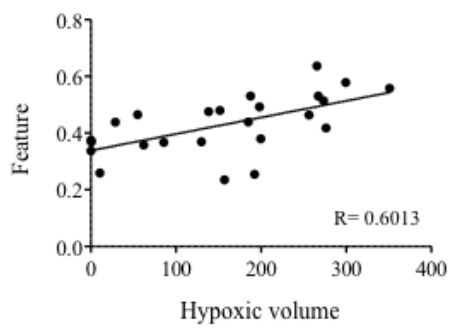

clinical
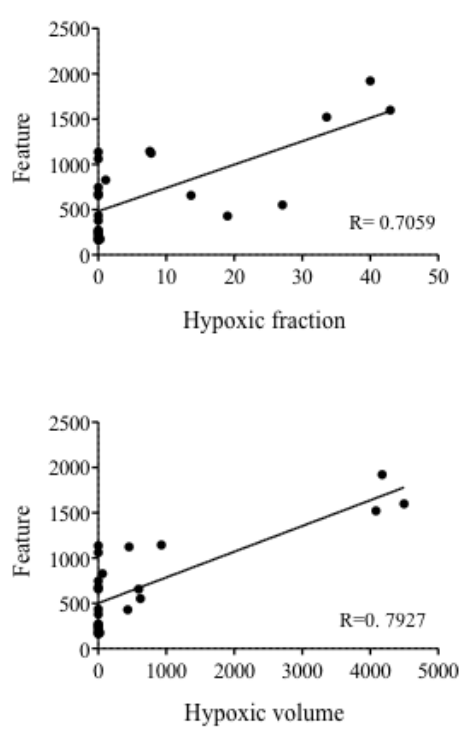


\section{Supplementary figures}

Supplementary figure 1: Schematic representation of [18F]-HX4 PET/CT scanning and oxygen modification in dataset 1 and dataset 2 . The black arrows show time in hours and blue arrows shows the duration of oxygen modification. The colored boxes represent [18F]HX4 PET/CT scan acquisition. The comparison of features is made between time points represented in same colored boxes.

Dataset 1

control

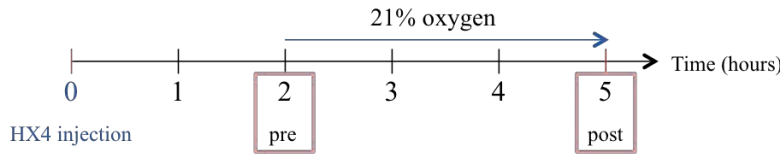

Dataset 2
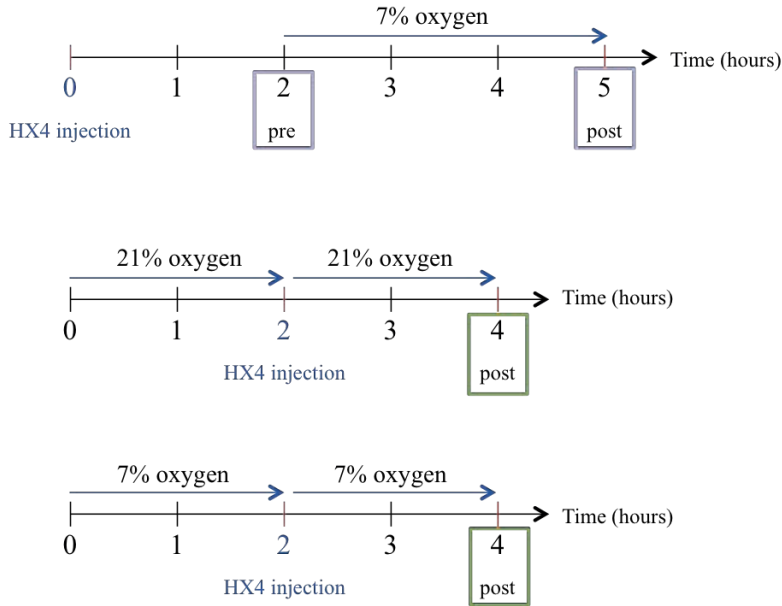
Supplementary figure 2: A) change in radiomic feature (wavelet LLL gray level size zone variability) due to $\mathrm{TH} 302$ treatment and $\mathrm{B}$ ) correlation to tumor volume

Wavelet LLL gray level size zone variability

A

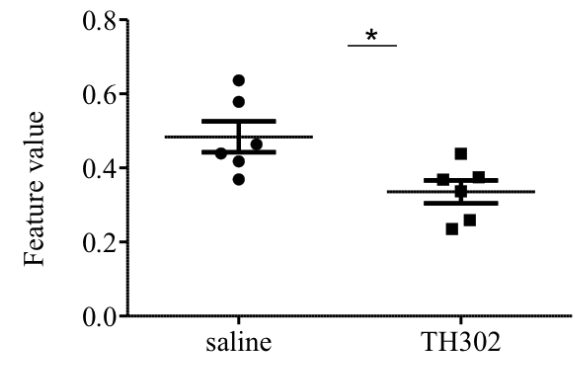

B

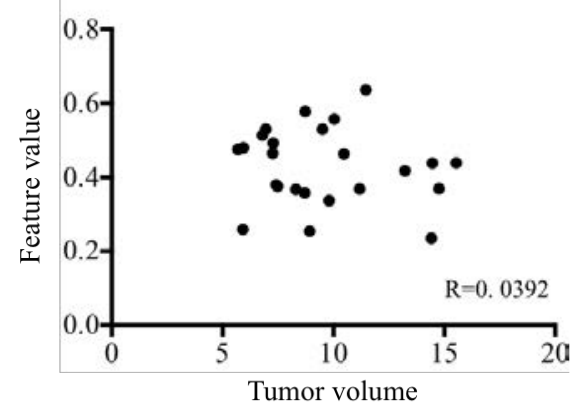


Supplementary table 1: Correlation of features related to $7 \% \mathrm{O}_{2}$ breathing in rat rhabdomyosarcoma tumors with $\left[{ }^{18} \mathrm{~F}\right] \mathrm{HX} 4$ SUV mean and TBR. $\left({ }^{*}\right.$ indicates significant where $\left.{ }^{*} \mathrm{P}<0.05, * * \mathrm{P}<0.01,{ }^{*} * \mathrm{P}<0.001\right)$. Moderate $(\mathrm{R}>0.5)$ and high $(\mathrm{R}>0.6)$ correlations are highlighted in gray

\begin{tabular}{|c|c|c|c|c|c|}
\hline \multirow[t]{2}{*}{ Feature } & \multicolumn{2}{|c|}{ Training dataset } & \multicolumn{2}{|c|}{ Validation dataset } & \multirow{2}{*}{$\begin{array}{c}\text { Change in } \\
\text { feature with } \\
7 \% \text { breathing } \\
\end{array}$} \\
\hline & $\begin{array}{c}7 \% \text { SUV } \\
\text { mean }\end{array}$ & $\begin{array}{c}7 \% \text { TBR } \\
\text { mean } \\
\end{array}$ & $\begin{array}{l}7 \% \text { SUV } \\
\text { mean }\end{array}$ & $\begin{array}{c}7 \% \text { TBR } \\
\text { mean }\end{array}$ & \\
\hline LoG 10mm 3D entropy & $\begin{array}{c}R= \\
0.5312\end{array}$ & $\begin{array}{c}* R= \\
0.8342\end{array}$ & $\begin{array}{l}* * * \mathrm{R}= \\
0.6459\end{array}$ & $\begin{array}{c}R= \\
0.3703\end{array}$ & $* *$ increase \\
\hline $\begin{array}{l}\text { LoG } 10 \mathrm{~mm} 3 \mathrm{D} \\
\text { entropyPos }\end{array}$ & $\begin{array}{c}R= \\
0.5312\end{array}$ & $\begin{array}{c}* R= \\
0.8342\end{array}$ & $\begin{array}{l}* * \mathrm{R}= \\
0.6459\end{array}$ & $\begin{array}{c}R= \\
0.3703\end{array}$ & $* *$ increase \\
\hline LoG $10 \mathrm{~mm} 3 \mathrm{D}$ mean & $\begin{array}{c}R= \\
0.4426\end{array}$ & $\begin{array}{c}R= \\
0.4474\end{array}$ & $\begin{array}{l}* * * \mathrm{R}= \\
0.6527\end{array}$ & $\begin{array}{c}* R= \\
0.4013\end{array}$ & $* * *$ increase \\
\hline LoG $10 \mathrm{~mm}$ 3D meanPos & $\begin{array}{c}R= \\
0.4426\end{array}$ & $\begin{array}{c}R= \\
0.4474\end{array}$ & $\begin{array}{l}* * * \mathrm{R}= \\
0.6527\end{array}$ & $\begin{array}{c}* R= \\
0.4013\end{array}$ & $* * *$ increase \\
\hline LoG $10 \mathrm{~mm}$ 3D uniformity & $\begin{array}{c}R=- \\
0.6308\end{array}$ & $\begin{array}{c}* R=- \\
0.8037\end{array}$ & $\begin{array}{l}* * \mathrm{R}=- \\
0.6230\end{array}$ & $\begin{array}{c}R=- \\
0.3733\end{array}$ & ** decrease \\
\hline $\begin{array}{l}\text { LoG } 10 \mathrm{~mm} 3 \mathrm{D} \\
\text { uniformityPos }\end{array}$ & $\begin{array}{c}R=- \\
0.6308\end{array}$ & $\begin{array}{c}* R=- \\
0.8037\end{array}$ & $\begin{array}{l}* * \mathrm{R}=- \\
0.6230\end{array}$ & $\begin{array}{c}R=- \\
0.3733\end{array}$ & ** decrease \\
\hline $\begin{array}{l}\text { LoG } 4 \mathrm{~mm} \text { 3D standard } \\
\text { deviation }\end{array}$ & $\begin{array}{c}R= \\
0.4282\end{array}$ & $\begin{array}{c}R= \\
0.5833\end{array}$ & $\begin{array}{c}* R= \\
0.3894\end{array}$ & $\begin{array}{c}R= \\
0.1443\end{array}$ & $* *$ increase \\
\hline LoG 9mm 3D mean & $\begin{array}{c}R= \\
0.2832\end{array}$ & $\begin{array}{c}R= \\
0.1479\end{array}$ & $\begin{array}{l}* * \mathrm{R}= \\
0.4897\end{array}$ & $\begin{array}{c}* \mathrm{R}= \\
0.2766\end{array}$ & $* *$ increase \\
\hline LoG 9mm 3D meanPos & $\begin{array}{c}R= \\
0.2832\end{array}$ & $\begin{array}{c}R= \\
0.1479\end{array}$ & $\begin{array}{c}* R= \\
0.4046\end{array}$ & $\begin{array}{c}* R= \\
0.2766\end{array}$ & $* *$ increase \\
\hline LoG 9.5 mm 3D mean & $\begin{array}{c}R= \\
0.3854\end{array}$ & $\begin{array}{c}R= \\
0.3362\end{array}$ & $\begin{array}{l}* * * \mathrm{R}= \\
0.5972\end{array}$ & $\begin{array}{c}* \mathrm{R}= \\
0.3578\end{array}$ & $* * *$ increase \\
\hline LoG $9.5 \mathrm{~mm}$ 3D meanPos & $\begin{array}{c}R= \\
0.3854\end{array}$ & $\begin{array}{c}R= \\
0.3362\end{array}$ & $\begin{array}{l}* * * \mathrm{R}= \\
0.5972\end{array}$ & $\begin{array}{c}* R= \\
0.3578\end{array}$ & $* * *$ increase \\
\hline Shape compactness & $\begin{array}{c}\mathrm{R}= \\
0.3376\end{array}$ & $\begin{array}{c}* \mathrm{R}= \\
0.8183\end{array}$ & $\begin{array}{l}* * * \mathrm{R}= \\
0.6717\end{array}$ & $\begin{array}{l}* * \mathrm{R}= \\
0.5854\end{array}$ & $*$ increase \\
\hline Shape maxDiameter2Dz & $\begin{array}{c}R= \\
0.3978\end{array}$ & $\begin{array}{c}* R= \\
0.7768\end{array}$ & $\begin{array}{l}* * * \mathrm{R}= \\
0.7204\end{array}$ & $\begin{array}{c}* * \mathrm{R}= \\
0.5415\end{array}$ & $*$ increase \\
\hline $\begin{array}{l}\text { Wavelet LHH gray level } \\
\text { co-occurrence matrix inv } \\
\text { Diffnorm }\end{array}$ & $\begin{array}{c}R= \\
0.3349\end{array}$ & $\begin{array}{c}R= \\
0.4158\end{array}$ & $\begin{array}{c}R= \\
0.1543\end{array}$ & $\begin{array}{c}R=- \\
0.0023\end{array}$ & $*$ increase \\
\hline $\begin{array}{l}\text { Wavelet LHL first order } \\
\text { statistics mean }\end{array}$ & $\begin{array}{c}R= \\
0.0623\end{array}$ & $\begin{array}{c}* R= \\
0.7386\end{array}$ & $\begin{array}{c}* R= \\
0.4249\end{array}$ & $\begin{array}{c}R= \\
0.2729\end{array}$ & * increase \\
\hline
\end{tabular}


Supplementary Table 2: Correlation of significant features with HX4 uptake (SUV mean and TBR), Hypoxic fraction (HF) and hypoxic volume (HV) in rat rhabdomyosarcoma and human lung cancer ( ${ }^{*} \mathrm{P}<0.05,{ }^{* *} \mathrm{P}<0.01,{ }^{* * *} \mathrm{P}<0.001,{ }^{* * * * \mathrm{P}<0.0001)}$

\begin{tabular}{|c|c|c|c|c|c|c|c|c|}
\hline & \multicolumn{4}{|c|}{ Preclinical Dataset } & \multicolumn{4}{|c|}{ Clinical dataset } \\
\hline Feature & $\begin{array}{l}\text { SUV } \\
\text { mean }\end{array}$ & TBR & HF & HV & $\begin{array}{l}\text { SUV } \\
\text { mean }\end{array}$ & TBR & $\mathrm{HF}$ & HV \\
\hline $\begin{array}{l}\text { Gray level } \\
\text { size zone } \\
\text { matrix } \\
\text { high } \\
\text { intensity } \\
\text { emphasis }\end{array}$ & $\begin{array}{c}\mathrm{R}= \\
0.4632 \\
\\
*\end{array}$ & $\begin{array}{c}R= \\
0.2467\end{array}$ & $\begin{array}{c}R= \\
0.2288\end{array}$ & $\begin{array}{c}R= \\
0.3341\end{array}$ & $\begin{array}{c}\mathrm{R}= \\
0.2028\end{array}$ & $\begin{array}{c}\mathrm{R}= \\
0.4943 \\
* *\end{array}$ & $\begin{array}{c}R= \\
0.0987\end{array}$ & $\begin{array}{c}R= \\
0.0446\end{array}$ \\
\hline $\begin{array}{l}\text { Gray level } \\
\text { size zone } \\
\text { matrix } \\
\text { high } \\
\text { intensity } \\
\text { small area } \\
\text { Emphasis }\end{array}$ & $\begin{array}{c}R= \\
0.4082\end{array}$ & $\begin{array}{c}R= \\
0.2467\end{array}$ & $\begin{array}{c}R= \\
0.2477\end{array}$ & $\begin{array}{c}R= \\
0.2863\end{array}$ & $\begin{array}{c}R= \\
0.1668\end{array}$ & $\begin{array}{c}R= \\
0.4836\end{array}$ & $\begin{array}{c}R= \\
0.1215\end{array}$ & $\begin{array}{c}R= \\
0.0445\end{array}$ \\
\hline $\begin{array}{l}\text { Long run } \\
\text { low gray } \\
\text { Level } \\
\text { emphasis }\end{array}$ & $\begin{array}{c}\mathrm{R}=- \\
0.4811 \\
*\end{array}$ & $\begin{array}{c}\mathrm{R}= \\
0.5277 \\
* *\end{array}$ & $\begin{array}{c}\mathrm{R}=- \\
0.4562 \\
*\end{array}$ & $\begin{array}{c}\mathrm{R}=- \\
0.4781 \\
*\end{array}$ & $\begin{array}{c}R=- \\
0.3386\end{array}$ & $\begin{array}{c}\mathrm{R}=- \\
0.5421 \\
* *\end{array}$ & $\begin{array}{c}R=- \\
0.0511\end{array}$ & $\begin{array}{c}R=- \\
0.1583\end{array}$ \\
\hline $\begin{array}{l}\text { Low gray } \\
\text { level run } \\
\text { emphasis }\end{array}$ & $\begin{array}{c}\mathrm{R}=- \\
0.5146 \\
* *\end{array}$ & $\begin{array}{c}\mathrm{R}= \\
0.5444 \\
* *\end{array}$ & $\begin{array}{c}\mathrm{R}=- \\
0.4602 \\
*\end{array}$ & $\begin{array}{c}\mathrm{R}=- \\
0.5065 \\
*\end{array}$ & $\begin{array}{c}\mathrm{R}=- \\
0.4240 \\
*\end{array}$ & $\begin{array}{c}\mathrm{R}=- \\
0.5756 \\
* * *\end{array}$ & $\begin{array}{c}R=- \\
0.1344\end{array}$ & $\begin{array}{c}R=- \\
0.1583\end{array}$ \\
\hline $\begin{array}{c}\text { Short run } \\
\text { low gray } \\
\text { lev } \\
\text { emphasis }\end{array}$ & $\begin{array}{c}\mathrm{R}=- \\
0.5186 \\
* *\end{array}$ & $\begin{array}{c}\mathrm{R}= \\
0.5315 \\
* *\end{array}$ & $\begin{array}{c}\mathrm{R}=- \\
0.4550 \\
*\end{array}$ & $\begin{array}{c}\mathrm{R}=- \\
0.5067 \\
*\end{array}$ & $\begin{array}{c}\mathrm{R}=- \\
0.4381 \\
*\end{array}$ & $\begin{array}{c}\mathrm{R}=- \\
0.5850 \\
* * *\end{array}$ & $\begin{array}{c}R=- \\
0.1552\end{array}$ & $\begin{array}{c}R=- \\
0.1752\end{array}$ \\
\hline $\begin{array}{l}\text { Wavelet } \\
\text { LLL gray } \\
\text { level size } \\
\text { zone } \\
\text { matrix } \\
\text { variability }\end{array}$ & $\begin{array}{c}R= \\
0.5501\end{array}$ & $\begin{array}{c}R= \\
0.6146\end{array}$ & $\begin{array}{c}R= \\
0.6425\end{array}$ & $\begin{array}{c}R= \\
0.6013\end{array}$ & $\begin{array}{c}R= \\
0.0196\end{array}$ & $\begin{array}{c}R= \\
0.5364\end{array}$ & $\begin{array}{c}R= \\
0.7059\end{array}$ & $\begin{array}{c}R= \\
0.7927\end{array}$ \\
\hline
\end{tabular}




\section{References}

1 Dubois LJ, Niemans R, van Kuijk SJA, et al. New ways to image and target tumour hypoxia and its molecular responses. Radiotherapy and Oncology 2015;116:352357.

2 Höckel M Vaupel P. Tumor Hypoxia: Definitions and Current Clinical, Biologic, and Molecular Aspects. Journal of the National Cancer Institute 2001;93:266-276.

3 Brown JM Wilson WR. Exploiting tumour hypoxia in cancer treatment. Nat Rev Cancer 2004;4:437-447.

4 Krohn KA, Link JM Mason RP. Molecular Imaging of Hypoxia. Journal of Nuclear Medicine 2008;49:129S-148S.

5 van Loon J, Janssen MM, Öllers M, et al. PET imaging of hypoxia using [18F]HX4: a phase I trial. European Journal of Nuclear Medicine and Molecular Imaging 2010;37:1663-1668.

6 Dubois LJ, Lieuwes NG, Janssen MHM, et al. Preclinical evaluation and validation of [18F]HX4, a promising hypoxia marker for PET imaging. Proceedings of the National Academy of Sciences 2011;108:14620-14625.

7 Zegers CML, van Elmpt W, Wierts R, et al. Hypoxia imaging with [18F]HX4 PET in NSCLC patients: Defining optimal imaging parameters. Radiotherapy and Oncology 2013;109:58-64.

8 Zegers CML, van Elmpt W, Reymen B, et al. In Vivo Quantification of Hypoxic and Metabolic Status of NSCLC Tumors Using [18F]HX4 and [18F]FDG-PET/CT Imaging. Clinical Cancer Research 2014;20:6389-6397.

9 Peeters SGJA, Zegers CML, Lieuwes NG, et al. A Comparative Study of the Hypoxia PET Tracers [18F]HX4, [18F]FAZA, and [18F]FMISO in a Preclinical Tumor Model. International Journal of Radiation Oncology*Biology*Physics 2015;91:351-359.

10 Zegers CL, van Elmpt W, Szardenings K, et al. Repeatability of hypoxia PET imaging using [18F]HX4 in lung and head and neck cancer patients: a prospective multicenter trial. European Journal of Nuclear Medicine and Molecular Imaging 2015;42:1840-1849.

11 Wack LJ, Mönnich D, van Elmpt W, et al. Comparison of [18F]-FMISO, [18F]-FAZA and [18F]-HX4 for PET imaging of hypoxia - a simulation study. Acta Oncologica 2015;54:1370-1377.

12 Zegers CML, van Elmpt W, Hoebers FJP, et al. Imaging of tumour hypoxia and metabolism in patients with head and neck squamous cell carcinoma. Acta Oncologica 2015;54:1378-1384.

13 Peeters SGJA, Zegers CML, Biemans R, et al. TH-302 in Combination with Radiotherapy Enhances the Therapeutic Outcome and Is Associated with Pretreatment [18F]HX4 Hypoxia PET Imaging. Clinical Cancer Research 2015;21:2984-2992.

14 Lambin P, Rios-Velazquez E, Leijenaar R, et al. Radiomics: Extracting more information from medical images using advanced feature analysis. European Journal of Cancer 2012;48:441-446.

15 Kumar V, Gu Y, Basu S, et al. Radiomics: the process and the challenges. Magnetic Resonance Imaging 2012;30:1234-1248.

16 Aerts HJWL, Velazquez ER, Leijenaar RTH, et al. Decoding tumour phenotype by noninvasive imaging using a quantitative radiomics approach. Nat Commun 2014;5.

17 Parmar C, Rios Velazquez E, Leijenaar R, et al. Robust Radiomics Feature Quantification Using Semiautomatic Volumetric Segmentation. PLoS ONE 2014;9:e102107.

18 Leijenaar RTH, Carvalho S, Velazquez ER, et al. Stability of FDG-PET Radiomics 
features: An integrated analysis of test-retest and inter-observer variability. Acta Oncologica 2013;52:1391-1397.

19 Velazquez ER, Parmar C, Jermoumi M, et al. Volumetric CT-based segmentation of NSCLC using 3D-Slicer. Scientific Reports 2013;3:3529.

20 Coroller TP, Grossmann P, Hou Y, et al. CT-based radiomic signature predicts distant metastasis in lung adenocarcinoma. Radiotherapy and Oncology 2015;114:345-350.

21 Leijenaar RTH, Nalbantov G, Carvalho S, et al. The effect of SUV discretization in quantitative FDG-PET Radiomics: the need for standardized methodology in tumor texture analysis. Scientific Reports 2015;5:11075.

22 Panth KM, Leijenaar RTH, Carvalho S, et al. Is there a causal relationship between genetic changes and radiomics-based image features? An in vivo preclinical experiment with doxycycline inducible GADD34 tumor cells. Radiotherapy and Oncology 2015;116:462-466.

23 Parmar C, Leijenaar RTH, Grossmann P, et al. Radiomic feature clusters and Prognostic Signatures specific for Lung and Head \&amp; Neck cancer. Scientific Reports 2015;5:11044.

24 Parmar C, Grossmann P, Bussink J, Lambin P Aerts HJWL. Machine Learning methods for Quantitative Radiomic Biomarkers. Scientific Reports 2015;5:13087.

25 Carvalho S, Leijenaar RTH, Velazquez ER, et al. Prognostic value of metabolic metrics extracted from baseline positron emission tomography images in nonsmall cell lung cancer. Acta Oncologica 2013;52:1398-1404.

26 Shannon AM, Bouchier-Hayes DJ, Condron CM Toomey D. Tumour hypoxia, chemotherapeutic resistance and hypoxia-related therapies. Cancer Treatment Reviews 2003;29:297-307.

27 Yaromina A, Kroeber T, Meinzer A, et al. Exploratory Study of the Prognostic Value of Microenvironmental Parameters During Fractionated Irradiation in Human Squamous Cell Carcinoma Xenografts. International Journal of Radiation Oncology*Biology*Physics 2011;80:1205-1213.

28 Dubois L, Landuyt W, Haustermans K, et al. Evaluation of hypoxia in an experimental rat tumour model by [(18)F]Fluoromisonidazole PET and immunohistochemistry. British Journal of Cancer 2004;91:1947-1954. 
Chapter 7

\section{Discussion and future perspectives}




\section{General discussion and summary}

Non-invasive imaging reflects the tumor heterogeneity in a comprehensive manner both spatially and longitudinally and plays a central role in the context of personalized medicine and aids in cancer diagnosis, staging, treatment planning and treatment follow-up ${ }^{1-3}$. In the last decade, innovations have been made mainly in four categories: 1 ) development of novel imaging biomarkers (tracers, probes or antibodies), 2) improvement in imaging equipment (hardware), 3) standardization of imaging protocols for multicentric use and 4) advancements in image analysis $^{4}$. In this thesis, we focused on two of these categories by evaluating novel imaging biomarkers (EGFR and MMP2 antibody imaging) and advanced image analysis (radiomics).

An ideal biomarker not only provides information on a biological process in normal and pathological condition but also provides knowledge on drug targets and the treatment responses which are associated with the selected clinical endpoint ${ }^{5,6}$. Therefore, an imaging biomarker should be employed to predict responses from a treatment directed to a specific target. In other words, the imaging biomarker and the drug should ideally have the same target - a protein of interest (e.g. EGFR, MMP2) or a biological process (hypoxia) - to be able to detect treatment responses which provide information not only on drug efficacy but also about the drug reachability in the tumor. Such approach can open the possibility for new clinical trial designs such as 'window-of-opportunity' trial, which is discussed in chapter 2 . This window will aid in selecting new drugs at an early stage that will potentially benefit the patient. In a preclinical experiment, our group has used this concept to show that ${ }^{18} \mathrm{~F}-\mathrm{HX} 4$ PET hypoxia imaging can be used to monitor treatment efficacy of the hypoxia-activated prodrug $\mathrm{TH} 302$. Additionally, this study indicated that the pretreatment ${ }^{18} \mathrm{~F}-\mathrm{HX} 4$ PET assessed hypoxic fraction was predictive for the TH302 treatment responses ${ }^{7}$. One could think of using ${ }^{18}$ F-FDG as agent to assess the metabolic response after therapy. However, in this scenario, it would not be ideal to use ${ }^{18}$ F-FDG as it was clearly reported that ${ }^{18} \mathrm{~F}$-FDG positive metabolic volumes and ${ }^{18} \mathrm{~F}-\mathrm{HX} 4$ positive hypoxic volumes have distinct uptake patterns ${ }^{8}$. Thus, employing the relevant imaging biomarker will enable progression in effective drug-development and also in individualizing patient treatment, which forms the basis for the first part of this thesis. In chapter 3 and 4 we evaluated antibodies targeting EGFR and MMP2 respectively as new antibody-based molecular imaging probes. 
Antibody-based imaging is attractive because of the high specificity of the antibody towards the target ${ }^{9}$. van Dongen et al described antibody-based imaging as performing 'comprehensive immunohistochemistry in vivo' ${ }^{10}$. Imaging can be performed using FDA approved antibodies or using newly developed antibodies. Using FDA approved antibodies the process of qualification for clinical use is faster as the pharmacokinetics and efficacy of the antibody are pre-determined ${ }^{10,11}$. However, the labeling of such antibodies should be done in such a manner that it doesn't affect the properties of the antibody. In chapter 3, we used cetuximab, an FDA approved monoclonal antibody ( $\mathrm{mAb}$ ) for targeting EGFR. For immunoPET studies (antibody-based imaging using PET), the antibody labeling is usually done with radioisotopes with relatively longer half-lives as intact antibodies have longer circulating time in the body and optimal time point ranges from 2 to 4 days for achieving optimal imaging conditions reflected in high tumor to background ratios. Commonly used radioisotopes for immuno-PET are ${ }^{64} \mathrm{Cu}\left(\mathrm{t}_{1 / 2}=12.7\right.$ hours $),{ }^{86} \mathrm{Y}\left(\mathrm{t}_{1 / 2}=14.7\right.$ hours), ${ }^{124} \mathrm{I}$ ( $\mathrm{t}_{1 / 2}=100.3$ hours), ${ }^{89} \mathrm{Zr}$ ( $\mathrm{t}_{1 / 2}=78.4$ hours). ${ }^{124} \mathrm{I}$ and ${ }^{89} \mathrm{Zr}$ are more suitable for imaging intact antibodies as their half-life matches the optimal time point for imaging ${ }^{10} .{ }^{89} \mathrm{Zr}$ has many advantages over ${ }^{124}$ I such as low cost of production, high in vivo stability and relatively low energy positron which enables high spatial resolution ${ }^{12-14} .{ }^{124}$ I is tagged to mAbs via tyrosine residues, which undergoes dehalogenation in vivo leading to unspecific uptake in other organs, such as the thyroid. Besides, when mAbs are internalized after binding to receptor, rapid elimination of ${ }^{121}$, occurs inside the cell due to lysis and therefore do not represent actual uptake in the tumor whereas ${ }^{89} \mathrm{Zr}$ accumulates in the tumor ${ }^{15-17}$. With recent advances in antibody engineering, antibody fragments for targeting and imaging are being developed ${ }^{10,18}$. The advantage of smaller antibody fragments over intact antibodies is that the clearance from blood is faster with retaining high specificity towards the target. Some commonly used formats are antigen binding fragment (Fab), single chain variable fragment (ScFv), diabodies (ScFv dimers) and third generation antibodies also named miniature antibodies or small immunoantibodies $^{19,20}$. The small immuno-antibody format is used for imaging MMP2 which is described in chapter 4 . The main advantage of small immune-antibodies is that these molecules are more stable compared to ScFv and Fab fragments and thus the reachability to the tumor is higher ${ }^{21}$. On the other hand, the specificity of the antibody is comparable to the full antibody. For such antibodies, radioisotopes with shorter half-life such as ${ }^{18} \mathrm{~F}$ or ${ }^{64} \mathrm{Cu}$ can also be used as the accumulation in tumor and clearance from blood is faster. Although synthesis of ${ }^{18} \mathrm{~F}$ labeled probes is more time-consuming compared to ${ }^{64} \mathrm{Cu}$, these ${ }^{18} \mathrm{~F}$ probes are more 
stable in vivo than ${ }^{64} \mathrm{Cu}^{22}$.

EGFR is overexpressed in many cancers and its level of expression is often linked to tumor aggressiveness and worse prognosis (REF). Cetuximab (Erbitux) is a monoclonal antibody mainly used for treatment of colorectal and head and neck cancers ${ }^{23}$. However, not all patients treated with cetuximab do benefit from this treatment ${ }^{24}$. Furthermore, EGFR expression levels prior to therapy has been a poor predictor for selecting patients that benefit from cetuximab treatment ${ }^{24,25}$. A clear disparity between EGFR expression and cetuximab uptake has also been demonstrated by immuno-PET. One of the main reasons for this disparity might be the fact that antibody uptake can be influenced by tumor microenvironmental characteristics ${ }^{26-28}$. In chapter 3 , we determined in three patient derived tumor models varying in EGFR expression if and which tumor microenvironmental characteristics influenced cetuximab uptake. Patient derived xenograft (PDX) models retain and represent the tumor characteristics of the parent tumor to a larger extent compared to cell line derived tumors ${ }^{29,30}$. Also in these PDX models, a clear disparity was observed between EGFR expression and cetuximab uptake. We found that the overall uptake in all three tumors was correlated to the relative microvessel density and the hypoxic fraction (HF). Angiogenesis stimulating factors such as VEGF are highly induced under hypoxic conditions $^{31-34}$ and therefore, correlation to both hypoxia and microvessel density can be expected. When the correlation was restricted to each tumor model separately, for two out of three models a high correlation was observed between tumor to blood ratio (TBR) and microvessel density indicating that microvessel density is the direct confounding factor for the uptake of the antibody. It was reported that the delivery of drugs and macromolecules largely depends on the microvessel density $^{26,35}$. One alternative could be the use of small antibody fragments that have larger reachability to the tumor. Studies have shown that the uptake of EGFR targeting small antibodies or nanobodies correlated better to EGFR expression indicating maximum reachability of these antibodies to the tumor ${ }^{36,37}$. Thus, smaller sized antibody fragments could provide a promising alternative for effective targeting of the tumor.

Early detection of tumor invasion can potentially aid in preventing metastasis $^{38,39}$. However, no biomarker is yet clinically available for imaging invasion. MMP2 expression has been directly linked to invasion and metastasis ${ }^{40-42}$. It was reported that inhibiting MMP2 has led to reduced invasion and metastases ${ }^{43-45}$. Hence, imaging MMP2 could serve as a biomarker for invasion. In chapter 4 , we evaluated the uptake of the MMP2 targeting small immuno protein (SIP) antibody in tumors with 
varying MMP2 expression. For this application, the MMP2 targeting SIP antibody was tagged to Cy5 for enabling near infrared fluorescence imaging (NIRF). MMP2 imaging has been performed previously using probes that can be activated by MMP2 activity. However, the main disadvantage of such probes is that the peptide targeting MMP2 is linked to polyethylene glycol (PEG) or lipids which make the nature of the probe bulky. Moreover, the cleavage can also occur due to activity of other gelatinases in the body ${ }^{46}$. Therefore, an antibody specific to MMP2 was designed in SIP format (aMMP2-SIP) for clinical translatability and also for overcoming the challenges faced with intact antibodies. The antibody specificity was determined by comparing the uptake in MMP2 expressing tumors with their corresponding MMP2 knockdown models. We have observed that 24 hour post injection of the antibody, the tumor to background ratios showed clear differences between high and low MMP2 expressing tumors in U87. Additionally, we have found that the uptake of the antibody was dependent on MMP2 activity rather than on MMP2 expression. In two invasive models, although MMP2 expression was relatively low in knockdown models compared to the control tumors, the activity was unchanged. This indicates that probably even low MMP2 expression might be enough for maintaining MMP2 activity.

In the second part of the thesis, we focused on evaluating the link between tumor heterogeneities and radiomics features. Radiomics hypothesizes that intra-tumoral heterogeneities are hidden in medical images and can be captured by imaging features ${ }^{4,47-49}$. Radiomics signatures were shown to be prognostic in human cancers and were associated with gene signatures ${ }^{50-52}$. However, the direct link between underlying genetic factors and radiomics features is not fully established. Therefore, in a preclinical model, we have assessed the causal relationship between radiomics features and a known genetic change. The main advantage of using preclinical studies is that 'known' consequences of a genetic change or a therapeutic intervention can be directly evaluated. Moreover, proper controls can be used to ascertain selection of the right features, which is impractical in clinical settings. In chapter 5, we evaluated if a genetic change induced by doxycycline administration (GADD34 overexpression) could directly influence radiomics. Consistent features were found when the genetic change was combined with radiation treatment at early and late time points after the induction of the genetic change, which could not be detected by naked eye visual inspection. Mainly features derived from first order statistics or textural features were affected, indicating changes in intra-tumoral heterogeneities. However, no features were affected for the 
experimental group with only gene induction similar to the tumor growth data where only gene induction did not affect the tumor growth. Moreover, the scanning parameters and quality of images can directly influence the imaging features ${ }^{46,53}$. The high resolution micro-CT images showed artifacts due to beam hardening and scatter from bone which could have affected the image features to some extent. However, most of the images showed these artifacts and therefore randomness occurring due to artifacts was eliminated by test-retest analysis. We also determined if a different energy could affect the features as the attenuation of $x$-rays not only depends on tissue but also on energy. For this, we compared the features obtained from 40 and $80 \mathrm{kVp}$ energy CT images. Similar results were observed for both energies indicating the reliability of the features despite of different energy sources used. In future, the association of radiomics features to the molecular information derived from the dual energy CT images (DECT) will be determined.

The direct link between the imaging features and intra-tumoral heterogeneities is not fully established. Hypoxia, a tumor microenvironmental characteristic causes treatment resistance and poor prognosis $^{54-57}$ and is usually evaluated with PET imaging. However, the availability of PET depends on factors such as time, cost and facilities for tracer production etc. Therefore, we evaluated if modulating tumor oxygenation can influence the CT derived imaging features (chapter 6). Temporary changes in oxygen due to low oxygen breathing (7\%) led to significant changes in features mainly derived from first order statistics indicating changes in deviation of discrete intensity levels. Next, when treated with drugs targeting hypoxia, features mainly related to texture demonstrated significant changes in both preclinical and clinical settings. These textural features indicate tumor heterogeneities at a regional level. However, no overlapping features were found between the features related to temporary changes in oxygen (7\% oxygen breathing) and features due to prolonged hypoxia-targeted treatment. Clearly, temporary and prolonged changes in hypoxia had a different impact on the imaging features. While, the first order intensity features due to $7 \%$ oxygen breathing indicate changes in gray level intensities of the images, they do not provide information on the spatial distribution of gray level patterns, which were only changed upon hypoxia reduction with $\mathrm{TH} 302$ or nitroglycerin. The main advantage of this proof-of-concept study compared to chapter 5 is that both preclinical and clinical images were acquired on the same clinical scanner and therefore, the deviations caused by scanner itself are low. This proof-of-concept study shows that quantifying tumor heterogeneities such as hypoxia is possible by 
radiomics approach. However, radiomics features as such cannot provide spatial hypoxia information, which can be visualized on PET images. One of the major limitations of the preclinical studies described in this thesis is that overlapping features between groups were not corrected for multiple testing due to smaller group sizes. Due to large number of features, the chance of finding significantly different features between groups also increase. However, selecting features based on test-retest methods and also using adequate controls in each dataset allows for proper selection of features. Furthermore, similar behavior of features irrespective of time and dataset used explains that these features are reliable. We have observed that features that were found in both preclinical and clinical datasets upon treatment aimed to reduce hypoxic fraction showed similar correlations with ${ }^{18} \mathrm{~F}-\mathrm{HX} 4$ in both datasets (Chapter 6). Nevertheless, once the features are characterized for the underlying genetic factors and tumor heterogeneities using preclinical datasets, validation in clinical datasets is warranted. Relevant models are necessary for validation since cancer origin and complex tumor characteristics in patients might be a bottleneck for translation. 


\section{Conclusions and future perspectives}

Non-invasive imaging has become an integral part of personalized medicine in cancer. In this thesis, we evaluated novel molecular imaging methods for cancer detection including the evaluation of new imaging tracers and advanced image analysis methodologies. Proper imaging tracers are required for selecting patients that may respond to a treatment and to avoid unnecessary treatment associated with increased costs and decreased quality of life. New clinical trial designs such as window-of-opportunity trials will aid in selecting patients that potentially respond to the new drug at an early stage (chapter 2 ). In such cases, the imaging biomarker should accurately predict the drug efficacy firstly to categorize patients. Use of antibodies in a theragnostic approach not only aids in selection of patients but also saves time as diagnosis and therapy occurs simultaneously. In chapter 3 and 4, we used molecular imaging methods for determining antibody uptake in tumors. Antibody uptake in both cases was not directly dependent on the expression levels of the target molecules. In chapter 3, a discrepancy was observed between EGFR expression and cetuximab uptake. Therefore, instead of selecting a patient for cetuximab treatment based on a biopsy determined EGFR status, a positive PET tumor signal upon ${ }^{89} \mathrm{Zr}$-cetuximab imaging is a better alternative. Patients who are EGFR positive but have low ${ }^{89} \mathrm{Zr}$ cetuximab tumor uptake can be categorized for an adjuvant therapy. The main finding of this study is that the PET uptake was associated with microvessel density, one of major barriers for effective delivery of macromolecules. Antibodies in small immuno protein format are therefore attractive alternatives for antibody-based therapy. The small immuno proteins have similar specificity towards the target but have faster clearance properties and thus suitable for both therapy and imaging. In chapter 4, we evaluated the uptake of antibody in a small immuno protein format directed against the catalytic domain of MMP2. The uptake of antibody was dependent on MMP2 activity and not solely on MMP2 expression. Therefore, evaluating MMP2 expression by immunohistochemistry might not provide accurate information about the MMP2 activity and thus selection of right patient for MMP2 targeted treatment is hampered. Since aMMP2-SIP accurately detects MMP2 activity, it might be an attractive theragnostic agent in future. However, additional preclinical studies are required to validate this hypothesis. Since small immuno proteins are superior to intact antibodies in terms of clearance from blood and overcoming barriers for tumor uptake, they are promising as theragnostic agents. Incorporating such agents into window- 
of-opportunity trial designs will accelerate drug development in addition to individualized treatment.

In the second part of thesis, we evaluated the biological basis of radiomics using preclinical studies. Chapter 5 and chapter 6 provide proof-of-concept studies that tumor heterogeneities are indeed reflected by radiomics features. First in chapter 5 , the effect of a genetic change on radiomics features was determined. Significant differences were found in radiomics features which were consistent at an early and late stage post radiation treatment. However, without genetic changes these results were not seen indicating that the genetic change has a direct effect on the image features. This provides a proof-of-concept that genomic changes lead to phenotypic changes, which are reflected by radiomics features. In the next study (Chapter 6), we evaluated if radiomics features can detect the tumoral oxygen status. Oxygen changes not only affected the radiomics features but also the features were associated with hypoxia PET tracer ${ }^{18} \mathrm{~F}-\mathrm{HX} 4$. This study demonstrates that tumor hypoxia can be deciphered by CT derived radiomics features. CT derived radiomics features provide a link between anatomical and molecular information. The predictive power of radiomics features and association to gene signatures provide a promising method for non-radioactive method for determining molecular information. Although the spatial characterization provided by PET imaging cannot be provided by radiomics features, radiomics features from DECT imaging provides an attractive approach for visualizing and quantifying molecular information. Despite the fact that preclinical studies have certain limitation due to small group sizes, they provide an opportunity for determining the effect of a known phenotype on radiomics features and thus help to categorize radiomics features based on biological role. Additional preclinical studies for determining the biological link of radiomics are not only expensive but also time taking but on the other hand, incorporating imaging in preclinical studies pre and post targeted therapy might be an alternative. 


\section{References}

Fass, L. Imaging and cancer: a review. Molecular oncology 2, 115-152 (2008). Gambhir, S. S. Molecular imaging of cancer with positron emission tomography. Nature Reviews Cancer 2, 683-693 (2002).

Weissleder, R. Molecular imaging in cancer. Science 312, 1168-1171 (2006). Lambin, P. et al. Radiomics: Extracting more information from medical images using advanced feature analysis. European Journal of Cancer 48, 441-446, doi: 10.1016/j.ejca.2011.11.036 (2012).

Prescott, J. W. Quantitative Imaging Biomarkers: The Application of Advanced Image Processing and Analysis to Clinical and Preclinical Decision Making. Journal of Digital Imaging 26, 97-108(2013).

Biomarkers Definitions Working, G. Biomarkers and surrogate endpoints: Preferred definitions and conceptual framework. Clinical Pharmacology \& Therapeutics 69, 89-95 (2001).

Peeters, S. G. J. A. et al. TH-302 in Combination with Radiotherapy Enhances the Therapeutic Outcome and Is Associated with Pretreatment [18F]HX4 Hypoxia PET Imaging. Clinical Cancer Research 21, 2984-2992, doi:10.1158/1078-0432.ccr-150018 (2015).

Zegers, C. M. L. et al. In Vivo Quantification of Hypoxic and Metabolic Status of NSCLC Tumors Using [18F]HX4 and [18F]FDG-PET/CT Imaging. Clinical Cancer Research 20, 6389-6397, doi:10.1158/1078-0432.ccr-14-1524 (2014).

Wu, A. M. \& Olafsen, T. Antibodies for molecular imaging of cancer. The Cancer Journal 14, 191-197 (2008).

van Dongen, G. A. M. S., Visser, G. W. M., Lub-de Hooge, M. N., de Vries, E. G. \& Perk, L. R. Immuno-PET: A Navigator in Monoclonal Antibody Development and Applications. The Oncologist 12, 1379-1389 (2007).

Warram, J. et al. Antibody-based imaging strategies for cancer. Cancer and Metastasis Reviews 33, 809-822, doi:10.1007/s10555-014-9505-5 (2014).

van de Watering, F. C. J. et al. Zirconium-89 Labeled Antibodies: A New Tool for Molecular Imaging in Cancer Patients. BioMed Research International 2014, 13, doi:10.1155/2014/203601 (2014).

Verel, I. et al. 89Zr Immuno-PET: Comprehensive Procedures for the Production of 89Zr-Labeled Monoclonal Antibodies. Journal of Nuclear Medicine 44, 12711281 (2003).

4 Deri, M. A., Zeglis, B. M., Francesconi, L. C. \& Lewis, J. S. PET imaging with 89Zr: From radiochemistry to the clinic. Nuclear Medicine and Biology 40, 3-14, doi: 10.1016/j.nucmedbio.2012.08.004 (2013).

5 Zhang, Y., Hong, H. \& Cai, W. PET Tracers Based on Zirconium-89. Current radiopharmaceuticals 4, 131-139 (2011).

6 Tinianow, J. N. et al. Site-specifically 89Zr-labeled monoclonal antibodies for ImmunoPET. Nuclear Medicine and Biology 37, 289-297 (2010).

Nayak, T. K. \& Brechbiel, M. W. Radioimmunoimaging with Longer-Lived Positron-Emitting Radionuclides: Potentials and Challenges. Bioconjugate Chemistry 20, 825-841, doi:10.1021/bc800299f (2009).

18 Holliger, P. \& Hudson, P. J. Engineered antibody fragments and the rise of single domains. Nat Biotech 23, 1126-1136 (2005).

Kaur, S. et al. Recent trends in antibody-based oncologic imaging. Cancer Letters 315, 97-111, doi: 10.1016/j.canlet.2011.10.017 (2012).

Nelson, A. L. Antibody fragments: Hope and hype. mAbs 2, 77-83 (2010).

Brack, S. S., Silacci, M., Birchler, M. \& Neri, D. Tumor-Targeting Properties of Novel Antibodies Specific to the Large Isoform of Tenascin-C. Clinical Cancer Research 12, 3200-3208, doi:10.1158/1078-0432.ccr-05-2804 (2006). 
Smith, S. V. Molecular imaging with copper-64 in the drug discovery and development arena. Expert Opinion on Drug Discovery 2, 659-672, doi:10.1517/17460441.2.5.659 (2007).

Humblet, Y. Cetuximab: an IgG1 monoclonal antibody for the treatment of epidermal growth factor receptor-expressing tumours. Expert Opinion on Pharmacotherapy 5, 1621-1633, doi:10.1517/14656566.5.7.1621 (2004). Wong, S.-F. Cetuximab: An epidermal growth factor receptor monoclonal antibody for the treatment of colorectal cancer. Clinical Therapeutics 27, 684694, doi: 10.1016/j.clinthera.2005.06.003 (2005). Hebbar, M. et al. Lack of usefulness of epidermal growth factor receptor expression determination for cetuximab therapy in patients with colorectal cancer. Anti-cancer drugs 17, 855-857 (2006).

Jain, R. Vascular and interstitial barriers to delivery of therapeutic agents in tumors. Cancer and Metastasis Reviews 9, 253-266, doi:10.1007/bf00046364 (1990).

Arjaans, M. et al. Bevacizumab-induced normalization of blood vessels in tumors hampers antibody uptake. Cancer research 73, 3347-3355 (2013).

Aerts, H. J. W. L. et al. Disparity Between In Vivo EGFR Expression and 89ZrLabeled Cetuximab Uptake Assessed with PET. Journal of Nuclear Medicine 50, 123-131, doi:10.2967/jnumed.108.054312 (2009). Tentler, J. J. et al. Patient-derived tumour xenografts as models for oncology drug development. Nature reviews Clinical oncology 9, 338-350 (2012). Siolas, D. \& Hannon, G. J. Patient-derived tumor xenografts: transforming clinical samples into mouse models. Cancer research 73, 5315-5319 (2013).

1
Liu, Y., Cox, S. R., Morita, T. \& Kourembanas, S. Hypoxia Regulates Vascular Endothelial Growth Factor Gene Expression in Endothelial Cells: Identification of a 5' Enhancer. Circulation Research 77, 638-643, doi:10.1161/01.res.77.3.638 (1995). Krock, B. L., Skuli, N. \& Simon, M. C. Hypoxia-Induced Angiogenesis: Good and Evil. Genes \& Cancer 2, 1117-1133, doi:10.1177/1947601911423654 (2011). Forsythe, J. A. et al. Activation of vascular endothelial growth factor gene transcription by hypoxia-inducible factor 1. Molecular and Cellular Biology 16, 4604-4613, doi:10.1128/mcb.16.9.4604 (1996).

4 Hendriksen, E. M. et al. Angiogenesis, hypoxia and VEGF expression during tumour growth in a human xenograft tumour model. Microvascular Research 77, 96-103, doi: 10.1016/j.mvr.2008.11.002 (2009).

5 Yuan, F. Transvascular drug delivery in solid tumors. Seminars in Radiation Oncology 8, 164-175, doi: 10.1016/S1053-4296(98)80042-8 (1998).

Huang, L. et al. SPECT Imaging with 99mTc-Labeled EGFR-Specific Nanobody for In Vivo Monitoring of EGFR Expression. Molecular Imaging and Biology 10, $167-$ 175, doi:10.1007/s11307-008-0133-8 (2008).

Zhou, Y. et al. Impact of Single-chain Fv Antibody Fragment Affinity on Nanoparticle Targeting of Epidermal Growth Factor Receptor-expressing Tumor Cells. Journal of Molecular Biology 371, 934-947, 10.1016/j.jmb.2007.05.011 (2007).

38 Kohn, E. C. \& Liotta, L. A. Molecular Insights into Cancer Invasion: Strategies for Prevention and Intervention. Cancer Research 55, 1856-1862 (1995).

39 Boissier, S. et al. Bisphosphonates inhibit breast and prostate carcinoma cell invasion, an early event in the formation of bone metastases. Cancer research 60, 2949-2954 (2000).

40 Schmalfeldt, B. et al. Increased Expression of Matrix Metalloproteinases (MMP)2, MMP-9, and the Urokinase-Type Plasminogen Activator Is Associated with 
Progression from Benign to Advanced Ovarian Cancer. Clinical Cancer Research 7, 2396-2404 (2001).

ZHENG, H. et al. Expressions of MMP-2, MMP-9 and VEGF are Closely Linked to Growth, Invasion, Metastasis and Angiogenesis of Gastric Carcinoma. Anticancer Research 26, 3579-3583 (2006).

Brooks, P. C. et al. Localization of matrix metalloproteinase MMP-2 to the surface of invasive cells by interaction with integrin $\alpha v \beta 3$. Cell 85, 683-693 (1996).

Prontera, C., Mariani, B., Rossi, C., Poggi, A. \& Rotilio, D. Inhibition of gelatinase A (MMP-2) by batimastat and captopril reduces tumor growth and lung metastases in mice bearing Lewis lung carcinoma. International journal of cancer 81, 761-766 (1999). glioma cells. PLoS ONE 6, e20614 (2011).

Kargiotis, O. et al. Adenovirus-mediated transfer of siRNA against MMP-2 mRNA results in impaired invasion and tumor-induced angiogenesis, induces apoptosis in vitro and inhibits tumor growth in vivo in glioblastoma. Oncogene 27, 48304840 (2008).

van Duijnhoven, S. M. J., Robillard, M. S., Nicolay, K. \& Grüll, H. Tumor Targeting of MMP-2/9 Activatable Cell-Penetrating Imaging Probes Is Caused by TumorIndependent Activation. Journal of Nuclear Medicine 52, 279-286, doi:10.2967/jnumed.110.082503 (2011).

Parmar, C. et al. Radiomic feature clusters and Prognostic Signatures specific for Lung and Head \&amp; Neck cancer. Scientific Reports 5, 11044, doi:10.1038/srep11044 (2015).

Chicklore, S. et al. Quantifying tumour heterogeneity in 18F-FDG PET/CT imaging by texture analysis. European Journal of Nuclear Medicine and Molecular Imaging 40, 133-140, doi:10.1007/s00259-012-2247-0 (2013).

9 Gillies, R. J., Kinahan, P. E. \& Hricak, H. Radiomics: Images Are More than Pictures, They Are Data. Radiology, 151169, doi:10.1148/radiol.2015151169 (2015).

0 Coroller, T. P. et al. CT-based radiomic signature predicts distant metastasis in lung adenocarcinoma. Radiotherapy and Oncology 114, 345-350, doi: 10.1016/j.radonc.2015.02.015 (2015).

1 Yoon, H. J. et al. Decoding Tumor Phenotypes for ALK, ROS1, and RET Fusions in Lung Adenocarcinoma Using a Radiomics Approach. Medicine 94 (2015).

2 Aerts, H. J. W. L. et al. Decoding tumour phenotype by noninvasive imaging using a quantitative radiomics approach. Nat Commun 5, doi:10.1038/ncomms5006 (2014).

3 Mackin, D. et al. Measuring computed tomography scanner variability of radiomics features. Investigative radiology 50, 757-765 (2015).

Brown, J. M. \& Wilson, W. R. Exploiting tumour hypoxia in cancer treatment. Nat Rev Cancer 4, 437-447 (2004).

5 Vaupel, P. \& Harrison, L. Tumor Hypoxia: Causative Factors, Compensatory Mechanisms, and Cellular Response. The Oncologist 9, 4-9, doi:10.1634/theoncologist.9-90005-4 (2004).

6 Brown, J. M. in Methods in Enzymology Vol. Volume 435 295-321 (Academic Press, 2007).

57 Brizel, D. M., Sibley, G. S., Prosnitz, L. R., Scher, R. L. \& Dewhirst, M. W. Tumor hypoxia adversely affects the prognosis of carcinoma of the head and neck. International Journal of Radiation Oncology* Biology* Physics 38, 285-289 (1997). 
Summary 
Treatment of cancer and monitoring the disease post treatment depends on the complex molecular information obtained both before and after treatment. Molecular imaging for the detection of cancer reflects overall heterogeneity in the tumor quantitatively, longitudinally and noninvasively unlike invasive biopsy. This requires that the imaging tracer/technique should be able to detect the biological process pre and post treatment accurately. Research in medical imaging has been progressing in four main categories 1) improvement of medical equipment (hardware), 2) standardization of protocols worldwide, 3) developing new tracers/imaging biomarkers and 4) advancements in image analysis (software). In this thesis, we focused on the later two innovations. New molecular imaging tracers were evaluated and discussed in the first part of the thesis. In the second part of the thesis we evaluated molecular patterns and tumor heterogeneities on $\mathrm{CT}$ imaging features using Radiomics, an advanced image analysis software for the extraction of imaging features.

\section{Part 1: Evaluation of new antibody based imaging tracers for detecting invasive phenotype}

Antibody-based imaging has a great potential in the area of theranostics because of its high specificity towards the target. Furthermore, theranostics approach where diagnosis is performed with therapeutic compounds, using antibodies aid in faster validation of the antibodies in clinic for both imaging and drug development. Tumors expressing high levels of Epidermal Growth Factor Receptor (EGFR) are associated with a more aggressive phenotype and resistance to treatment. Cetuximab, a monoclonal antibody targeting EGFR has been a standard treatment for colorectal and head and neck cancer patients. However, not all patients screened for EGFR expression has shown to benefit from the treatment. Labeling the antibody with Zirconium-89 $\left({ }^{89} \mathrm{Zr}\right)$ allows for the visualization of the reachability of the therapeutic antibody within the tumor both longitudinally and non-invasively using Positron Emission Tomography (PET). It was shown earlier by our group that $\left[{ }^{89} \mathrm{Zr}\right]$-Cetuximab uptake in tumors did not correlate with EGFR expression. In chapter 3, we evaluated if the antibody uptake is influenced by tumor microenvironmental parameters, such as hypoxia and microvessel density, in three tumor models (SCCNij202, SCCNij3 and SCCNij82), which differ in EGFR expression. We have observed that the uptake of the antibody was independent of EGFR expression and was associated with hypoxia and microvessel density in the tumors. Altogether, the results 
suggest that the tumor uptake of $\left[{ }^{89} \mathrm{Zr}\right.$ ]-Cetuximab was dependent on multiple factors, since both hypoxia and vessel density had significant influence on the uptake of the antibody.

Since, intact antibodies have low penetration capabilities into tumors due to their larger size, small antibody molecules or chimeric antibodies are highly preferred. Small antibodies possess the antigen specificity similar to intact antibody while the smaller size allows for larger reachability into tumor. Matrix metalloproteinase-2 (MMP2) is associated with invasive cancer phenotype and therefore could serve as imaging biomarker for the invasive phenotype. In chapter 4, we evaluated if the small immuno protein antibody of MMP2 (aMMP2-SIP) could be a potential imaging biomarker for imaging MMP2 in three tumor models (HT1080, U373 and U87) and their corresponding MMP2 knock down (MMP2KD) models. These models demonstrated intermediate (U373 and HT1080) to high (U87) MMP2 expression levels. The results clearly demonstrated that the uptake of aMMP2-SIP was dependent on MMP2 activity rather than MMP2 expression. In addition, we have shown that tumor microenvironmental parameters, such as hypoxia, perfusion and vessel density had no influence on the antibody uptake. Since aMMP2-SIP specifically detects MMP2 activity in the tumors without the need for activation unlike other MMP probes, it can be a potential imaging biomarker for imaging MMP2 activity.

\section{Part 2: CT derived radiomics and underlying tumor heterogeneities: proof-of-concept studies}

Computed Tomography imaging is widely used in the clinic mainly for anatomical information. However, it is hypothesized that CT images have hidden information about tumor heterogeneities and molecular patterns that can be unveiled by using advanced image analysis tools such as Radiomics. Radiomics extracts a large number of features based on pixel intensities, distribution of gray level intensities (texture), shape and size. It was indeed clinically proven that the Radiomics signatures derived from different modalities including CT have shown to be prognostic in various cancers and associated with genetic patterns. However, the direct relationship between imaging features and underlying genetic or molecular patterns is not clear. Therefore, in order to establish a direct relationship between genetic patters or tumor heterogeneities and the imaging features, we performed preclinical studies as proof-of-concept in the second part of the thesis. Firstly, in chapter 5, we evaluated if a gene 
change can be detected on CT images using Radiomics. To address this, we used doxycycline inducible GADD34 overexpression in HCT116 tumors were grown as xenografts in mice. Radiomics analysis was performed on CT images (40kVp and $80 \mathrm{kVp}$ ) acquired before radiation treatment at tumor volume of $200 \mathrm{~mm}^{3}$, at 4-day post RT and at tumor volume of $500 \mathrm{~mm}^{3}$ post RT. Consistent features were observed only in gene-induced tumors combined with RT both early and at later time points post. Our results were similar at both energy levels indicating that gene-change could influence image features and a direct relationship exists between gene-change leading to phenotypic changes and imaging features.

We further assessed if tumor heterogeneities such as hypoxia can influence CT imaging features in chapter 6 . We first evaluated if temporary changes in oxygen, such as $7 \%$ oxygen breathing leading to increased hypoxia, can impact the imaging features. Radiomics analysis was performed on CT images acquired before and after oxygen modification. We found 15 features that were significantly different between pre and post $7 \%$ oxygen breathing, common in two independent datasets. We observed a positive correlation with hypoxia, assessed using HX4 PET/CT imaging, for 13 features, while 2 features correlated negatively. We furthermore investigated if imaging features are influenced by hypoxia-targeted treatment, such as the hypoxia-activated prodrug (rats) and nitroglycerin (patients). 6 common features were significantly influenced by these hypoxia directed treatment in rats and patients. All the features correlated with HX4 uptake measured as SUVmean or TBR. One wavelet feature strongly correlated with both hypoxic fraction and hypoxic volume in both datasets. These results suggest that features acquired from $\mathrm{CT}$ imaging are influenced by changes in the levels of hypoxia in the tumor.

In conclusion, this thesis evaluates new imaging tracers and techniques and the influence of tumor microenvironment altogether with a focus to improve cancer detection with higher accuracy and for better treatment monitoring. 


\section{Valorization addendum}


Imaging is a central player for patient selection with the ultimate goal personalizing cancer treatment. Over the last decades, a plethora of new imaging techniques and imaging biomarkers are being developed and reported, demonstrating that imaging is a highly active field of research. The function of an imaging biomarker is the ability to quantitatively measure and also to provide spatial distribution of the target in the tumor. Additionally, imaging biomarkers might also provide and prognostic information. However, the introduction of a new imaging biomarker into clinic practice is not straightforward. Because accumulation of a biomarker also depends on the tumor microenvironment thorough validation using for example correlations with pathology is required. The main aim of this thesis was to evaluate new imaging biomarkers for the detection of aggressive and invasive tumors and to acquire additional information of CT images in the concept of radiomics.

\section{Clinical relevance:}

Cancer is one of the dreadful diseases where the time of detection defines treatment option and outcome. If diagnosed in a too late stage for some cases of advanced cancers, treatment becomes mere palliative. Additionally, administration of drugs that are not effective is not only expensive but also degrades quality of life. With the development of new drugs and moving more and more towards the concept of personalized medicine, accurate selection and follow up of a specific treatment is of prime importance. Similar to the imaging biomarkers, the drug uptake in patients depends on multiple factors and therefore a simple biopsy might not be able to reflect the distribution of drug and the clinical outcome. Imaging thus can aid in patient selection and in monitoring treatment responses quantitatively as well as the spatial localization of the target molecule. Imaging can also provide prognostic information of the patient and therefore, can be beneficial to the clinician in making decisions. However, the use of the correct imaging technique is necessary and the imaging information should be reliable. Therefore, validation of an imaging technique/ tracer is required which means standardization of a procedure, the characterization of an imaging biomarker, overcoming the challenges with tumoral uptake and minimizing the background uptake. For this purpose, imaging has been an active field of research and numerous research studies are being conducted. Complementary information on tracer characteristics, time of imaging, influence of tumor microenvironment etc. (chapter 2, chapter 3 and chapter 4) can be attained by performing preclinical studies which aids in standardization of protocols in the clinic. For example, many new hypoxia tracers have been 
developed such as FAZA, FMISO and HX4. However comparative studies in a same tumor model helps to characterize the tracers and to choose the correct tracer (chapter 2). In addition, choice of right tracer for a specific process is necessary.

Several FDA approved antibodies are currently used for the treatment of various cancers e.g. Bevacizumab (target VEGF), Cetuximab (target EGFR) and Trastuzumab (target HER2). However, not all patients screened for the presence of the target benefit from the treatment. For example, cetuximab has been a standard treatment for colorectal and head and neck cancer patients, it was reported that prior selection of patients based on EGFR expression was not beneficial. Preclinical studies using $\left[{ }^{89} \mathrm{Zr}\right.$ ]-Cetuximab imaging in tumor models expressing different EGFR expression have verified that indeed a disparity exits between the EGFR expression and Cetuximab uptake. This disparity could be due to the influence of tumor microenvironmental parameters such as vessel density, hypoxia and necrosis (chapter 3 ). Thus, preclinical studies aid to validate findings and strengthen the information regarding drug development. ${ }^{89} \mathrm{Zr}$ ]-Cetuximab imaging which is already in clinical trials has shown good correlations between the imaging and treatment responses. Hence, antibody based imaging accelerates development of therapeutic antibodies since these antibodies are already well characterized and the imaging information provides the actual distribution of the antibody in the tumors and hence can be used for theranostic purposes.

Small antibody fragments or chimeric antibodies have better reachability in the tumors due to their smaller size. In this thesis, we evaluated if a new antibody, which is produced in small immune protein format against MMP2 can be used for imaging purposes (Chapter 4). Several broadspectrum inhibitors including Bisphosphonates, chemically modified tetracycline derivatives (e.g. col-3), hydroxomates (batimastat and its orally administrative analogue marimastat) have shown to be potent inhibitors of metastases and tumor growth in preclinical studies. However, the clinical trials were disappointing. The lack of knowledge on specificity of these broad-spectrum inhibitors and complexity of the nature of MMPs has been attributed to the failure of these inhibitors. Furthermore, patients with early stage cancers were excluded in clinical trials with broad-spectrum inhibitors while MMPs were also reported to play critical role in early stages of cancer development. Therefore, selection of patients based on presence of specific MMP activity is necessary. Furthermore, MMP2 activity is localized in the invasive front and therefore imaging using MMP2 antibody might help in identifying ${ }_{145}$ 
intrusive tumor areas before spreading metastases. Although further research is warranted with aMMP2-SIP as an imaging biomarker, the accurate detection of MMP2 activity despite the decreased levels of MMP2 protein makes it a promising tool for imaging MMP2. In the second part of the thesis, CT imaging has been used to understand the molecular patterns within tumors. CT imaging is widely used in clinic and is less expensive than PET imaging. Imaging features extracted from CT images using Radiomics, an advanced image analysis platform, has been proven to be prognostic in patients. However, little is known about the direct relationship between these image features and gene patterns. Therefore, the research conducted in second part of the thesis is to evaluate further in-depth to understand whether tumor heterogeneities and molecular patterns influence image features. Once, the direct relationship is established, these CT imaging features can deliver both anatomical and molecular information.

\section{Road to the market:}

Imaging with therapeutic antibodies has the benefit of faster translation to clinic, as the antibodies are prior validated for safety and biodistribution in therapeutic settings. However, tagging the antibody can sometimes change the properties of the antibody. Therefore preclinically and clinically the safety profile needs to be tested. $\left[{ }^{89} \mathrm{Zr}\right]$-Cetuximab imaging has shown improved therapeutic results. Validation of the tracer including parameters that can influence an uptake can give additional information for the clinician. New antibodies take lot more time for translation into clinic as several steps such as timing of imaging and tracer characteristics needs to be optimized like. aMMP2-SIP is directed towards active MMP2 and is proven to detect MMP2 activity even when MMP2 protein levels are reduced. However, the main goal is to validate the antibody as an invasive biomarker. Therefore, further research is warranted using orthotopic models to detect the invasive front. On the other hand, CT imaging is widely used in clinic to detect cancer. Therefore, the application of radiomics in clinical settings is easier. However, strong external validations are required when extrapolating the imaging features from preclinical to clinical cases, as the patient tumors are more complicated. More relevant models such as orthotopic or spontaneous models could be used for better translation into clinic. 


\section{Societal Benefit:}

Detection of cancerous tissue accurately saves time and treatment costs for the patient. For instance, after a surgical tumor resection, the specimen needs to be tested for any intrusive tumor cells. If there is any invasive tumor tissue, a second surgery might be needed. Under the guidance of proper imaging tool (e.g. MMP2 imaging) invasive areas of tumor could be detected noninvasively and hence cost-effectiveness will be improved. During and post treatment using this theranostic approach helps to avoid unnecessary treatments. If a patient has high EGFR expression but low Cetuximab uptake, he/she will not benefit from Cetuximab treatment. Prior selection and follow up using $\mathrm{Zr}^{89}$-Cetuximab imaging can help not only the patient but also the doctor in making right decisions. In most of the cancer centers, PET/CT imaging is performed. While PET gives molecular information and CT gives anatomical information, deriving imaging features from both using Radiomics gives additional information for better treatment. This adds valuable information to the doctor and no additional costs to the patient. 
Acknowledgements 
మాత్రు దేవో భవ (Maatru devo bhava)

పిత్రు దేవో భవ (Pitru devo bhava )

ఆచర్య దేవో భవ (Aacharya devo bhava)

Meaning: Revere mother as God, father as God and teacher as God

To my Family:

\begin{abstract}
ముందుగా నా తలిదండ్రులకు నా క్రుతజ్ఞుతలు. వారు నా ఉన్నతే ద్యేయంగా పెట్టుకుని, అన్ని వ్యయ ప్రయాసలకు ఓర్చి, నన్ను ఇంత దాన్నిగా తోర్చిదిద్దారు. వారు నామిద పెట్టుకున్న ఆశలు వమ్ము చెయలేదనే అనుకుంటాను. నాన్న పైచదువులు చదవలేదనె లోటు నా ద్వారా త్రేర్చుకున్నారు అనుకుంటా. అమ్మా నువ్వు అహర్నిశమూ నన్ను అంటిపెట్టుకుని ఉండి నన్ను ముందుకు నడిపించిన నికు ఏమిన్వగలను?

మెట్టినింట్లో నాకు రెండవ తలిడండ్రులు దొరకడం నా అద్రుష్టం. నేను నా పే హెచ్ ఉ చేస్తున్న సమయంలో అన్ని రకాల ప్రోత్సాహం ఇచ్చినందుకు నా హ్రుదయపూర్వక క్రుతజ్ఞాలలు.
\end{abstract}

Raviii, my sweetheart you have given me everything you could and stood by me through thick and thin. Cannot forget the days when we had to struggle through the distance apart but you were the one who instilled strength, courage and motivation. Cannot forget the days when it was extremely difficult to say goodbye and to wait until the next meet. But all I remember is the intense love and trust we share. Life is so beautiful with you. Love you more than anything else. You are a perfect son, perfect son-in-law, a perfect husband and I know you will be a perfect dad too :)

Srikant, Sowmya, Uma akka, jiju thank you for the support. Sowmya thank you for all the care you have given to the family and for all the little gifts you have sent.

\title{
To people in MaastRO:
}

This PhD would not have been possible without the contribution of many people. I express my sincere gratitude to all those who have contributed to my PhD directly and also people who have indirectly helped me by giving their moral support

Philippe, I am very thankful to you for giving me the opportunity to do my $\mathrm{PhD}$ at MaastRO. In the beginning I was hesitant to approach you but when I spoke to you, you boosted my confidence. I will never forget the encouraging words you have spoken to me at meetings and in person. You have discovered potential in me which I never realized I possessed. Thanks for the trust you placed in me. 
Ludwig, I don't know where to start from and how to thank you. Voila!!! You are the 'Mr. cool'. You always had lot of patience to listen and at times I wondered if you ever could get angry. You were always approachable and very friendly. Thank you for all the encouragement and support you have given through out my PhD. You have always given me the freedom to explore and thanks to all the weekly discussions we had. I have learnt a lot from you all these years. You are a very positive person and this definitely had a great impact on me. Big big thanks for all the hardwork you did to push my projects.

Special thanks to my thesis assessment committee: Prof. Dr. F.C.S Ramaekers, Prof. Dr. Dario Neri, Prof. Dr. F. Mottaghy, Prof. Dr. Andre Dekker and Dr. Hugo Aerts for their time, consideration and valuable remarks.

Thanks to all co-authors for their scientific input and contributions.

Marc, you have always been very kind to me. As the head of the department you made sure that the international people in the lab were welcomed. You have always encouraged me. Thank you for all the discussions and for all the practical counseling.

Twan, I cannot thank you enough for taking out special time for teaching and training me. You are very kind person and always been very supportive and instilled a lot of confidence in me. I have learnt a lot from you especially when doing invitro experiments. I am really grateful to you for all the time and help you have extended.

To all the people who were part of Maatro lab during my PhD: Marc, Arjan, Jan, Kasper, Twan, Carla, Barry, Hanneke, Tom, Roger, Carolien Wansleeben, Kim P, Eloy, Lorena, Kim S, Sarah, Marike, Marco, Linda, Venus, Sanaz, Ludwig, Ala, Ruchi, Daniela, Natasja, Rianne, Linda, Nicole, Simon, Raymon, Paolo, Tessa, An, Frank, Caroline Hodin, Alexey, Milou, Dana, Judith, Maria a big thank you to each one of you. You have been wonderful colleagues and friends. Thank you for all the discussions, for the coffee and vlaai breaks, for the friendly atmosphere. I had great time!

Thanks to all the people from mCAT group: Philippe, Ludwig, Ala, Maud, Sarah, Marike, Simon, Raymon, Nicole, Linda, Natasja, Rianne, An thank you for your inputs. Veronica, I had less interaction with you, but I hope you are enjoying your $\mathrm{PhD}$ and I wish you all the best! 
Ala, Nicole, Simon my roomies!!!! Thank you for all the special moments we shared. Each one of you has special qualities that I always felt that I could learn. Ala, you are very talented and have an amazing understanding of varied subjects. You have been very helpful and gave great ideas. You never have fear for anything and you are a very confident person, which is greatest quality that I always would want to learn. Thank you for being part of my thesis and for thinking along for my projects.

Nicoleeeeee, thank you for the energetic and fun filled moments you brought into the room. We had lot of light moments together. You have great positive energy that is very infectious. You always knew what you wanted and you knew how to get it too. You have very clear thoughts.

Simon, you are always cool and very organized person. I had great time with you and talking to you has always been fun. All the very best for finishing up your PhD.

Arie, Ruchi and Marike, you were the first friends I made in the lab. Arie you are one of special kind. Thanks to all those lively moments when Nicole, Marco, You and I were roomies. I will never forget your Tuesday offs and the funny conversations. You are very enthusiastic about your work, a great quality to learn. You are very kind and I cannot forget the day when I was missing my family and you comforted me, invited me for dinner. Jenny is a wonderful woman and you both will always remain special to me.

Ruchi, you made me feel comfortable in the lab when I initially arrived. I will never forget your advices and the dabba you packed for me :). Thank you for all the care you have shown during the short time.

Marike we joined almost at same time. We were in same boat giving some kind of consolation to each other when experiments failed. But we kept our determination and aim. You are about to finish soon too. Keep up the good work. You are almost there!!! Whoop Whoop!! Thank you for the energy cookies and motivating words you gave me during the holidays.

Natasja, Rianne without both of your help my PhD would have taken longer I guess. You both have been very helpful throughout my PhD. Natasja, you are such a superb animal technician. Thanks to all the pep talks and the tea moments we shared. And thank you for keeping in touch with me after I left the lab and knowing about my whereabouts. Rianne, you are a sweet girl, very energetic, very bright and always cheerful. You always made an impression that any work is easy for you. Thanks for all the help darling. 
Kasper, Maud Thanks a lot for your support! You always extended help when needed.

Jan, you were very kind to me from the beginning. I will never forget your help and encouraging words and advices.

Carla, special thanks to you for taking care of the orders, administrative things and for timely help. You were very patient always when I came to ask something.

Kimmy S you are very cheerful person and I love the way you sing in the lab that always brought a smile.

Sarah, Marco, Eloy, you are very nice people. thank you for your friendship and fun time. Sarah, thanks a lot for all the quick responses to my emails and discussions.

Lydie, my little girl I really enjoyed going to Zumba with you. Thank you for all the nice weekends.

Sanazyy, sweetie you were very kind to me and never said no when I asked for help despite being extremely busy. Thank you for taking time out during weekends to make me feel better. You have always been a good listener and very genuine friend. I love the time spent with you.

Venus, you are such a darling!! You are one of those friends whom I would want to be friends with forever. Thanks to all those pampering dinners and extra care you have shown. Those weekend lazy and cozy evenings till midnight will never be forgotten. You and I have a great understanding and you always made me feel comfortable. Thank you for everything!

People from Maastro clinic: Sara, Ralph, Rianne Herben, Karen, Wouter, Stefan, Aniek, Ruben, Evelyn, Patrick, Frank Hoebers, Andre, Maaike, Sean, Jonathan, Skadi, John Paulissen, Ellen, Erik, Cary, Timo, Maaike, Marlies, Jurgen, Shane, Ruud, Lotte, Floor. Thank you all. You have been wonderful colleagues.

Sara and Ralph, thank you for all the help. Ralphie, the radiomics guy thank you for the help with radiomics and trying to explain all the complicated codes:)

Sara, thank you so much for all the help. You made my life easier by giving simpler explanations :D. We had a great understanding at work. You are very lively person. Thanks again for taking time out for helping me. Good 
luck for your defense. I am sure you will do great!

Karen, I adore you so much. You are one kind of person that everyone would want to be with. Talking to you calms down and puts the other person to peace. You are highly talented yet very humble person, which makes you very special.

Ellen, thank you for all the help. It was always easy to talk to you and you were very helpful in arranging our visas and other administrative things.

Patrick, Stefan, thanks a lot for the help with the Ssmart. I had to bother you many times with the machine problems but you were always nice and friendly.

Frank Verhaegen, Wouter and Frank Hoebers thank you for your scientific input and contributions

People of CPV: Richard, Saskia, Rick, Clarice and people of RNL: Martine, Sandra thank you all for your efforts and help

Jos Broers from department of Molecular and Cellular Biology and Mario Losen from Department of Neuroscience (MHeNS), thank you for your guidance.

I would like to specially thank Prof. Dr. Dario Neri and Marcel Weber from ETH Zurich for providing with the aMMP2-SIP antibody.

People outside MaastRO but part of maastricht family: Ajay, Ruhi, Jelly, Sridhar, Prabha, Ramesh, Swati, Shilpi, Subhashis, Behnaz, Rio a big thank you to all of you.

Dearest Ajay! Thanks a lot for all the care and love you have shown towards us. I will never forget your care for me when I was sick and the delicious dinners you prepared. You made the weekends much more positive. Thanks a lot!

People outside Maastricht have also given their constant support and encouragement: Dr. Laurent Besret, Krishna, Ravi Rangara, Bhuvana akka, Prasu, Prof. Dr. A. Sadanandam garu and Dr. Navin Twarakavi thanks a lot for everything. Navin annayya thank you for taking time out to design the cover page and for all the motivating words. 
Curriculum Vitae 
Kranthi Panth born on November $1^{\text {st }} 1986$ in Hyderabad, Telangana state, India spent all her childhood and did schooling in Warangal, Telangana state India. She completed M.Sc in Biotechnology from Kakatiya University, Warangal in 2009. During this time she also did summer internship from Centre for Cellular and Molecular Biology, Hyderabad gaining experience in Protein Biochemistry. Having interest in Molecular Imaging, she joined European Masters in Molecular Imaging (EMMI) in Saclay, France in 2009. During her European Masters, she did summer internships first at Prof. Dr. Bertrand Tavitian's lab at Saclay, France and then Prof. Dr. Andreas Jacobs' lab at Munster, Germany in 2010 gaining experience in Optical Imaging techniques. In 2011, she did her senior internship at Molecular Imaging facility of Sanofi Oncology under the guidance of Dr. Chantal Carrez and Dr. Laurent Besret. At Sanofi, she worked on validation of $\left[{ }^{18} \mathrm{~F}\right]$-FLT PET with patient derived tumor models. Kranthi started her PhD in January 2012 at Maastricht University, department of radiation oncology (MaastRO lab) under the supervision of Prof. Dr. Phillipe Lambin \& Dr. Ludwig Dubois. Her research at Maastricht focused on developing new imaging methods involving various modalities. The results of her research are presented in this thesis. 


\section{Publications}


L. J. Dubois, R. Niemans, S. J. A. van Kuijk, K. M. Panth, N. K. Parvathaneni, S. G. J. A. Peeters, C. M. L. Zegers, N. H. Rekers, M. W. van Gisbergen, R. Biemans, N. G. Lieuwes, L. Spiegelberg, A. Yaromina, J. Y. Winum, M. Vooijs and P. Lambin. New ways to image and target tumour hypoxia and its molecular responses. Radiotherapy and Oncology 116(3): 352-357.

$>\quad$ K. M. Panth, R. T. H. Leijenaar, S. Carvalho, N. G. Lieuwes, A. Yaromina, L. Dubois and P. Lambin. Is there a causal relationship between genetic changes and radiomics-based image features? An in vivo preclinical experiment with doxycycline inducible GADD34 tumor cells. Radiotherapy and Oncology. 116: 462-6.

$>$ P. Lambin, J. Zindler, B. G. L. Vanneste, L. V. De Voorde, D. Eekers, I. Compter, K. M. Panth, J. Peerlings, R. T. H. M. Larue, T. M. Deist, A. Jochems, T. Lustberg, J. van Soest, E. E. C. de Jong, A. J. G. Even, B. Reymen, N. Rekers, M. van Gisbergen, E. Roelofs, S. Carvalho, R. T. H. Leijenaar, C. M. L. Zegers, M. Jacobs, J. van Timmeren, P. Brouwers, J. A. Lal, L. Dubois, A. Yaromina, E. J. Van Limbergen, M. Berbee, W. J. van Elmpt, C. Oberije, B. Ramaekers, A. Dekker, L. J. Boersma, F. Hoebers, K. M. Smits, A. J. Berlanga and S. Walsh. Decision Support Systems for Personalized and participative Radiation Oncology. Advanced Drug Delivery Reviews. In Press

$>\quad$ K. M. Panth, T. van den Beucken, R. Biemans, N. G. Lieuwes, M. Weber, M. Losen, A. Yaromina, L. J. Dubois and P. Lambin. In vivo optical imaging of MMP2 immuno protein antibody: tumor uptake is associated with MMP2 activity. Scientific Reports 6, 22198.

$>$ R.T.H.M Larue, L. Van De Voorde, M. Berbée, W. J. Van Elmpt, L. J. Dubois, K. M. Panth, S.G.J.A Peeters, A. Cleassens, W.M.J Schreurs, M. Nap, F.A.R.M Warmerdam; F. Erdkamp; M.N. Sosef; P. Lambin. A phase 1 "window" trial testing evofosfamide (TH-302), a tumour-selective hypoxia-activated cytotoxic prodrug, in combination with preoperative chemoradiotherapy in patients with oesophageal adenocarcinoma. Submitted to BMC cancer

$>\quad$ K. M. Panth, S.G.J.A Peeters, N. G. Lieuwes, A. Yaromina, A. J. Poot, J. Bussink, G.U.U.S van Dongen, L.J. Dubois and P. Lambin. $\left[{ }^{89} \mathrm{Zr}\right]-$ Cetuximab uptake in primary head and neck xenografts depends on tumor micro-environmental characteristics. Manuscript in preparation.

$>\quad$ K. M. Panth, S. Carvalho, R.T.H. Leijenaar, S.G.J.A Peeters, A. J. G. Even, C. M. L. Zegers, B Reymen, E. Van Elmpt, A. Yaromina, L. J. Dubois, P. Lambin. Do CT derived radiomics features define oxygenation status of tumors? Manuscript in preparation.

$>$ P. Lambin, R.T.H. Leijenaar, J. Peerlings, J. van Soest, E. de Jong, J. van Timmeren, A. J. G. Even, R. Larue, T. Deist, A. Jochems, Y. van Wijk, T. Lustberg, K. M. Panth, N. Rekers, W. J. van Elmpt, J. Wildberger, F. Mottaghy, A. Dekker, and S. Walsh. Radiomics: the bridge between medical imaging and personalized medicine. Manuscript in preparation. 


\section{Conferences:}

$>$ Selected talk, Do radiomics features excel human eye in identifying an irradiated tumor? Rat tumor to patient HNSCC, ESTRO 35 Turin, Italy, April 2016

Selected talk, In vivo optical imaging of MMP2 immuno protein antibody: the uptake in xenograft tumors is associated with MMP2 activity, GROW science day, Maastricht, November 2015

> Selected talk, In vivo optical imaging of MMP2 immuno protein antibody, New Advances in animal Models and preclinical Imaging for translational Research in Cancerology, Nantes, France, September 2015

$>$ Selected talk, Is there a causal relationship between genetic changes and radiomics-based image features? European Molecular Imaging Meeting, Tubingen, Germany, March 2015

> Selected poster presentation, In vivo optical imaging of MMP2 immuno protein antibody European Molecular Imaging Meeting, March 2015

$>$ Selected poster presentation, $\left[{ }^{89} \mathrm{Zr}\right]$ - Cetuximab uptake in primary head and neck xenografts is independent of the expression of EGFR, ESTRO 33, Vienna, Austria April 2014

Awards:

Active participation award and travel award, New Advances in animal Models and preclinical Imaging for translational Research in Cancerology, Nantes, France, September 2015

$>$ Best Poster award in the category: Visualization of tumour and the microenvironment, European Molecular Imaging Meeting, Tubingen, Germany, March 2015

Nominated for young investigator award, European Molecular Imaging Meeting, Tubingen, Germany, March 2015 


\section{"Dedicated to the bravehearts who fight cancer with drive and courage}

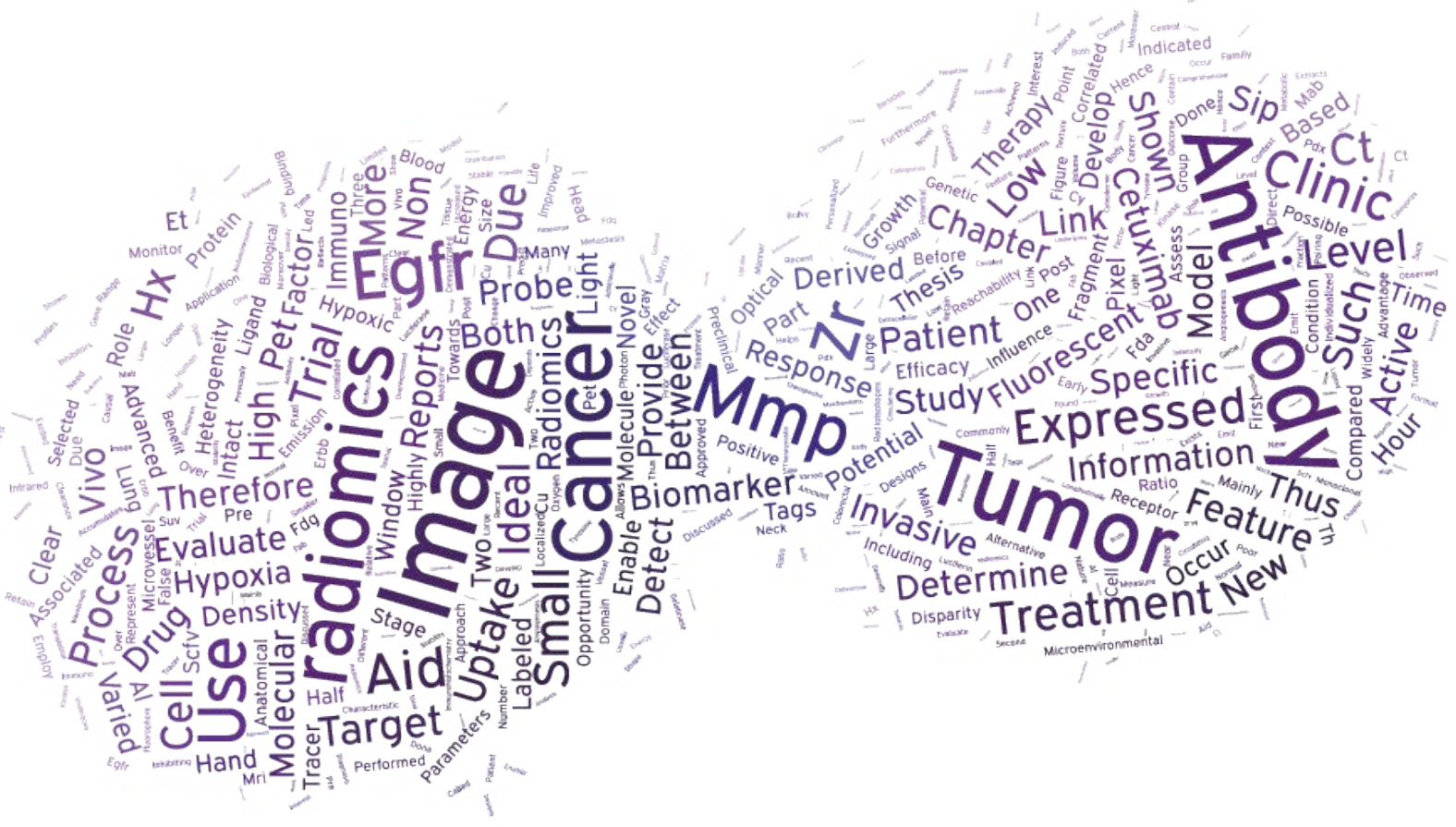

